\title{
On the Fourier-Jacobi expansion of the unitary Kudla lift
}

\author{
Atsushi Murase and Takashi Sugano
}

\begin{abstract}
We study a theta lift from a cusp form $f$ on $U(1,1)$ to a cusp form $\mathcal{L} f$ on $U(2,1)$ (the unitary Kudla lift of $f$ ). We give an explicit expression of the Fourier-Jacobi expansion of $\mathcal{L} f$ in terms of periods and Hecke eigenvalues of $f$. As an application, we give a criterion for the nonvanishing of $\mathcal{L} f$.
\end{abstract}

\section{Introduction}

1.1 Let $f$ be a holomorphic cusp form on $U(1,1)$. In [Kud81], Kudla constructed a holomorphic cusp form $\mathcal{L} f$ on $U(2,1)$ by integrating $f$ against a theta kernel on $U(2,1) \times U(1,1)$ (see also $[$ Kud79]). Furthermore, he showed that $\mathcal{L} f$ is a Hecke eigenform if so is $f$, and gave a relation between an automorphic $L$-function of $\mathcal{L} f$ and that of $f$. The main object of the paper is to study the Fourier-Jacobi expansion of $\mathcal{L} f$. In particular, we show that the primitive components of $\mathcal{L} f$ are expressed in terms of certain $U(1)$-periods of $f$, and that nonprimitive components of $\mathcal{L} f$ satisfy certain recursion relations if $f$ is a Hecke eigenform. As an application of these results, we obtain a criterion for the nonvanishing of $\mathcal{L} f$ (for a nonvanishing criterion in a representation theoretic setting, we refer to [GRS97] and [Tan99]; see the remark after Main Theorem III in $§ 1.4)$.

1.2 To be more precise, let $K$ be an imaginary quadratic field of discriminant $D$. Let $G=U(2,1)=$ $U(S)$ and $H=U(1,1)=U(T)$, where

$$
S=\left[\begin{array}{lll} 
& 1^{1 / \sqrt{D}} \\
-1 / \sqrt{D} &
\end{array}\right], \quad T=\left(\begin{array}{rr} 
& 1 \\
-1 &
\end{array}\right) .
$$

As is well known, $(G, H)$ forms a dual reductive pair. Let $\mathcal{X}$ be the set of Hecke characters $\chi$ of $K$ satisfying $\left.\chi\right|_{\mathbb{Q}_{\mathbf{A}}^{\times}}=\omega$, where $\omega$ denotes the quadratic Hecke character of $\mathbb{Q}$ corresponding to $K / \mathbb{Q}$. For $\chi \in \mathcal{X}$, let $w_{\infty}(\chi)$ be the integer such that $\chi\left(z_{\infty}\right)=\left(z_{\infty} /\left|z_{\infty}\right|\right)^{w_{\infty}}(\chi)$ for $z_{\infty} \in K_{\infty}^{\times}=\mathbb{C}^{\times}$. Fix a positive integer $l$ divisible by $w(K)$, the number of roots of unity in $K$. We also fix a character $\chi_{0} \in \mathcal{X}$ with $w_{\infty}\left(\chi_{0}\right)=-1$. The character $\chi_{0}$ determines a splitting $\mathcal{M}_{\chi_{0}}$ of metaplectic representations of $G_{\mathbf{A}} \times H_{\mathbf{A}}$ on $\mathcal{S}\left(K_{\mathbf{A}}^{2}\right) \otimes \mathcal{S}\left(K_{\mathbf{A}}\right)$, where $\mathcal{S}\left(K_{\mathbf{A}}^{m}\right)$ denotes the space of Schwartz-Bruhat functions on $K_{\mathbf{A}}^{m}$. We then construct a theta kernel

$$
\theta(g, h)=\chi_{0}^{-1}(\operatorname{det} g) \chi_{0}^{-2}(\operatorname{det} h) \sum_{X \in K^{2}, \xi \in K} \mathcal{M}_{\chi_{0}}(g \times h)\left(\Phi_{0} \otimes \varphi_{0}\right)(X, \xi)
$$

Received 26 March 2005, accepted in final form 16 June 2006.

2000 Mathematics Subject Classification 11F55 (primary), 11F67, 11F70 (secondary).

Keywords: automorphic forms, Fourier-Jacobi expansion, theta lift, Kudla lift, unitary groups.

This journal is (C) Foundation Compositio Mathematica 2007. 


\section{A. Murase And T. Sugano}

with a suitably chosen test function $\Phi_{0} \otimes \varphi_{0} \in \mathcal{S}\left(K_{\mathbf{A}}^{2}\right) \otimes \mathcal{S}\left(K_{\mathbf{A}}\right)$ depending on $l$. Let $f$ be a holomorphic cusp form on $H_{\mathbf{A}}$ of weight $l-1$, level $D$ and character $\tilde{\chi}_{0}$ (cf. $\S 3.3$ ). Set

$$
\mathcal{L} f(g)=\int_{H_{\mathbb{Q}} \backslash H_{\mathbf{A}}} f(h) \theta(g, h) d h \quad\left(g \in G_{\mathbf{A}}\right) .
$$

The theta lift $\mathcal{L} f$ was first studied by Kudla and we call $\mathcal{L} f$ the unitary Kudla lift of $f$. Kudla showed the following [Kud79, Kud81]:

(1.1) $\mathcal{L} f$ is a holomorphic cusp form on $G_{\mathbf{A}}$ of weight $l$ and level 1 ;

(1.2) if $f$ is a Hecke eigenform, so is $\mathcal{L} f$.

He also gave a relation between an automorphic $L$-function of $\mathcal{L} f$ and that of $f$.

1.3 In [Shi79], Shintani investigated the theory of (refined) Fourier-Jacobi expansions of automorphic forms on $G$. It should be noted that Piatetski-Shapiro independently developed a similar theory from a more representation theoretic point of view (see [Pia80] and [BPR03]). In this subsection, we briefly recall Shintani's theory. For details, see $\S \S 4$ and 5 . Let $R$ be an algebraic subgroup of $G$ given by

$$
R_{\mathbb{Q}}=\left\{(w, x) t:=\left[\begin{array}{ccc}
1 & \sqrt{D} w^{\sigma} & x+\sqrt{D} w w^{\sigma} / 2 \\
0 & 1 & w \\
0 & 0 & 1
\end{array}\right]\left[\begin{array}{ccc}
1 & & \\
& t & \\
& & 1
\end{array}\right] \mid w \in K, x \in \mathbb{Q}, t \in K^{1}\right\},
$$

where $\sigma$ stands for the nontrivial automorphism of $K / \mathbb{Q}$ and $K^{1}=\left\{t \in K^{\times} \mid t t^{\sigma}=1\right\}$. Denote by $\psi$ the additive character of $\mathbb{Q}_{\mathbf{A}} / \mathbb{Q}$ determined by $\psi\left(x_{\infty}\right)=\exp \left(2 \pi \sqrt{-1} x_{\infty}\right)\left(x_{\infty} \in \mathbb{R}\right)$. Let $F$ be a holomorphic cusp form on $G_{\mathbf{A}}$ of weight $l$ on $\mathcal{K}_{f}=\prod_{p<\infty} G\left(\mathbb{Z}_{p}\right)$ (cf. $\S 3.1$ ). Then $F$ admits the Fourier-Jacobi expansion

$$
F(g)=\sum_{m \in \mathbb{Q}, m>0} F^{m}(g) \quad\left(g \in G_{\mathbf{A}}\right)
$$

where

$$
F^{m}(g)=\int_{\mathbb{Q}_{\mathbb{Q}} \mathbf{A}} \psi(-m x) F((0, x) g) d x
$$

Let $\mathfrak{a}$ be a nonzero fractional ideal of $K$ and take an element $\alpha_{f}$ of $K_{\mathbf{A}, f}^{\times}$(the finite part of $K_{\mathbf{A}}^{\times}$) corresponding to $\mathfrak{a}$ (cf. $\left.\S 2.2\right)$. Let $F_{\mathfrak{a}}^{m}$ be the function on $R_{\mathbb{Q}} \backslash R_{\mathbf{A}}$ given by $F_{\mathfrak{a}}^{m}(r)=F^{m}(r$. $\left.\operatorname{diag}\left(\alpha_{f}^{\sigma}, 1, \alpha_{f}^{-1}\right)\right)\left(r \in R_{\mathbf{A}}\right)$. Let $\chi \in \mathcal{X}$ with $w_{\infty}(\chi)=-1$ and suppose that $\chi$ satisfies certain conditions on its Artin conductor and epsilon factor. Then there exists a nonzero theta function $\Theta_{\mathfrak{a}, \chi}^{m}: R_{\mathbb{Q}} \backslash R_{\mathbf{A}} \rightarrow \mathbb{C}$, which is primitive and an eigenfunction with respect to the action of $U(1)$ with eigenvalues determined by $\chi$ (for a more precise statement, see $\S 4.6$ ). It is known that $\Theta_{\mathfrak{a}, \chi}^{m}$ is unique up to constant multiples (see Theorem 4.4). For a fractional ideal $\mathfrak{b}$ of $K$ with $\mathfrak{b} \subset \mathfrak{a}$, put

$$
C_{F}\left(\mathfrak{b} ; \Theta_{\mathfrak{a}, \chi}^{m}\right):=\int_{R_{\mathbb{Q}} \backslash R_{\mathbf{A}}} F_{\mathfrak{b}}^{m}(r) \overline{\Theta_{\mathfrak{a}, \chi}^{m}(r)} d r .
$$

We call $\left\{C_{F}\left(\mathfrak{b} ; \Theta_{\mathfrak{a}, \chi}^{m}\right) \mid m, \mathfrak{a}, \mathfrak{b}, \chi\right\}$ the Fourier-Jacobi components of $F$. If $\mathfrak{b}=\mathfrak{a}$, the Fourier-Jacobi component $C_{F}\left(\mathfrak{a} ; \Theta_{\mathfrak{a}, \chi}^{m}\right)$ is said to be primitive. In his pioneering work [Shi79], Shintani showed the following results (cf. $\S 5$ ):

(1.3) $F$ is completely determined by its Fourier-Jacobi components;

(1.4) $F$ is a Hecke eigenform if and only if, for each $(m, \mathfrak{a}, \chi),\left\{C_{F}\left(\mathfrak{b} ; \Theta_{\mathfrak{a}, \chi}^{m}\right) \mid \mathfrak{b} \subset \mathfrak{a}\right\}$ satisfies certain recursion relations;

(1.5) assume that $F$ is a Hecke eigenform, then $F \neq 0$ if and only if at least one of the primitive components of $F$ is nonzero. 


\section{ON THE Fourier-JACOBI EXPANSION OF THE UNITARY KUDLA LIFT}

1.4 Let $\mathcal{L} f$ be the unitary Kudla lift of a holomorphic cusp form $f$ on $H_{\mathbf{A}}$ of weight $l-1$, level $D$ and character $\widetilde{\chi}_{0}$. Let $(m, \mathfrak{a}, \chi)$ be as in $\S 1.3$. Set

$$
W_{\chi, f}^{m}(h)=\int_{K^{1} \backslash K_{\mathbf{A}}^{1}}\left(\chi / \chi_{0}\right)^{1}\left(t^{-1}\right) f\left(\iota_{m}(t) h\right) d^{\times} t \quad\left(h \in H_{\mathbf{A}}\right),
$$

where $\iota_{m}$ is an embedding of $K^{1}$ into $H$ depending on $m$ defined by (7.6) (for the definition of $\left(\chi / \chi_{0}\right)^{1}$, see $\left.\S 2.5\right)$. The function $W_{\chi, f}^{m}$ on $H_{\mathbf{A}}$ can be seen as a spherical function for $(U(1,1), U(1))$. We now state the main results of the paper.

MaIn Theorem I (Theorem 7.5). The primitive Fourier-Jacobi components of $\mathcal{L} f$ are given as follows:

$$
C_{\mathcal{L} f}\left(\mathfrak{a} ; \Theta_{\mathfrak{a}, \chi}^{m}\right)=c(m, \mathfrak{a}, \chi) \cdot \overline{I\left(\Theta_{\mathfrak{a}, \chi}^{m}\right)} \cdot J(m, \mathfrak{a}, \chi, f),
$$

where $c(m, \mathfrak{a}, \chi)$ is a nonzero explicit constant depending only on $(m, \mathfrak{a}, \chi)$,

$$
I\left(\Theta_{\mathfrak{a}, \chi}^{m}\right)=\int_{K^{1} \backslash K_{\mathbf{A}}^{1}} \Theta_{\mathfrak{a}, \chi}^{m}(t) d^{\times} t
$$

is the period of $\Theta_{\mathfrak{a}, \chi}^{m}$ and $J(m, \mathfrak{a}, \chi, f)$ is a linear combination of $W_{\chi, f}^{m}\left(h_{j, m, \mathfrak{a}}\right)$ with some $h_{j, m, \mathfrak{a}} \in H_{\mathbf{A}}$. (Note that $J(m, \mathfrak{a}, \chi, f)$ is a linear combination of 'CM-values' of $f$; see the remark in $\S 7.6$.)

Main Theorem II (Theorem 7.6). Suppose that $f$ is a Hecke eigenform. Then, for each $(m, \mathfrak{a}, \chi)$, $\left\{C_{\mathcal{L} f}\left(\mathfrak{b} ; \Theta_{\mathfrak{a}, \chi}^{m}\right) \mid \mathfrak{b} \subset \mathfrak{a}\right\}$ satisfies certain recursion relations. This implies that $\mathcal{L} f$ is a Hecke eigenform and $L(\mathcal{L} f, \xi ; s)=L(\xi ; s) L(f, \xi ; s)$ for a Hecke character $\xi$ of $K$ (Corollary 7.7).

Main Theorem III (Theorem 7.8). Suppose that $f$ is a Hecke eigenform. Then $\mathcal{L} f \neq 0$ if and only if there exists a triplet $(m, \mathfrak{a}, \chi)$ such that $L(\chi ; 1 / 2) J(m, \mathfrak{a}, \chi, f) \neq 0$.

Remark. It should be noted that Gelbart, Rogawski and Soudry [GRS97] studied the nonvanishing of a theta lift from $U(2)$ to $U(3)$ in a representation theoretic setting (see also [Tan99]). To be more precise, let $\sigma=\bigotimes_{v} \sigma_{v}$ be a cuspidal representation of $U(2)$, and suppose that the theta lift of $\sigma$ to $U(1)$ is zero. Then they showed that the theta lift of $\sigma$ given by theta integrals is nonzero precisely when the local Howe lift of each component $\sigma_{v}$ is nonzero. On the other hand, our criterion is concerned with the nonvanishing of the theta lift of an automorphic form (an element of the space of the automorphic representation), and is derived from the study of the Fourier-Jacobi expansion of the theta lift.

Remark. The inner product formula for the unitary Kudla lift has been given in [MS06].

1.5 The paper is organized as follows. The first five sections are of a preliminary nature. After fixing the notation in $\S 2$, we define automorphic forms on $U(1,1)$ and $U(2,1)$ in $\S 3$. In $\S 4$, we recall several fundamental properties of theta functions on $R$ after Shintani's work [Shi79] and our preceding papers [MS00, MS02]. In $\S 5$, we review Shintani's theory of the Fourier-Jacobi expansion of holomorphic automorphic forms on $U(2,1)$ and reformulate Shintani's result mentioned above. In $\S 6$, we study metaplectic representations of $U(2,1) \times U(1,1)$. We use a mixed model of the metaplectic representation, which is more convenient in the calculation of the Fourier-Jacobi components than the Schrödinger model. The intertwining operator between two models is given in $\S 6.6$. In $\S 7$, we define the unitary Kudla lift and state the main results of the paper (Theorems 7.5, 7.6 and 7.8), whose proofs occupy the remaining sections. The key of the proofs is Lemma 8.3, in which we use the properties of metaplectic representations in an essential way. By using this, we obtain a formula relating the Fourier-Jacobi components of $\mathcal{L} f$ to certain integrals of spherical functions $W_{\chi, f}^{m}$ in $\S 8$ (Proposition 8.4). Using this and the results of $\S 5$, we give another proof of (1.1) in $\S 9$. In $\S 10$, we reduce the proof of Theorems 7.5 and 7.6 to the calculation of certain local integrals introduced in this section (see Theorems 10.1 and 10.2). The calculations are carried out in $\S 11$ for the primitive case and in $\S 12$ for the nonprimitive case. 


\section{A. Murase And T. Sugano}

\section{Notation}

2.1 An index of notation is given in Table 1 . As usual, $\mathbb{Z}, \mathbb{Q}, \mathbb{R}$ and $\mathbb{C}$ denote the ring of rational integers, the rational number field, the field of real and complex numbers, respectively. For $z \in \mathbb{C}$ and $m \in \mathbb{Q}$, put $\mathbf{e}[z]=\exp (2 \pi \sqrt{-1} z)$ and $\mathbf{e}_{m}[z]=\mathbf{e}[m z]$. For a finite prime $p$, let $\mathbb{Z}_{p}$ be the $p$-adic integer ring and $\mathbb{Q}_{p}$ the $p$-adic number field. We put $\mathbb{Z}_{f}=\prod_{p<\infty} \mathbb{Z}_{p}$. Let $\mathbb{Q}_{\mathbf{A}}$ denote the adele ring of $\mathbb{Q}$. For a prime $v$ of $\mathbb{Q},|\cdot|_{v}$ stands for the absolute value of $\mathbb{Q}_{v}^{\times}$. When $v=p<\infty$, the normalized additive valuation $\operatorname{ord}_{p}: \mathbb{Q}_{p}^{\times} \rightarrow \mathbb{Z}$ is given by $|a|_{p}=p^{-\operatorname{ord}_{p} a}\left(a \in \mathbb{Q}_{p}^{\times}\right)$. We denote by $|a|_{\mathbf{A}}=\prod_{v}\left|a_{v}\right|_{v}$ the idéle norm of $a=\left(a_{v}\right)_{v} \in \mathbb{Q}_{\mathbf{A}}^{\times}$. Let $\psi$ be the additive character of $\mathbb{Q}_{\mathbf{A}} / \mathbb{Q}$ satisfying $\psi\left(x_{\infty}\right)=\mathbf{e}\left[x_{\infty}\right]\left(x_{\infty} \in \mathbb{R}\right)$. For $m \in \mathbb{Q}$, we put $\psi_{m}(x)=\psi(m x)$ for $x \in \mathbb{Q}_{\mathbf{A}}$. Let $\psi_{v}$ (respectively $\psi_{m, v}$ ) be the restriction of $\psi\left(\right.$ respectively $\psi_{m}$ ) to $\mathbb{Q}_{v}$ for each prime $v$ of $\mathbb{Q}$.

Let $X$ be a linear algebraic group defined over $\mathbb{Q}$. For a prime $v$ of $\mathbb{Q}, X_{v}$ stands for the group of $\mathbb{Q}_{v}$-rational points of $X$. Denote by $X_{\mathbf{A}}$ and $X_{\mathbf{A}, f}$ the adélization of $X$ and its finite part, respectively.

Let $\operatorname{diag}\left(a_{1}, \ldots, a_{n}\right)$ be the diagonal matrix of degree $n$ with the $i$ th diagonal component $a_{i}$ for $i=1, \ldots, n$. For $x \in \mathbb{R}$, we write $[x]$ for the integer with $0 \leqslant x-[x]<1$. If $P$ is a condition, we put $\delta(P)=1$ if $P$ holds, and $\delta(P)=0$ otherwise. For a set $C$, char $C$ denotes the characteristic function of $C$.

2.2 Let $K$ be an imaginary quadratic field of discriminant $D$ with integer ring $\mathcal{O}_{K}$. We fix an embedding of $K$ into $\mathbb{C}$ and let $\kappa=\sqrt{D}$ so that $\operatorname{Im}(\kappa)>0$. Denote by $\sigma$ the nontrivial automorphism of $K / \mathbb{Q}$. For $z \in K$, we write $\operatorname{Tr}(z)$ and $\mathrm{N}(z)$ for $\operatorname{Tr}_{K / \mathbb{Q}}(z)=z+z^{\sigma}$ and $\mathrm{N}_{K / \mathbb{Q}}(z)=z z^{\sigma}$, respectively, if there is no fear of confusion. We put $\psi_{K}(z)=\psi(\operatorname{Tr}(z))\left(z \in K_{\mathbf{A}}\right)$. For a prime $v$ of $\mathbb{Q}$, let $K_{v}=K \otimes_{\mathbb{Q}} \mathbb{Q}_{v}$. For a finite prime $p$, put $\mathcal{O}_{K, p}=\mathcal{O}_{K} \otimes_{\mathbb{Z}} \mathbb{Z}_{p}$. We set $\mathcal{O}_{K, f}=\prod_{p<\infty} \mathcal{O}_{K, p}$. By an ideal of $K$, we always mean a nonzero fractional ideal of $K$. For an ideal $\mathfrak{a}$ of $K$ and a finite prime $p$, we put $\mathfrak{a}_{p}=\mathfrak{a} \otimes_{\mathcal{O}_{K}} \mathcal{O}_{K, p}$. Let $\mathfrak{a}_{f}=\mathfrak{a} \otimes_{\mathcal{O}_{K}} \mathcal{O}_{K, f}=\prod_{p<\infty} \mathfrak{a}_{p}$. For $\alpha=\alpha_{\infty} \alpha_{f} \in K_{\mathbf{A}}^{\times}$, we denote by $\operatorname{id}(\alpha)$ the ideal $\mathfrak{a}$ of $K$ determined by $\mathfrak{a}_{f}=\alpha_{f} \mathcal{O}_{K, f}$. Let $K^{1}=\left\{t \in K^{\times} \mid t t^{\sigma}=1\right\}$. We put $\mathcal{O}_{K, f}^{1}=K_{\mathbf{A}, f}^{1} \cap \mathcal{O}_{K, f}^{\times}=\prod_{p<\infty} \mathcal{O}_{K, p}^{1}$, where $\mathcal{O}_{K, p}^{1}=K_{p}^{1} \cap \mathcal{O}_{K, p}^{\times}$. When $p$ splits in $K / \mathbb{Q}$, we fix an identification $K_{p}$ with $\mathbb{Q}_{p} \oplus \mathbb{Q}_{p}$, and put $\Pi_{p, 1}=(p, 1)$ and $\Pi_{p, 2}=(1, p)$. When $p$ ramifies in $K / \mathbb{Q}$ (namely $p \mid D$ ), we fix a prime element $\Pi_{p}$ of $K_{p}$ and put $\pi_{p}=\mathrm{N}\left(\Pi_{p}\right)$. When $p$ is inert or splits in $K / \mathbb{Q}$, we take and fix a prime element $\pi_{p}$ of $\mathbb{Q}_{p}$.

Let $\theta$ be an element of $\mathcal{O}_{K}$ such that $\mathcal{O}_{K}=\mathbb{Z}+\mathbb{Z} \theta, \theta-\theta^{\sigma}=\kappa$ and $\operatorname{ord}_{p} \mathrm{~N}_{K / \mathbb{Q}}(\theta)=1$ for every $p$ ramified in $K / \mathbb{Q}$. For example, we can take $\theta$ as

$$
\theta=\frac{D^{\prime}+\sqrt{D}}{2}, \quad D^{\prime}= \begin{cases}D & \text { if } D \text { is odd } \\ D / 2 & \text { if } D \text { is even }\end{cases}
$$

For $A \in M_{m, n}(K)$, put $A^{*}={ }^{\mathrm{t}} A^{\sigma}$. Let $A \in M_{n}(K)$ be a hermitian (respectively an anti-hermitian) matrix of degree $n$, namely $A^{*}=A$ (respectively $A^{*}=-A$ ). Let $W=K^{n}$ be the $K$-vector space of column vectors of dimension $n$ coefficients in $K$. For $w, w^{\prime} \in W$, we put $A\left(w, w^{\prime}\right)=w^{*} A w^{\prime}$ and $A[w]=A(w, w)$. If $A$ is anti-hermitian, we define an alternating $\mathbb{Q}$-form $\langle\cdot, \cdot\rangle_{A}$ on $W$ by $\left\langle w, w^{\prime}\right\rangle_{A}=\operatorname{Tr}\left(w^{*} A w^{\prime}\right)\left(w, w^{\prime} \in W\right)$.

For a prime $v$ of $\mathbb{Q}$, we denote by $\mathcal{S}\left(K_{v}^{m}\right)$ the space of Schwartz-Bruhat functions on $K_{v}^{m}$.

2.3 Let

$$
S=\left[\begin{array}{ccc} 
& 1 & \\
-\kappa^{-1} & &
\end{array}\right], \quad T=\left(\begin{array}{rr}
0 & 1 \\
-1 & 0
\end{array}\right)
$$


TABLE 1. Index of notation.

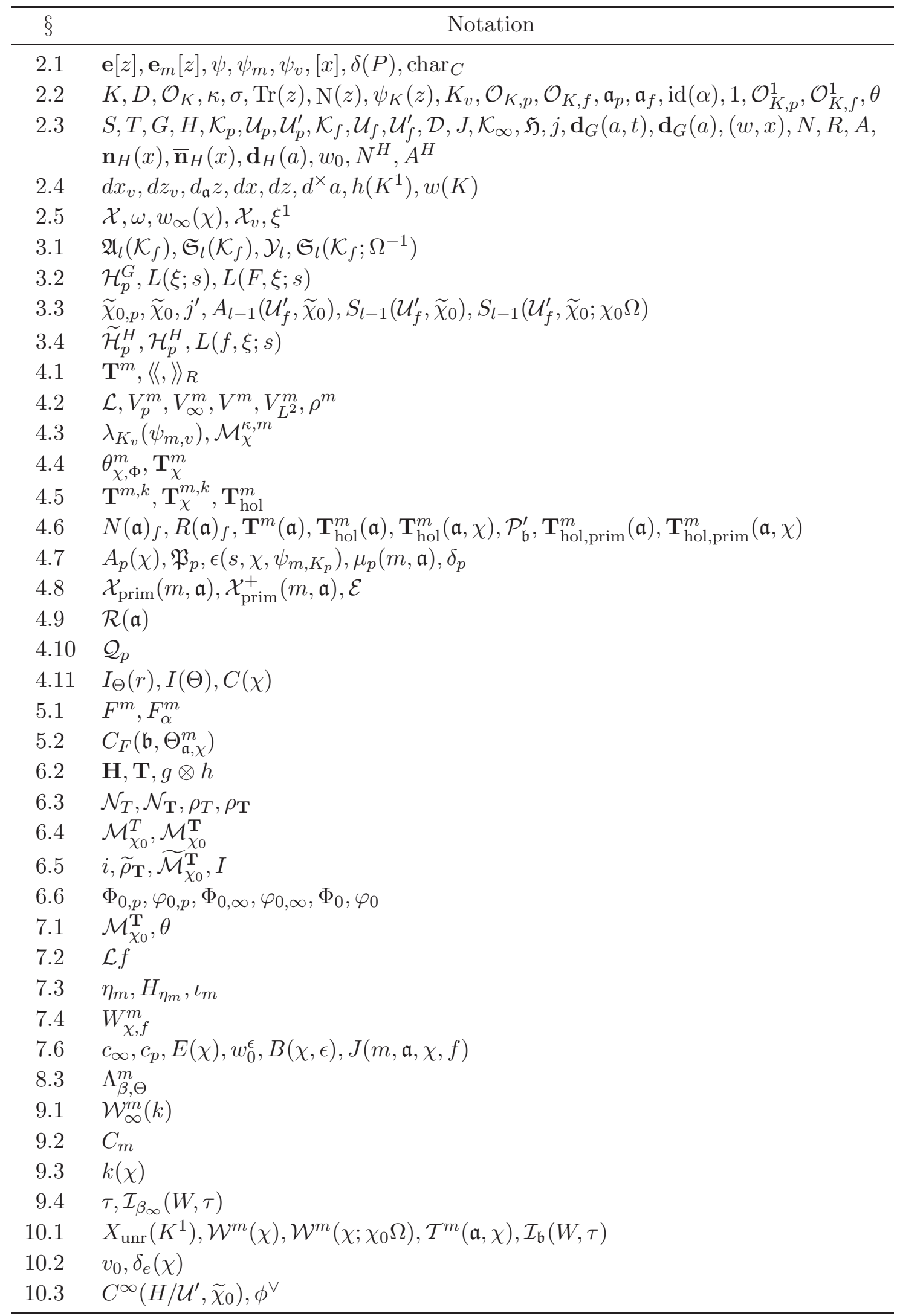




\section{A. Murase And T. Sugano}

Then $S$ (respectively $\sqrt{-1}^{-1} T$ ) is a hermitian matrix of signature $(2,1)$ (respectively $(1,1)$ ). Denote by $G$ and $H$ the unitary groups of $S$ and $T$, respectively. By definition, $G_{\mathbb{Q}}=\left\{g \in \mathrm{GL}_{3}(K) \mid\right.$ $\left.g^{*} S g=S\right\}$ and $H_{\mathbb{Q}}=\left\{h \in \mathrm{GL}_{2}(K) \mid h^{*} T h=T\right\}$. We put

$$
\mathcal{K}_{p}=G_{p} \cap \mathrm{GL}_{3}\left(\mathcal{O}_{K, p}\right), \quad \mathcal{U}_{p}=H_{p} \cap \mathrm{GL}_{2}\left(\mathcal{O}_{K, p}\right), \quad \mathcal{U}_{p}^{\prime}=\left\{\left(\begin{array}{ll}
a & b \\
c & d
\end{array}\right) \in \mathcal{U}_{p} \mid c \in D \mathcal{O}_{K, p}\right\} .
$$

Then $\mathcal{K}_{p}$ (respectively $\mathcal{U}_{p}$ ) is a maximal open compact subgroup of $G_{p}$ (respectively $H_{p}$ ), and $\mathcal{U}_{p}^{\prime}$ is an open subgroup of $\mathcal{U}_{p}$. Note that $\mathcal{U}_{p}=\mathcal{U}_{p}^{\prime}$ unless $p \mid D$. We set

$$
\mathcal{K}_{f}=\prod_{p<\infty} \mathcal{K}_{p}, \quad \mathcal{U}_{f}=\prod_{p<\infty} \mathcal{U}_{p}, \quad \mathcal{U}_{f}^{\prime}=\prod_{p<\infty} \mathcal{U}_{p}^{\prime}
$$

Let

$$
\mathcal{D}=\left\{\left(\begin{array}{c}
z \\
w
\end{array}\right) \in \mathbb{C}^{2} \mid \frac{z-\bar{z}}{\kappa}-w \bar{w}>0\right\}
$$

be a hermitian symmetric domain isomorphic to the complex two ball. The real points $G_{\infty}$ of $G$ acts on $\mathcal{D}$ as follows. For $g \in G_{\infty}$ and $Z={ }^{\mathrm{t}}(z, w) \in \mathcal{D}$, there exist $g\langle Z\rangle \in \mathcal{D}$ and $J(g, Z) \in \mathbb{C}^{\times}$ such that $g \cdot Z^{\sim}=J(g, Z) \cdot(g\langle Z\rangle)^{\sim}$, where $Z^{\sim}={ }^{\mathrm{t}}(z, w, 1) \in \mathbb{C}^{3}$. Then $(g, Z) \mapsto g\langle Z\rangle$ defines a holomorphic action of $G_{\infty}$ on $\mathcal{D}$ and $J: G_{\infty} \times \mathcal{D} \rightarrow \mathbb{C}^{\times}$is a holomorphic automorphic factor. Let $\mathcal{K}_{\infty}=\left\{g \in G_{\infty} \mid g_{\infty}\left\langle Z_{0}\right\rangle=Z_{0}\right\}$ be the stabilizer of $Z_{0}={ }^{\mathrm{t}}(\kappa / 2,0) \in \mathcal{D}$ in $G_{\infty}$. It is known that $\mathcal{K}_{\infty}=G_{\infty} \cap U\left(S_{0}\right)$ with $S_{0}=\operatorname{diag}(-2 / D, 1,1 / 2)$.

We define the action of $H_{\infty}$ on the upper half plane $\mathfrak{H}=\{z \in \mathbb{C} \mid \operatorname{Im}(z)>0\}$ and the holomorphic automorphic factor $j: H_{\infty} \times \mathfrak{H} \rightarrow \mathbb{C}^{\times}$in a usual way:

$$
h\langle z\rangle=\frac{a z+b}{c z+d}, \quad j(h, z)=c z+d
$$

for $h=\left(\begin{array}{ll}a & b \\ c & d\end{array}\right) \in H_{\infty}$ and $z \in \mathfrak{H}$. Denote by $\mathcal{U}_{\infty}$ the stabilizer of $z_{0}=\sqrt{-1} \in \mathfrak{H}$ in $H_{\infty}$.

We put $\mathbf{d}_{G}(a, t)=\operatorname{diag}\left(a^{\sigma}, t, a^{-1}\right)$ and $\mathbf{d}_{G}(a)=\mathbf{d}_{G}(a, 1)$ for $a \in K^{\times}, t \in K^{1}$, and

$$
(w, x)=\left[\begin{array}{ccc}
1 & \kappa w^{\sigma} & x+\frac{\kappa}{2} w w^{\sigma} \\
& w \\
& & 1
\end{array}\right] \quad(w \in K, x \in \mathbb{Q}) .
$$

Note that $(w, x)\left(w^{\prime}, x^{\prime}\right)=\left(w+w^{\prime}, x+x^{\prime}+\frac{1}{2}\left\langle w, w^{\prime}\right\rangle_{\kappa}\right)$ and $\mathbf{d}_{G}(a, t) \cdot(w, x) \cdot \mathbf{d}_{G}(a, t)^{-1}=(a t w, \mathrm{~N}(a) x)$, where $\left\langle w, w^{\prime}\right\rangle_{\kappa}=\operatorname{Tr}\left(\kappa w^{\sigma} w^{\prime}\right)$. We write $n t$ for $n \cdot \mathbf{d}_{G}(1, t)$ if there is no fear of confusion (the scalar matrix $\operatorname{diag}(t, t, t)$ is always denoted by $t 1_{3}$ throughout the paper). Define subgroups $N, R$ and $A$ of $G$ by $N_{\mathbb{Q}}=\{(w, x) \mid w \in K, x \in \mathbb{Q}\}, R_{\mathbb{Q}}=\left\{n t \mid n \in N_{\mathbb{Q}}, t \in K^{1}\right\}$ and $A_{\mathbb{Q}}=\left\{\mathbf{d}_{G}(a) \mid a \in K^{\times}\right\}$. We see that $N_{p} \cap \mathcal{K}_{p}=\left\{\left(w, x+x_{w}\right) \mid w \in \mathcal{O}_{K, p}, x \in \mathbb{Z}_{p}\right\}$, where $x_{w}=2^{-1} \operatorname{Tr} \theta w w^{\sigma}(w \in K)$.

Put

$$
\mathbf{n}_{H}(x)=\left(\begin{array}{ll}
1 & x \\
0 & 1
\end{array}\right), \quad \overline{\mathbf{n}}_{H}(x)=\left(\begin{array}{ll}
1 & 0 \\
x & 1
\end{array}\right) \quad(x \in \mathbb{Q})
$$

and

$$
\mathbf{d}_{H}(a)=\left(\begin{array}{cc}
a^{\sigma} & 0 \\
0 & a^{-1}
\end{array}\right) \quad\left(a \in K^{\times}\right), \quad w_{0}=\left(\begin{array}{rr}
0 & 1 \\
-1 & 0
\end{array}\right) .
$$

Define subgroups $N^{H}$ and $A^{H}$ of $H$ by $N_{\mathbb{Q}}^{H}=\left\{\mathbf{n}_{H}(x) \mid x \in \mathbb{Q}\right\}$ and $A_{\mathbb{Q}}^{H}=\left\{\mathbf{d}_{H}(a) \mid a \in K^{\times}\right\}$.

We have the Iwasawa decompositions:

$$
G_{\mathbf{A}}=R_{\mathbf{A}} A_{\mathbf{A}} \mathcal{K}_{f} \mathcal{K}_{\infty}, \quad H_{\mathbf{A}}=N_{\mathbf{A}}^{H} A_{\mathbf{A}}^{H} \mathcal{U}_{f} \mathcal{U}_{\infty}
$$




\section{ON THE Fourier-JACOBI EXPANSION OF THE UNITARY KUDLA LIFT}

2.4 In this subsection, we normalize Haar measures on various groups. Let $v$ be a prime of $\mathbb{Q}$. Let $d x_{v}$ be the Haar measure on $\mathbb{Q}_{v}$ self-dual with respect to the pairing $\left(x_{v}, x_{v}^{\prime}\right) \mapsto \psi_{v}\left(x_{v} x_{v}^{\prime}\right)$. Note that $\int_{\mathbb{Z}_{p}} d x_{p}=1$ for $p<\infty$ and that $d x_{\infty}$ is the usual Lebesgue measure on $\mathbb{R}$. Let $d z_{v}$ be the Haar measure on $K_{v}$ self-dual with respect to the pairing $\left(z_{v}, z_{v}^{\prime}\right) \mapsto \psi_{K}\left(z_{v}^{\sigma} z_{v}^{\prime}\right)$. Note that $\int_{\mathcal{O}_{K, p}} d z_{p}=|D|_{p}^{1 / 2}$ and $d z_{\infty}=2 d_{L} z_{\infty}$, where $d_{L} z_{\infty}$ is the usual Lebesgue measure on $\mathbb{C}$. For an ideal $\mathfrak{a}$ of $K_{p}, d_{\mathfrak{a}} z$ stands for the Haar measure on $K_{p}$ normalized by $\operatorname{vol}(\mathfrak{a})=1$.

Let $d x=\prod_{v} d x_{v}$ and $d z=\prod_{v} d z_{v}$. Then $d x$ and $d z$ are the Haar measures on $\mathbb{Q}_{\mathbf{A}}$ and $K_{\mathbf{A}}$ with $\operatorname{vol}\left(\mathbb{Q} \backslash \mathbb{Q}_{\mathbf{A}}\right)=1$ and $\operatorname{vol}\left(K \backslash K_{\mathbf{A}}\right)=1$, respectively. Let $d^{\times} a=\prod_{v} d^{\times} a_{v}$ be the Haar measure on $K_{\mathbf{A}}^{\times}$, where $d^{\times} a_{v}$ is the Haar measure on $K_{v}^{\times}$normalized by $\operatorname{vol}\left(\mathcal{O}_{K, p}^{\times}\right)=1$ if $v=p<\infty$, and $d^{\times} a_{\infty}=d a_{\infty} / \mathrm{N}\left(a_{\infty}\right)$. Let $d^{\times} t=\prod_{v} d^{\times} t_{v}$ be the Haar measure on $K_{\mathbf{A}}^{1}$, where $d^{\times} t_{v}$ is the Haar measure on $K_{v}^{1}$ normalized by $\operatorname{vol}\left(\mathcal{O}_{K, p}^{1}\right)=1$ if $v=p<\infty$, and $\operatorname{vol}\left(K_{\infty}^{1}\right)=1$ if $v=\infty$. Under this normalization, we have $\operatorname{vol}\left(K^{1} \backslash K_{\mathbf{A}}^{1}\right)=h\left(K^{1}\right) / w(K)$, where $h\left(K^{1}\right)=\#\left(K^{1} \backslash K_{\mathbf{A}}^{1} / K_{\infty}^{1} \mathcal{O}_{K, f}^{1}\right)$ is the class number of $K^{1}$ and $w(K)$ is the number of roots of unity in $K$.

The Haar measures on $N_{v}$ and $R_{v}$ are given as follows:

$$
\begin{gathered}
d n_{v}=d w_{v} d x_{v} \quad\left(n_{v}=\left(w_{v}, x_{v}\right), w_{v} \in K_{v}, x_{v} \in \mathbb{Q}_{v}\right), \\
d r_{v}=d n_{v} d^{\times} t_{v} \quad\left(r_{v}=n_{v} t_{v}, n_{v} \in N_{v}, t_{v} \in K_{v}^{1}\right) .
\end{gathered}
$$

When $v=p<\infty$, we normalize Haar measures $d g_{p}$ and $d h_{p}$ on $G_{p}$ and $H_{p}$ by

$$
\int_{\mathcal{K}_{p}} d g_{p}=1, \quad \int_{\mathcal{U}_{p}} d h_{p}=1
$$

When $v=\infty$, we normalize Haar measures $d g_{\infty}$ and $d h_{\infty}$ on $G_{\infty}$ and $H_{\infty}$ by

$$
\int_{G_{\infty}} \Phi\left(g_{\infty}\right) d g_{\infty}=\int_{R_{\infty}} \int_{\mathbb{C}^{\times}} \int_{\mathcal{K}_{\infty}}\left|N\left(a_{\infty}\right)\right|_{\infty}^{-2} \Phi\left(r_{\infty} \mathbf{d}_{G}\left(a_{\infty}\right) k_{\infty}\right) d k_{\infty} d^{\times} a_{\infty} d r_{\infty} \quad\left(\Phi \in L^{1}\left(G_{\infty}\right)\right)
$$

and

$$
\int_{H_{\infty}} \varphi\left(h_{\infty}\right) d h_{\infty}=\int_{\mathbb{R}} \int_{\mathbb{C}^{\times}} \int_{\mathcal{U}_{\infty}}\left|N\left(a_{\infty}\right)\right|_{\infty}^{-1} \varphi\left(\mathbf{n}_{H}(x) \mathbf{d}_{H}\left(a_{\infty}\right) u_{\infty}\right) d u_{\infty} d^{\times} a_{\infty} d x_{\infty} \quad\left(\varphi \in L^{1}\left(H_{\infty}\right)\right) .
$$

Here $d k_{\infty}$ (respectively $d u_{\infty}$ ) is normalized by $\operatorname{vol}\left(\mathcal{K}_{\infty}\right)=1$ (respectively $\operatorname{vol}\left(\mathcal{U}_{\infty}\right)=1$ ). Finally, let $d n=\prod_{v} d n_{v}, d r=\prod_{v} d r_{v}, d g=\prod_{v} d g_{v}$ and $d h=\prod_{v} d h_{v}$ be the Haar measures on $N_{\mathbf{A}}, R_{\mathbf{A}}, G_{\mathbf{A}}$ and $H_{\mathbf{A}}$, respectively.

2.5 Let $\mathcal{X}$ be the set of unitary Hecke characters $\chi$ of $K$ satisfying $\left.\chi\right|_{\mathbb{Q}_{\mathbf{A}}^{\times}}=\omega$, where $\omega=\omega_{K / \mathbb{Q}}$ is the quadratic Hecke character of $\mathbb{Q}$ corresponding to $K / \mathbb{Q}$. For $\chi \in \mathcal{X}$, let $w_{\infty}(\chi)$ be the integer such that $\chi\left(z_{\infty}\right)=\left(z_{\infty} /\left|z_{\infty}\right|\right)^{w_{\infty}(\chi)}$ for $z_{\infty} \in K_{\infty}^{\times}$. Note that $w_{\infty}(\chi)$ is always odd. For a prime $v$ of $\mathbb{Q}$, let $\mathcal{X}_{v}$ be the set of the set of unitary characters $\chi$ of $K_{v}^{\times}$with $\left.\chi\right|_{\mathbb{Q}_{v}^{\times}}=\omega_{v}$. For a Hecke character $\xi$ of $K$ trivial on $\mathbb{Q}_{\mathbf{A}}^{\times}$, we define a character $\xi^{1}$ of $K_{\mathbf{A}}^{1} / K^{1}$ by $\xi^{1}\left(z^{\sigma} / z\right)=\xi(z)\left(z \in K_{\mathbf{A}}^{\times}\right)$.

\section{Automorphic forms on $U(2,1)$ and $U(1,1)$}

\subsection{Automorphic forms on $U(2,1)$}

We henceforth fix a positive integer $l$ divisible by $w(K)$. We first recall the definition of holomorphic automorphic forms on $G=U(S)$. Let $\mathfrak{A}_{l}\left(\mathcal{K}_{f}\right)$ be the space of smooth functions $F$ on $G_{\mathbb{Q}} \backslash G_{\mathbf{A}}$ satisfying:

(i) $F\left(g k_{f} k_{\infty}\right)=J\left(k_{\infty}, Z_{0}\right)^{-l} F(g)\left(g \in G_{\mathbf{A}}, k_{f} \in \mathcal{K}_{f}, k_{\infty} \in \mathcal{K}_{\infty}\right)$;

(ii) for any $g_{f} \in G_{\mathbf{A}, f}, g_{\infty}\left\langle Z_{0}\right\rangle \mapsto J\left(g_{\infty}, Z_{0}\right)^{l} F\left(g_{f} g_{\infty}\right)$ is holomorphic on $\mathcal{D}$. 


\section{A. Murase And T. Sugano}

Denote by $\mathfrak{S}_{l}\left(\mathcal{K}_{f}\right)$ the space of $F \in \mathfrak{A}_{l}\left(\mathcal{K}_{f}\right)$ satisfying

$$
\int_{{\mathbb{Q} \backslash \mathbb{Q}_{\mathbf{A}}}} F((0, x) g) d x=0 \quad\left(g \in G_{\mathbf{A}}\right) .
$$

We call $\mathfrak{A}_{l}\left(\mathcal{K}_{f}\right)$ (respectively $\mathfrak{S}_{l}\left(\mathcal{K}_{f}\right)$ ) the space of holomorphic automorphic forms (respectively holomorphic cusp forms) of weight $l$ on $\mathcal{K}_{f}$. It is known that $F \in \mathfrak{S}_{l}\left(\mathcal{K}_{f}\right)$ is bounded on $G_{\mathbf{A}}$.

Let $\mathcal{Y}_{l}$ be the set of unitary characters $\Omega$ of $K_{\mathbf{A}}^{1} / K^{1}$ satisfying $\left.\Omega\right|_{\mathcal{O}_{K, f}^{1}}=1$ and $\Omega\left(z_{\infty}\right)=z_{\infty}^{l}$ for $z_{\infty} \in K_{\infty}^{1}$. For $\Omega \in \mathcal{Y}_{l}$, let $\mathfrak{S}_{l}\left(\mathcal{K}_{f} ; \Omega^{-1}\right)$ be the space of $F \in \mathfrak{S}_{l}\left(\mathcal{K}_{f}\right)$ satisfying $F\left(t 1_{3} \cdot g\right)=\Omega^{-1}(t) F(g)$ for $t \in K_{\mathbf{A}}^{1}$. We then have $\mathfrak{S}_{l}\left(\mathcal{K}_{f}\right)=\bigoplus_{\Omega \in \mathcal{Y}_{l}} \mathfrak{S}_{l}\left(\mathcal{K}_{f} ; \Omega^{-1}\right)$.

\subsection{Hecke operators and $L$-functions for $U(2,1)$}

For $p<\infty$, let $\mathcal{H}_{p}^{G}=\mathcal{H}\left(G_{p}, \mathcal{K}_{p}\right)$ be the Hecke algebra of $\left(G_{p}, \mathcal{K}_{p}\right)$. By definition, $\mathcal{H}_{p}^{G}$ is the space of compactly supported bi- $\mathcal{K}_{p}$-invariant functions on $G_{p}$ and the product is given by

$$
\left(\Phi * \Phi^{\prime}\right)(g)=\int_{G_{p}} \Phi\left(g x^{-1}\right) \Phi^{\prime}(x) d x \quad\left(\Phi, \Phi^{\prime} \in \mathcal{H}_{p}^{G}\right) .
$$

The $\mathbb{C}$-algebra $\mathcal{H}_{p}^{G}$ acts on $\mathfrak{S}_{l}\left(\mathcal{K}_{f} ; \Omega^{-1}\right)$ by

$$
(F * \Phi)(g)=\int_{G_{p}} F\left(g x^{-1}\right) \Phi(x) d x \quad\left(F \in \mathfrak{S}_{l}\left(\mathcal{K}_{f} ; \Omega^{-1}\right), \Phi \in \mathcal{H}_{p}^{G}\right) .
$$

The following facts are elementary (cf. [Shi79, Lemma 2] and [Kud81, p. 333]).

Lemma 3.1. Let $F \in \mathfrak{S}_{l}\left(\mathcal{K}_{f} ; \Omega^{-1}\right)$.

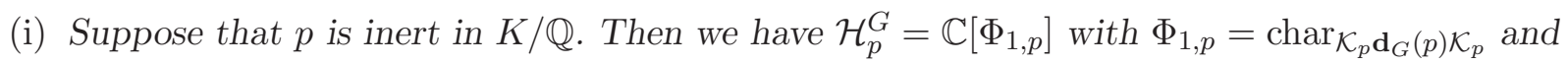

$$
\begin{aligned}
\left(F * \Phi_{1, p}\right)(g)= & F\left(g \mathbf{d}_{G}\left(p^{-1}\right)\right)+\sum_{x \in\left(\mathbb{Z}_{p}-p \mathbb{Z}_{p}\right) / p \mathbb{Z}_{p}} F\left(g\left(0, p^{-1} x\right)\right) \\
& +\sum_{w \in \mathcal{O}_{K, p} / p \mathcal{O}_{K, p}} \sum_{x \in \mathbb{Z}_{p} / p^{2} \mathbb{Z}_{p}} F\left(g\left(w, x+x_{w}\right) \mathbf{d}_{G}(p)\right) .
\end{aligned}
$$

(ii) Suppose that $p$ ramifies in $K / \mathbb{Q}$ and let $\Pi$ be a prime element of $K_{p}$. Then we have $\mathcal{H}_{p}^{G}=\mathbb{C}\left[\Phi_{1, p}\right]$ with $\Phi_{1, p}=\operatorname{char}_{\mathcal{K}_{p} \mathbf{d}_{G}(\Pi) \mathcal{K}_{p}}$ and

$$
\begin{aligned}
\left(F * \Phi_{1, p}\right)(g)= & F\left(g \mathbf{d}_{G}\left(\Pi^{-1}\right)\right)+\sum_{w \in\left(\mathcal{O}_{K, p}-\Pi \mathcal{O}_{K, p}\right) / \Pi \mathcal{O}_{K, p}} F\left(g\left(\Pi^{-1} w, x_{\Pi^{-1} w}\right)\right) \\
& +\sum_{w \in \mathcal{O}_{K, p} / \Pi \mathcal{O}_{K, p}} \sum_{x \in \mathbb{Z}_{p} / p \mathbb{Z}_{p}} F\left(g\left(w, x+x_{w}\right) \mathbf{d}_{G}(\Pi)\right) .
\end{aligned}
$$

(iii) Suppose that $p$ splits in $K / \mathbb{Q}$ and put $\Pi_{1}=(p, 1)$ and $\Pi_{2}=(1, p)$. Then we have $\mathcal{H}_{p}^{G}=$ $\mathbb{C}\left[\Phi_{1, p}, \Phi_{2, p}, \Phi_{0, p}^{ \pm}\right]$, where $\Phi_{i, p}=\operatorname{char}_{\mathcal{K}_{p} \mathbf{d}_{G}\left(\Pi_{i}\right) \mathcal{K}_{p}}(i=1,2), \Phi_{0, p}^{ \pm}=\operatorname{char}_{\mathcal{K}_{p}\left(\Pi_{1} / \Pi_{2}\right)^{ \pm 1} 1_{3} \mathcal{K}_{p}}$, and

$$
\begin{aligned}
\left(F * \Phi_{i, p}\right)(g)= & F\left(g \mathbf{d}_{G}\left(\Pi_{i}^{-1}\right)\right)+\sum_{w \in \mathcal{O}_{K, p} / \Pi_{i} \mathcal{O}_{K, p}} F\left(g\left(w, x_{w}\right)\left(\Pi_{i} / \Pi_{i}^{\sigma}\right)\right) \\
& +\sum_{w \in \mathcal{O}_{K, p} / \Pi_{i}^{\sigma} \mathcal{O}_{K, p}} \sum_{x \in \mathbb{Z}_{p} / p \mathbb{Z}_{p}} F\left(g\left(w, x+x_{w}\right) \mathbf{d}_{G}\left(\Pi_{i}^{\sigma}\right)\right) \quad(i=1,2), \\
\left(F * \Phi_{0, p}^{ \pm}\right)(g)= & F\left(g \cdot\left(\Pi_{1} / \Pi_{2}\right)^{\mp 1} 1_{3}\right) \quad\left(=\Omega^{ \pm 1}\left(\Pi_{1} / \Pi_{2}\right) F(g)\right) .
\end{aligned}
$$

If, for every $p<\infty$, there exists a $\mathbb{C}$-algebra homomorphism $\Lambda_{p}$ of $\mathcal{H}_{p}^{G}$ to $\mathbb{C}$ such that $F * \Phi=$ $\Lambda_{p}(\Phi) F$ holds for any $\Phi \in \mathcal{H}_{p}^{G}$, we say that $F$ is a Hecke eigenform with eigenvalues $\left\{\Lambda_{p}\right\}$. 


\section{ON THE Fourier-JACOBI EXPANSION OF THE UNITARY KUDLA LIFT}

Let $F \in \mathfrak{S}_{l}\left(\mathcal{K}_{f} ; \Omega^{-1}\right)$ be a Hecke eigenform with eigenvalues $\left\{\Lambda_{p}\right\}$ and $\xi$ a Hecke character of $K$. Let $L(\xi ; s)=\prod_{p<\infty} L_{p}(\xi ; s)$ be the Hecke $L$-function of $\xi$. We define an $L$-function

$$
L(F, \xi ; s)=\prod_{p<\infty} L_{p}(F, \xi ; s)
$$

where the local factor $L_{p}(F, \xi ; s)$ is given as follows.

(i) If $p$ is inert in $K / \mathbb{Q}$, put

$$
L_{p}(F, \xi ; s)=L_{p}(\xi ; s) Q_{p}\left(\xi_{p}(p) p^{-2 s}\right)^{-1}
$$

where

$$
Q_{p}(X)=1-p^{-2}\left\{\Lambda_{p}\left(\Phi_{1, p}\right)-p+1\right\} X+X^{2} .
$$

(Here we make a convention that $\xi_{p}(p)=0$ if $\left.\xi\right|_{\mathcal{O}_{K, p}^{\times}} \neq 1$. We follow a similar convention in the later discussions.)

(ii) If $p$ ramifies in $K / \mathbb{Q}$, put

$$
L_{p}(F, \xi ; s)=L_{p}(\xi ; s) Q_{p}\left(\xi_{p}(\Pi) p^{-s}\right)^{-1},
$$

where

$$
Q_{p}(X)=1-p^{-1}\left\{\Lambda_{p}\left(\Phi_{1, p}\right)-p+1\right\} X+X^{2} .
$$

(iii) If $p$ splits in $K / \mathbb{Q}$, put

$$
L_{p}(F, \xi ; s)=Q_{1, p}\left(\xi_{p}\left(\Pi_{1}\right) p^{-s}\right)^{-1} Q_{2, p}\left(\xi_{p}\left(\Pi_{2}\right) p^{-s}\right)^{-1},
$$

where

$$
\begin{gathered}
Q_{1, p}(X)=1-p^{-1} \Lambda_{2} X+p^{-1} \epsilon \Lambda_{1} X^{2}-\epsilon X^{3}, \\
Q_{2, p}(X)=1-p^{-1} \Lambda_{1} X+p^{-1} \epsilon^{-1} \Lambda_{2} X^{2}-\epsilon^{-1} X^{3},
\end{gathered}
$$

with $\Lambda_{i}=\Lambda_{p}\left(\Phi_{i, p}\right) \quad(i=1,2)$ and $\epsilon=\Lambda_{p}\left(\Phi_{0, p}^{+}\right)=\Omega_{p}\left(\Pi_{1} / \Pi_{2}\right)$.

\subsection{Automorphic forms on $U(1,1)$}

We next recall the definition of automorphic forms on $H=U(T)$. In what follows, we fix once and for all an element $\chi_{0}$ of $\mathcal{X}$ with $w_{\infty}\left(\chi_{0}\right)=-1$ (cf. $\left.\S 2.5\right)$. For $u=\left(\begin{array}{ll}a & b \\ c & d\end{array}\right) \in \mathcal{U}_{p}^{\prime}$, put

$$
\tilde{\chi}_{0, p}(u)= \begin{cases}\chi_{0, p}(a) & \text { if } \in p \mathcal{O}_{K, p}, \\ \chi_{0, p}(c) & \text { otherwise. }\end{cases}
$$

Note that $c \in \mathcal{O}_{K, p}^{\times}$if $c \notin p \mathcal{O}_{K, p}$.

Lemma 3.2. For any $p<\infty, \widetilde{\chi}_{0, p}$ is a character of $\mathcal{U}_{p}^{\prime}$.

Proof. The lemma is easily verified when $p \mid D$. Suppose that $p \nmid D$. For $u \in \mathcal{U}_{p}^{\prime}=\mathcal{U}_{p}$, there exists a $t_{u} \in \mathcal{O}_{K, p}^{\times}$such that $\operatorname{det} u=t_{u}^{\sigma} / t_{u}$. It is straightforward to see that $\widetilde{\chi}_{0, p}(u)=\chi_{0, p}^{-1}\left(t_{u}\right)$, from which the lemma follows.

Define a character $\widetilde{\chi}_{0}$ of $\mathcal{U}_{f}^{\prime}$ by $\widetilde{\chi}_{0}=\prod_{p<\infty} \widetilde{\chi}_{0, p}$ and put

$$
j^{\prime}\left(h_{\infty}, z\right)=\operatorname{det} h_{\infty}^{-1} \cdot j\left(h_{\infty}, z\right) \quad\left(h_{\infty} \in H_{\infty}, z \in \mathfrak{H}\right) .
$$

Let $A_{l-1}\left(\mathcal{U}_{f}^{\prime}, \tilde{\chi}_{0}\right)$ be the space of smooth functions $f$ on $H_{\mathbb{Q}} \backslash H_{\mathbf{A}}$ satisfying the following three conditions:

(i) $f\left(h u_{f} u_{\infty}\right)=j^{\prime}\left(u_{\infty}, z_{0}\right)^{1-l} \widetilde{\chi}_{0}\left(u_{f}\right) f(h)\left(h \in H_{\mathbf{A}}, u_{f} \in \mathcal{U}_{f}^{\prime}, u_{\infty} \in \mathcal{U}_{\infty}\right)$; 


\section{A. Murase And T. Sugano}

(ii) for any $h_{f} \in H_{\mathbf{A}, f}, h_{\infty}\left\langle z_{0}\right\rangle \mapsto j^{\prime}\left(h_{\infty}, z_{0}\right)^{l-1} f\left(h_{f} h_{\infty}\right)$ is holomorphic on $\mathfrak{H}$;

(iii) $f$ is holomorphic at each cusp.

We denote by $S_{l-1}\left(\mathcal{U}_{f}^{\prime}, \widetilde{\chi}_{0}\right)$ the space of $f \in A_{l-1}\left(\mathcal{U}_{f}^{\prime}, \widetilde{\chi}_{0}\right)$ satisfying

$$
\int_{\mathbb{Q} \backslash \mathbb{Q}_{\mathbf{A}}} f\left(\mathbf{n}_{H}(x) h\right) d x=0 \quad\left(h \in H_{\mathbf{A}}\right) .
$$

We call $A_{l-1}\left(\mathcal{U}_{f}^{\prime}, \widetilde{\chi}_{0}\right)$ (respectively $S_{l-1}\left(\mathcal{U}_{f}^{\prime}, \widetilde{\chi}_{0}\right)$ ) the space of holomorphic automorphic forms (respectively holomorphic cusp forms) of weight $l-1$ on $\mathcal{U}_{f}^{\prime}$ of character $\tilde{\chi}_{0}$. It is known that $f \in S_{l-1}\left(\mathcal{U}_{f}^{\prime}, \widetilde{\chi}_{0}\right)$ is bounded on $H_{\mathbf{A}}$.

As in $\S 3.1$, we have $S_{l-1}\left(\mathcal{U}_{f}^{\prime}, \tilde{\chi}_{0}\right)=\bigoplus_{\Omega \in \mathcal{Y}_{l}} S_{l-1}\left(\mathcal{U}_{f}^{\prime}, \tilde{\chi}_{0} ; \chi_{0} \Omega\right)$, where $S_{l-1}\left(\mathcal{U}_{f}^{\prime}, \tilde{\chi}_{0} ; \chi_{0} \Omega\right)$ is the space of $f \in S_{l-1}\left(\mathcal{U}_{f}^{\prime}, \tilde{\chi}_{0}\right)$ satisfying $f\left(t 1_{2} \cdot h\right)=\left(\chi_{0} \Omega\right)(t) f(h)$ for $t \in K_{\mathbf{A}}^{1}$.

\subsection{Hecke operators and $L$-functions for $U(1,1)$}

For $p<\infty$, let $\widetilde{\mathcal{H}}_{p}^{H}=\mathcal{H}\left(H_{p}, \mathcal{U}_{p}^{\prime} ; \widetilde{\chi}_{0, p}\right)$ be the space of compactly supported functions $\phi$ on $H_{p}$ satisfying $\phi\left(u_{1} h u_{2}\right)=\widetilde{\chi}_{0, p}\left(u_{1} u_{2}\right) \phi(h)\left(h \in H_{p}, u_{1}, u_{2} \in \mathcal{U}_{p}^{\prime}\right)$. Then $\widetilde{\mathcal{H}}_{p}^{H}$ forms a $\mathbb{C}$-algebra with the product

$$
\left(\phi * \phi^{\prime}\right)(h)=\operatorname{vol}\left(\mathcal{U}_{p}^{\prime}\right)^{-1} \int_{H_{p}} \phi\left(h x^{-1}\right) \phi^{\prime}(x) d x \quad\left(\phi, \phi^{\prime} \in \widetilde{\mathcal{H}}_{p}^{H}, h \in H_{p}\right),
$$

and acts on $S_{l-1}\left(\mathcal{U}_{f}^{\prime}, \tilde{\chi}_{0} ; \chi_{0} \Omega\right)$ by

$$
(f * \phi)(h)=\operatorname{vol}\left(\mathcal{U}_{p}^{\prime}\right)^{-1} \int_{H_{p}} f\left(h x^{-1}\right) \phi(x) d x \quad\left(f \in S_{l-1}\left(\mathcal{U}_{f}^{\prime}, \widetilde{\chi}_{0} ; \chi_{0} \Omega\right), \phi \in \widetilde{\mathcal{H}}_{p}^{H}\right) .
$$

First suppose that $p$ is inert in $K / \mathbb{Q}$. Then $\widetilde{\mathcal{H}}_{p}^{H}=\mathbb{C}\left[\phi_{1, p}\right]$, where $\phi_{1, p}$ is the element of $\widetilde{\mathcal{H}}_{p}^{H}$ satisfying $\operatorname{Supp} \phi_{1, p} \subset \mathcal{U}_{p}^{\prime} \mathbf{d}_{H}(p) \mathcal{U}_{p}^{\prime}$ and $\phi_{1, p}\left(\mathbf{d}_{H}(p)\right)=\chi_{0, p}^{-1}(p)=-1$.

Next suppose that $p$ splits in $K / \mathbb{Q}$. Then $\widetilde{\mathcal{H}}_{p}^{H}=\mathbb{C}\left[\phi_{1, p}, \phi_{2, p}, \phi_{0, p}^{ \pm}\right]$, where $\phi_{1, p}, \phi_{2, p}, \phi_{0, p}^{ \pm}$are the elements of $\widetilde{\mathcal{H}}_{p}^{H}$ satisfying $\operatorname{Supp} \phi_{i, p} \subset \mathcal{U}_{p}^{\prime} \mathbf{d}_{H}\left(\Pi_{i}\right) \mathcal{U}_{p}^{\prime}, \phi_{i, p}\left(\mathbf{d}_{H}\left(\Pi_{i}\right)\right)=\chi_{0, p}^{-1}\left(\Pi_{i}\right)$ and $\operatorname{Supp} \phi_{0, p}^{ \pm} \subset$ $\mathcal{U}_{p}^{\prime} \mathbf{d}_{H}\left(\left(\Pi_{1} / \Pi_{2}\right)^{ \pm 1}\right) \mathcal{U}_{p}^{\prime}=\mathcal{U}_{p}^{\prime}\left(\Pi_{1} / \Pi_{2}\right)^{\mp 1} 1_{2}, \phi_{0, p}^{ \pm}\left(\mathbf{d}_{H}\left(\left(\Pi_{1} / \Pi_{2}\right)^{ \pm 1}\right)\right)=\chi_{0, p}^{-1}\left(\left(\Pi_{1} / \Pi_{2}\right)^{ \pm 1}\right)$. We note that $\phi_{1, p} * \phi_{0, p}^{-}=\phi_{2, p}$. In both cases, $\widetilde{\mathcal{H}}_{p}^{H}$ is commutative and we set $\mathcal{H}_{p}^{H}=\widetilde{\mathcal{H}}_{p}^{H}$.

Finally, suppose that $p$ ramifies in $K / \mathbb{Q}$. In this case, $\widetilde{\mathcal{H}}_{p}^{H}$ is not commutative in general. Let $\phi_{p}^{ \pm}$ be the elements of $\widetilde{\mathcal{H}}_{p}^{H}$ satisfying $\operatorname{Supp} \phi_{p}^{ \pm} \subset \mathcal{U}_{p}^{\prime} \mathbf{d}_{H}\left(\Pi^{ \pm 1}\right) \mathcal{U}_{p}^{\prime}$ and $\phi_{p}^{ \pm}\left(\mathbf{d}_{H}\left(\Pi^{ \pm 1}\right)\right)=\chi_{0, p}^{-1}\left(\Pi^{ \pm 1}\right)$. We let $\mathcal{H}_{p}^{H}$ the subalgebra of $\widetilde{\mathcal{H}}_{p}^{H}$ generated by $\phi_{p}^{+}+\phi_{p}^{-}$. Then $\mathcal{H}_{p}^{H}$ is commutative.

Lemma 3.3. Let $f \in S_{l-1}\left(\mathcal{U}_{f}^{\prime}, \tilde{\chi}_{0} ; \chi_{0} \Omega\right)$.

(i) Suppose that $p$ is inert in $K / \mathbb{Q}$. Then we have

$$
\left(f * \phi_{1, p}\right)(h)=-f\left(h \mathbf{d}_{H}\left(p^{-1}\right)\right)-\sum_{x \in\left(\mathbb{Z}_{p}-p \mathbb{Z}_{p}\right) / p \mathbb{Z}_{p}} f\left(h \mathbf{n}_{H}\left(p^{-1} x\right)\right)-\sum_{x \in \mathbb{Z}_{p} / p^{2} \mathbb{Z}_{p}} f\left(h \mathbf{n}_{H}(x) \mathbf{d}_{H}(p)\right) .
$$

(ii) Suppose that $p$ ramifies in $K / \mathbb{Q}$. Then

$$
\left(f *\left(\phi_{p}^{+}+\phi_{p}^{-}\right)\right)(h)=\chi_{0, p}^{-1}(\Pi) \sum_{x \in \mathbb{Z}_{p} / p \mathbb{Z}_{p}} f\left(h \overline{\mathbf{n}}_{H}(D x) \mathbf{d}_{H}\left(\Pi^{-1}\right)\right)+\chi_{0, p}(\Pi) \sum_{x \in \mathbb{Z}_{p} / p \mathbb{Z}_{p}} f\left(h \mathbf{n}_{H}(x) \mathbf{d}_{H}(\Pi)\right) .
$$




\section{ON THE Fourier-JACOBI EXPANSION OF THE UNITARY KUDLA LIFT}

(iii) Suppose that $p$ splits in $K / \mathbb{Q}$. Then we have

$$
\begin{aligned}
& \left(f * \phi_{1, p}\right)(h)=\chi_{0, p}^{-1}\left(\Pi_{1}\right)\left\{f\left(h \mathbf{d}_{H}\left(\Pi_{1}^{-1}\right)\right)+\sum_{x \in \mathbb{Z}_{p} / p \mathbb{Z}_{p}} f\left(h \mathbf{n}_{H}(x) \mathbf{d}_{H}\left(\Pi_{2}\right)\right)\right\}, \\
& \left(f * \phi_{2, p}\right)(h)=\chi_{0, p}^{-1}\left(\Pi_{2}\right)\left\{f\left(h \mathbf{d}_{H}\left(\Pi_{2}^{-1}\right)\right)+\sum_{x \in \mathbb{Z}_{p} / p \mathbb{Z}_{p}} f\left(h \mathbf{n}_{H}(x) \mathbf{d}_{H}\left(\Pi_{1}\right)\right)\right\}, \\
& \left(f * \phi_{0, p}^{ \pm}\right)(h)=\chi_{0, p}^{-1}\left(\left(\Pi_{1} / \Pi_{2}\right)^{ \pm 1}\right) \cdot f\left(h \mathbf{d}_{H}\left(\Pi_{1} / \Pi_{2}\right)^{\mp 1}\right) \quad\left(=\Omega_{p}^{ \pm 1}\left(\Pi_{1} / \Pi_{2}\right) f(h)\right) .
\end{aligned}
$$

If, for every $p<\infty$, there exists a $\mathbb{C}$-algebra homomorphism $\lambda_{p}$ of $\mathcal{H}_{p}^{H}$ to $\mathbb{C}$ such that $f * \phi=$ $\lambda_{p}(\phi) f$ holds for any $\phi \in \mathcal{H}_{p}^{H}$, we say that $f$ is a Hecke eigenform with eigenvalues $\left\{\lambda_{p}\right\}$.

Let $f \in S_{l-1}\left(\mathcal{U}_{f}^{\prime}, \widetilde{\chi}_{0} ; \chi_{0} \Omega\right)$ be a Hecke eigenform with eigenvalues $\left\{\lambda_{p}\right\}$ and $\xi$ a Hecke character of $K$. We define an $L$-function

$$
L(f, \xi ; s)=\prod_{p<\infty} L_{p}(f, \xi ; s),
$$

where the local factor $L_{p}(f, \xi ; s)$ is given as follows.

(i) If $p$ is inert in $K / \mathbb{Q}$, put

$$
L_{p}(f, \xi ; s)=R_{p}\left(\xi_{p}(p) p^{-2 s}\right)^{-1}
$$

where

$$
R_{p}(X)=1-\left\{p^{-1} \lambda_{p}\left(\phi_{1, p}\right)+1-p^{-1}\right\} X+X^{2} .
$$

(ii) If $p$ ramifies in $K / \mathbb{Q}$, put

$$
L_{p}(f, \xi ; s)=R_{p}\left(\xi_{p}(\Pi) p^{-s}\right)^{-1}
$$

where

$$
R_{p}(X)=1-p^{-1 / 2} \lambda_{p}\left(\phi_{p}^{+}+\phi_{p}^{-}\right) X+X^{2} .
$$

(iii) If $p$ splits in $K / \mathbb{Q}$, put

$$
L_{p}(f, \xi ; s)=R_{1, p}\left(\xi_{p}\left(\Pi_{1}\right) p^{-s}\right)^{-1} R_{2}\left(\xi_{p}\left(\Pi_{2}\right) p^{-s}\right)^{-1},
$$

where

$$
R_{1, p}(X)=1-p^{-1 / 2} \lambda_{p}\left(\phi_{1, p}\right) X+\epsilon X^{2}, \quad R_{2, p}(X)=1-p^{-1 / 2} \lambda_{p}\left(\phi_{2, p}\right) X+\epsilon^{-1} X^{2}
$$

with $\epsilon=\lambda_{p}\left(\phi_{0, p}^{+}\right)=\Omega_{p}\left(\Pi_{1} / \Pi_{2}\right)$. Note that $\lambda_{p}\left(\phi_{2, p}\right)=\epsilon^{-1} \lambda_{p}\left(\phi_{1, p}\right)$ and, hence, $R_{2, p}(X)=$ $R_{1, p}\left(\epsilon^{-1} X\right)$.

Remark. Suppose that $K$ has class number 1. For $f \in S_{l-1}\left(\mathcal{U}_{f}^{\prime}, \tilde{\chi}_{0}\right)$, put $f_{d m}\left(h_{\infty}\langle i\rangle\right)=$ $j^{\prime}\left(h_{\infty}, i\right)^{l-1} f\left(h_{\infty}\right)\left(h_{\infty} \in H_{\infty}\right)$. Then $f \mapsto f_{d m}$ gives rise to an isomorphism between $S_{l-1}\left(\mathcal{U}_{f}^{\prime}, \widetilde{\chi}_{0}\right)$ and the space of holomorphic cusp forms of weight $l-1$ and character $\omega$ on $\Gamma_{0}(D)$. Assume that $f_{d m}(z)=\sum_{n=0}^{\infty} c(n) \mathbf{e}[n z]$ is a normalized newform in the sense of [Li75]. Define a twisted $L$-function

$$
Z\left(f_{d m} ; s\right)=\sum_{\mathfrak{a}} c(\mathrm{Na}) \alpha^{l} \mathrm{Na}^{-s},
$$

$\mathfrak{a}=\alpha \mathcal{O}_{K}$ running over the nonzero integral ideals of $K$. A straightforward calculation shows that

$$
L(f, \mathbf{1} ; s)=\zeta(2 s) Z\left(f_{d m} ; s+l-1\right) \prod_{p \mid D}\left(1-p^{-2 s}\right)\left(1-\overline{c(p) \eta_{p}^{l}} p^{-s-l+1}\right)^{-1} .
$$

Here, for $p \mid D, \eta_{p}$ is an element of $K$ such that $\eta_{p} \mathcal{O}_{K}$ is a prime ideal of $K$ dividing $p$. 


\section{A. Murase and T. Sugano}

\section{Theta functions}

\subsection{The space of theta functions}

In this section, we recall several facts about theta functions on $R$ (for details, see [MS02, §2]). For $m \in \mathbb{Q}^{\times}$, let $\mathbf{T}^{m}$ be the space of smooth functions $\Theta$ on $R_{\mathbb{Q}} \backslash R_{\mathbf{A}}$ satisfying:

(i) $\Theta((0, x) r)=\psi_{m}(x) \Theta(r)\left(x \in \mathbb{Q}_{\mathbf{A}}, r \in R_{\mathbf{A}}\right)$;

(ii) for any $r \in R_{\mathbf{A}}, t_{\infty} \mapsto \Theta\left(r t_{\infty}\right)$ is $K_{\infty}^{1}$-finite.

Let $\rho^{\prime}$ be the smooth representation of $R_{\mathbf{A}, f}$ on $\mathbf{T}^{m}$ given by right translations. Define an inner product $\langle\langle\cdot, \cdot\rangle\rangle_{R}$ on $\mathbf{T}^{m}$ by

$$
\left\langle\left\langle\Theta, \Theta^{\prime}\right\rangle\right\rangle_{R}=\int_{R_{\mathbb{Q}} \backslash R_{\mathbf{A}}} \Theta(r) \overline{\Theta^{\prime}(r)} d r .
$$

\subsection{Lattice model}

In this and the following subsections, we recall the definition of metaplectic representations on a lattice model (for the details, see [MVW87, ch. 2, I. 4], [MS00, §1] and [MS02, § 2]). Let $m \in \mathbb{Q}^{\times}$ and $\mathcal{L}=(m D)^{-1} \mathbb{Z}+2^{-1} \kappa \mathbb{Z}$ be a lattice in $K$. For a rational prime $p$, let $V_{p}^{m}$ be the space of $\Phi_{p} \in \mathcal{S}\left(K_{p}\right)$ satisfying

$$
\Phi_{p}\left(z_{p}+l_{p}\right)=\psi_{m, p}\left(\frac{1}{2}\left\langle z_{p}, l_{p}\right\rangle_{\kappa}+\frac{1}{4}\left\langle l_{p}, l_{p}^{\sigma}\right\rangle_{\kappa}\right) \Phi_{p}\left(z_{p}\right)
$$

for $z_{p} \in K_{p}$ and $l_{p} \in \mathcal{L}_{p}=\mathcal{L} \otimes_{\mathbb{Z}} \mathbb{Z}_{p}$. Let $V_{\infty}^{m}$ be the space consisting of functions on $K_{\infty}=\mathbb{C}$ of the form $\overline{P\left(z_{\infty}\right)} \mathbf{e}\left[2^{-1} m \kappa z_{\infty} \overline{z_{\infty}}\right]$ (respectively $\left.P\left(z_{\infty}\right) \mathbf{e}\left[-2^{-1} m \kappa z_{\infty} \overline{z_{\infty}}\right]\right)$ if $m>0$ (respectively if $m<0$ ), where $P$ is a polynomial in $z_{\infty}$. Define an inner product $(\cdot, \cdot)_{v}$ on $V_{v}^{m}$ by

$$
\left(\Phi_{v}, \Phi_{v}^{\prime}\right)_{v}=\int_{K_{v}} \Phi_{v}\left(z_{v}\right) \overline{\Phi_{v}^{\prime}\left(z_{v}\right)} d^{m} z_{v} \quad\left(\Phi_{v}, \Phi_{v}^{\prime} \in V_{v}^{m}\right),
$$

where $d^{m} z_{v}$ is the Haar measure on $K_{v}$ self-dual with respect to the pairing $(z, w) \mapsto \psi_{m, v}\left(\langle z, w\rangle_{\kappa}\right)$ (note that $\left.d^{m} z_{v}=|\mathrm{N}(m \kappa)|_{v}^{1 / 2} d z_{v}\right)$.

Let $V^{m}$ be the restricted tensor product of $V_{v}^{m}$ over primes $v$ of $\mathbb{Q}$ with respect to $\left\{\Phi_{0, p}\right\}$, where $\Phi_{0, p} \in V_{p}^{m}$ is given by

$$
\Phi_{0, p}\left(z_{p}\right)=\operatorname{char}_{\mathcal{L}_{p}}\left(z_{p}\right) \psi_{m, p}\left(\frac{1}{4}\left\langle z_{p}, z_{p}^{\sigma}\right\rangle_{\kappa}\right) \quad\left(z_{p} \in K_{p}\right)
$$

We denote by $V_{L^{2}}^{m}$ the completion of $V^{m}$ with respect to the inner product

$$
\left(\Phi, \Phi^{\prime}\right)=\int_{K_{\mathbf{A}}} \Phi(z) \overline{\Phi^{\prime}(z)} d^{m} z
$$

where $d^{m} z=\prod_{v} d^{m} z_{v}$. Let $\rho^{m}$ be a unitary representation of $N_{\mathbf{A}}$ on $V_{L^{2}}^{m}$ defined by

$$
\rho^{m}(w, x) \Phi(z)=\psi_{m}\left(\frac{1}{2}\langle z, w\rangle_{\kappa}+x\right) \Phi(z+w) \quad\left(\Phi \in V_{L^{2}}^{m},(w, x) \in N_{\mathbf{A}}, z \in K_{\mathbf{A}}\right) .
$$

Then $\rho^{m}$ is irreducible.

\subsection{Metaplectic representation of $K_{\mathrm{A}}^{\mathbf{1}}$}

Let $\chi \in \mathcal{X}$ and $v$ be a prime of $\mathbb{Q}$. Let $\lambda_{K_{v}}\left(\psi_{m, v}\right)$ be the Weil constant attached to $\left(K_{v} / \mathbb{Q}_{v}, \psi_{m, v}\right)$ (cf. [Wei64, Théorèm 2]). By definition, we have

$$
\int_{K_{v}} \varphi(z) \psi_{v}\left(m z z^{\sigma}\right) d z=\lambda_{K_{v}}\left(\psi_{m, v}\right)|m|_{v}^{-1} \int_{K_{v}} \widehat{\varphi}(z) \psi_{v}\left(-m^{-1} z z^{\sigma}\right) d z
$$

for $\varphi \in \mathcal{S}\left(K_{v}\right)$. Here the Fourier transform $\widehat{\varphi}$ of $\varphi$ is defined by

$$
\widehat{\varphi}(z)=\int_{K_{v}} \varphi(w) \psi_{v}\left(\operatorname{Tr}\left(w^{\sigma} z\right)\right) d w \quad\left(z \in K_{v}\right) .
$$




\section{ON THE Fourier-JACOBI EXPANSION OF THE UNITARY KUDLA LIFT}

For $t_{v} \in K_{v}^{1}$, we define an endomorphism $\mathcal{M}_{\chi}^{\kappa, m}\left(t_{v}\right)$ of $V_{L^{2}}^{m}$ as follows. If $t_{v}=1$, we put $\mathcal{M}_{\chi}^{\kappa, m}\left(t_{v}\right)=$ $\operatorname{Id}_{V_{L^{2}}^{m}}$. Suppose that $t_{v} \neq 1$. For $\Phi \in V^{m}$, we put

$$
\begin{aligned}
\mathcal{M}_{\chi}^{\kappa, m}\left(t_{v}\right) \Phi(z)= & \lambda_{K_{v}}\left(\psi_{m, v}\right)^{-1} \chi_{v}\left(\frac{1-t_{v}}{\kappa}\right)\left|\mathrm{N}\left(1-t_{v}\right)\right|_{v}^{-1 / 2} \\
& \times \int_{K_{v}} \psi_{m, v}\left(\frac{1}{2} \operatorname{Tr}\left(\frac{\kappa}{1-t_{v}}\right) w_{v} w_{v}^{\sigma}\right) \rho^{m}\left(w_{v}, 0\right) \Phi(z) d^{m} w_{v} \quad\left(z \in K_{\mathbf{A}}\right) .
\end{aligned}
$$

It is known that $\mathcal{M}_{\chi}^{\kappa, m}\left(t_{v}\right)$ preserves $V^{m}$ and extends uniquely to a unitary operator of $V_{L^{2}}^{m}$. For $t=\left(t_{v}\right)_{v} \in K_{\mathbf{A}}^{1}$, we put $\mathcal{M}_{\chi}^{\kappa, m}(t)=\bigotimes_{v} \mathcal{M}_{\chi}^{\kappa, m}\left(t_{v}\right)$. Then $\mathcal{M}_{\chi}^{\kappa, m}$ defines a unitary representation of $K_{\mathbf{A}}^{1}$ on $V_{L^{2}}^{m}$ satisfying $\mathcal{M}_{\chi}^{\kappa, m}(t) \rho^{m}(w, x) \mathcal{M}_{\chi}^{\kappa, m}\left(t^{-1}\right)=\rho^{m}(t w, x)\left(t \in K_{\mathbf{A}}^{1},(w, x) \in N_{\mathbf{A}}\right)$. Hence, we can extend $\mathcal{M}_{\chi}^{\kappa, m}$ to a unitary representation of $R_{\mathbf{A}}$ on $V_{L^{2}}^{m}$ by

$$
\mathcal{M}_{\chi}^{\kappa, m}((w, x) t)=\rho^{m}(w, x) \circ \mathcal{M}_{\chi}^{\kappa, m}(t) \quad\left((w, x) \in N_{\mathbf{A}}, t \in K_{\mathbf{A}}^{1}\right) .
$$

\subsection{Theta series}

For $\chi \in \mathcal{X}$ and $\Phi \in V^{m}$, we set

$$
\theta_{\chi, \Phi}^{m}(r)=\sum_{X \in K}\left(\mathcal{M}_{\chi}^{\kappa, m}(r) \Phi\right)(X) \quad\left(r \in R_{\mathbf{A}}\right) .
$$

The theta series $\theta_{\chi, \Phi}^{m}$ is absolutely convergent and belongs to $\mathbf{T}^{m}$. Let $\mathbf{T}_{\chi}^{m}=\left\{\theta_{\chi, \Phi}^{m} \mid \Phi \in V^{m}\right\}$.

Proposition 4.1 ([Shi79, Proposition 2]; see also [MS02, Theorem 2.22]). We have the following.

(i) We have an orthogonal decomposition

$$
\mathbf{T}^{m}=\bigoplus_{\chi \in \mathcal{X}} \mathbf{T}_{\chi}^{m}
$$

(ii) For $\chi \in \mathcal{X}$ and $\Phi, \Phi^{\prime} \in V^{m}$, we have $\left\langle\left\langle\theta_{\chi, \Phi}^{m}, \theta_{\chi, \Phi^{\prime}}^{m}\right\rangle\right\rangle_{R}=c_{m}\left(\Phi, \Phi^{\prime}\right)$ with a positive constant $c_{m}$ depending only on $m$.

\subsection{Holomorphic theta functions}

For $k \in \mathbb{Z}$, set $\mathbf{T}^{m, k}=\left\{\Theta \in \mathbf{T}^{m} \mid \Theta\left(r t_{\infty}\right)=t_{\infty}^{k} \Theta(r)\left(r \in R_{\mathbf{A}}, t_{\infty} \in K_{\infty}^{1}\right)\right\}$ and $\mathbf{T}_{\chi}^{m, k}=\mathbf{T}_{\chi}^{m} \cap \mathbf{T}^{m, k}$. Let $\mathbf{T}_{\text {hol }}^{m}$ be the space of $\Theta \in \mathbf{T}^{m, 0}$ such that

$$
w_{\infty} \mapsto f_{\Theta, r_{f}}\left(w_{\infty}\right):=\mathbf{e}_{m}\left[-\frac{\kappa}{2} w_{\infty} \overline{w_{\infty}}\right] \Theta\left(\left(w_{\infty}, 0\right) r_{f}\right)
$$

is holomorphic on $\mathbb{C}$ for any $r_{f} \in R_{\mathbf{A}, f}$. We call $\mathbf{T}_{\text {hol }}^{m}$ the space of holomorphic theta functions.

Lemma 4.2. We have the following.

(i) We have

$$
\mathbf{T}_{\chi}^{m}=\left\{\begin{array}{cl}
\bigoplus_{k \geqslant\left(w_{\infty}(\chi)+1\right) / 2} \mathbf{T}_{\chi}^{m, k} & \text { if } m>0 \\
\bigoplus_{k \leqslant\left(w_{\infty}(\chi)-1\right) / 2} \mathbf{T}_{\chi}^{m, k} & \text { if } m<0 .
\end{array}\right.
$$

(ii) We have

$$
\mathbf{T}_{\mathrm{hol}}^{m}= \begin{cases}\bigoplus_{\chi \in \mathcal{X}, w_{\infty}(\chi)=-1} \mathbf{T}_{\chi}^{m, 0} & \text { if } m>0, \\ \{0\} & \text { if } m<0 .\end{cases}
$$




\section{A. Murase and T. Sugano}

Proof. If $m>0$, the assertions (i) and (ii) are direct consequences of Lemma 2.14 and Proposition 2.26 in [MS02]. Suppose that $m<0$. Observe that $\overline{V^{m}}=V^{-m}$ and that $\rho^{m}(w, x) \Phi=$ $\overline{\rho^{-m}(w, x) \bar{\Phi}}$ for $\Phi \in V^{m}$. This implies that $\mathcal{M}_{\chi}^{\kappa, m}(r) \Phi=\overline{\mathcal{M}_{\chi^{-1}}^{\kappa,-m}(r) \bar{\Phi}}\left(r \in R_{\mathbf{A}}\right)$ and, hence, $\theta_{\chi, \Phi}^{m}=\overline{\theta_{\chi^{-1}, \Phi}^{-m}}$. It follows that

$$
\mathbf{T}_{\chi}^{m}=\overline{\mathbf{T}_{\chi^{-1}}^{-m}}=\bigoplus_{k \geqslant\left(-w_{\infty}(\chi)+1\right) / 2} \overline{\mathbf{T}_{\chi^{-1}}^{-m, k}}=\bigoplus_{k \leqslant\left(w_{\infty}(\chi)-1\right) / 2} \mathbf{T}_{\chi}^{m, k},
$$

which proves assertion (i). To show assertion (ii), let $\Theta \in \mathbf{T}_{\text {hol }}^{m}$ and $r_{f} \in R_{\mathbf{A}, f}$. It is easily verified that $f_{\Theta, r_{f}}\left(w_{\infty}\right)$ is holomorphic, bounded on $\mathbb{C}$ and tends to zero when $\left|w_{\infty}\right| \rightarrow \infty$. This implies that $f_{\Theta, r_{f}}\left(w_{\infty}\right)$ is identically equal to zero and, hence, $\Theta=0$.

\subsection{Primitive theta functions}

Let $\mathfrak{a}$ be an ideal of $K$. Define an open compact subgroup $N(\mathfrak{a})_{f}$ of $N_{\mathbf{A}, f}$ by

$$
\begin{aligned}
N(\mathfrak{a})_{f} & =\left\{(w, x) \in N_{\mathbf{A}, f} \mid w \in \mathfrak{a}_{f}, x+\frac{\kappa}{2} w w^{\sigma} \in \mathfrak{a}_{f} \mathfrak{a}_{f}^{\sigma}\right\} \\
& =\left\{\left(w, x+x_{w}\right) \in N_{\mathbf{A}, f} \mid w \in \mathfrak{a}_{f}, x \in \mathfrak{a}_{f} \mathfrak{a}_{f}^{\sigma}\right\}
\end{aligned}
$$

(recall that $\left.x_{w}=2^{-1} \operatorname{Tr}(\theta) w w^{\sigma}\right)$. We put $R(\mathfrak{a})_{f}=\left\{n t \mid n \in N(\mathfrak{a})_{f}, t \in \mathcal{O}_{K, f}^{1}\right\}$. Let $\mathbf{T}^{m}(\mathfrak{a})=$ $\left\{\Theta \in \mathbf{T}^{m} \mid \rho^{\prime}\left(r_{0}\right) \Theta=\Theta\right.$ for any $\left.r_{0} \in R(\mathfrak{a})_{f}\right\}$ and $\mathbf{T}_{\text {hol }}^{m}(\mathfrak{a})=\mathbf{T}_{\text {hol }}^{m} \cap \mathbf{T}^{m}(\mathfrak{a})$. It is easy to see that $\mathbf{T}_{\text {hol }}^{m}(\mathfrak{a})=\{0\}$ unless $m>0$ and $m \mathrm{~N}(\mathfrak{a})$ is integral. We henceforth assume that $m>0$ and $m \mathrm{~N}(\mathfrak{a})$ is integral. Then

$$
\mathbf{T}_{\text {hol }}^{m}(\mathfrak{a})=\bigoplus_{\chi \in \mathcal{X}, w_{\infty}(\chi)=-1} \mathbf{T}_{\text {hol }}^{m}(\mathfrak{a}, \chi),
$$

where $\mathbf{T}_{\text {hol }}^{m}(\mathfrak{a}, \chi)=\mathbf{T}_{\text {hol }}^{m}(\mathfrak{a}) \cap \mathbf{T}_{\chi}^{m}$.

To define the primitive part of $\mathbf{T}_{\text {hol }}^{m}(\mathfrak{a})$, let $\mathfrak{b}$ be an ideal of $K$ with $\mathfrak{a} \subset \mathfrak{b}$. Define an endomorphism $\mathcal{P}_{\mathfrak{b}}^{\prime}$ of $\mathbf{T}_{\mathrm{hol}}^{m}(\mathfrak{a})$ by

$$
\mathcal{P}_{\mathfrak{b}}^{\prime} \Theta=\int_{N(\mathfrak{b})_{f}} \rho^{\prime}\left(n_{f}\right) \Theta d_{\mathfrak{b}} n_{f} \quad\left(\Theta \in \mathbf{T}_{\mathrm{hol}}^{m}(\mathfrak{a})\right)
$$

where $d_{\mathfrak{b}} n_{f}$ is the Haar measure on $N_{\mathbf{A}, f}$ normalized by $\operatorname{vol}\left(N(\mathfrak{b})_{f}\right)=1$. Note that $\mathcal{P}_{\mathfrak{b}}^{\prime}=0$ unless $m \mathrm{~N}(\mathfrak{b})$ is integral. Let

$$
\mathbf{T}_{\text {hol,prim }}^{m}(\mathfrak{a})=\left\{\Theta \in \mathbf{T}_{\text {hol }}^{m}(\mathfrak{a}) \mid \mathcal{P}_{\mathfrak{b}}^{\prime} \Theta=0 \text { for any ideal } \mathfrak{b} \text { of } K \text { with } \mathfrak{b} \supsetneqq \mathfrak{a}\right\}
$$

and

$$
\mathbf{T}_{\text {hol,prim }}^{m}(\mathfrak{a}, \chi)=\mathbf{T}_{\text {hol,prim }}^{m}(\mathfrak{a}) \cap \mathbf{T}_{\chi}^{m} .
$$

We call $\mathbf{T}_{\text {hol,prim }}^{m}(\mathfrak{a}, \chi)$ the space of holomorphic primitive theta functions with respect to (a, $\chi$ ).

Theorem 4.3 (Shintani [Shi79, §2.11] and Glaubermann-Rogawski [GR89, Theorem 4.2]; see also [MS00, Corollary 6.5] and [MS02, Theorem 3.4]). The space $\mathbf{T}_{\text {hol,prim }}^{m}(\mathfrak{a}, \chi)$ is at most onedimensional.

\subsection{Artin conductor and epsilon factor}

Let $p<\infty$ and $m \in \mathbb{Q}_{p}^{\times}$. For $\chi \in \mathcal{X}_{p}$, we put $A_{p}(\chi)=\operatorname{Min}\left\{a \geqslant 0|\chi|_{\left(1+\mathfrak{P}_{p}^{a}\right) \cap \mathcal{O}_{K, p}^{\times}}=1\right\}$, where

$$
\mathfrak{P}_{p}= \begin{cases}p \mathcal{O}_{K, p} & \text { if } p \text { splits in } K / \mathbb{Q} \\ \text { the maximal ideal of } \mathcal{O}_{K, p} & \text { otherwise. }\end{cases}
$$




\section{ON THE Fourier-JACOBI EXPANSION OF THE UNITARY KUDLA LIFT}

Let $\epsilon_{p}\left(s, \chi, \psi_{m, K_{p}}\right)$ be Tate's epsilon factor [Tat79, $\left.\S 3\right]$, where $\psi_{m, K_{p}}=\psi_{m} \circ \operatorname{Tr}_{K_{p} / \mathbb{Q}_{p}}$. It is known that $\epsilon_{p}\left(1 / 2, \chi, \psi_{m, K_{p}}\right)= \pm \chi\left(\kappa^{-1}\right)$. Let $\mathfrak{a}$ be an ideal of $K_{p}$ such that $\mu_{p}(m, \mathfrak{a}):=\operatorname{ord}_{p} m \mathrm{~N}(\mathfrak{a}) \geqslant 0$. Denote by $\mathcal{X}_{p, \operatorname{prim}}(m, \mathfrak{a})$ the set of $\chi \in \mathcal{X}_{p}$ which satisfies

$$
A_{p}(\chi)= \begin{cases}\mu_{p}(m, \mathfrak{a}) & \text { if } \delta_{p}=0 \\ 2\left(\mu_{p}(m, \mathfrak{a})+\delta_{p}\right) & \text { if } \delta_{p}>0 \text { and } \mu_{p}(m, \mathfrak{a})>0 \\ 2 \delta_{p} \text { or } 2 \delta_{p}-1 & \text { if } \delta_{p}>0 \text { and } \mu_{p}(m, \mathfrak{a})=0 .\end{cases}
$$

where $\delta_{p}=\operatorname{ord}_{p} D$.

\subsection{Epsilon dichotomy}

Going back to the global situation, we let $m \in \mathbb{Q}^{\times}, \mathfrak{a}$ an ideal of $K$ and $\chi \in \mathcal{X}$ with $w_{\infty}(\chi)=-1$. Assume that $m>0$ and $m \mathrm{~N}(\mathfrak{a})$ is integral. For $p<\infty$, we write $\chi_{p}$ for the $p$-component of $\chi$. Set

$$
\mathcal{X}_{\text {prim }}(m, \mathfrak{a})=\left\{\chi \in \mathcal{X} \mid w_{\infty}(\chi)=-1, \chi_{p} \in \mathcal{X}_{p, \operatorname{prim}}\left(m, \mathfrak{a}_{p}\right) \text { for every } p<\infty\right\}
$$

and

$$
\mathcal{X}_{\text {prim }}^{+}(m, \mathfrak{a})=\left\{\chi \in \mathcal{X}_{\text {prim }}(m, \mathfrak{a}) \mid \epsilon_{p}\left(1 / 2, \chi_{p}, \psi_{m, K_{p}}\right)=\chi_{p}\left(\kappa^{-1}\right) \text { for every } p<\infty\right\} .
$$

The following result gives a criterion for the existence of primitive theta functions.

Theorem 4.4 ([MS00, Corollary 6.7] and [MS02, Theorem 3.8]). For $\chi \in \mathcal{X}$, the space $\mathbf{T}_{\text {hol,prim }}^{m}(\mathfrak{a}, \chi)$ defined in $\S 4.6$ is one-dimensional if and only if $\chi \in \mathcal{X}_{\text {prim }}^{+}(m, \mathfrak{a})$.

Remark. Theorem 4.4 is a refined form of 'epsilon dichotomy' for $U(1)$ (for epsilon dichotomy for unitary groups, we refer to [Moe91], [Rog92] and [HKS96]).

Let $\mathcal{E}$ be the set of the triplets $(m, \mathfrak{a}, \chi)$, where $m$ is a positive rational number, $\mathfrak{a}$ an ideal of $K$ with $m \mathrm{~N}(\mathfrak{a}) \in \mathbb{Z}$ and $\chi \in \mathcal{X}_{\text {prim }}^{+}(m, \mathfrak{a})$. By Theorem 4.4, we have $\operatorname{dim} \mathbf{T}_{\text {hol,prim }}^{m}(\mathfrak{a}, \chi)=1$ for $(m, \mathfrak{a}, \chi) \in \mathcal{E}$

\subsection{The structure of $T_{\text {hol }}^{m}(\mathfrak{a})$}

We keep the notation and assumptions of $\S 4.8$.

Theorem 4.5 ([Shi79, Proposition 2]; see also [MS02, Theorem 3.9]). We have a direct sum decomposition:

$$
\mathbf{T}_{\text {hol }}^{m}(\mathfrak{a})=\sum_{\mathfrak{a}^{\prime} \in \mathcal{R}(\mathfrak{a})} \sum_{\chi \in \mathcal{X}_{\text {prim }}^{+}\left(m, \mathfrak{a}^{\prime}\right)} \mathbf{T}_{\text {hol,prim }}^{m}\left(\mathfrak{a}^{\prime}, \chi\right),
$$

where $\mathcal{R}(\mathfrak{a})$ is the set of ideals $\mathfrak{a}^{\prime}$ of $K$ containing $\mathfrak{a}$ with $m \mathrm{~N}\left(\mathfrak{a}^{\prime}\right)$ integral.

Remark. Let $\mathfrak{a}_{1}, \mathfrak{a}_{2} \in \mathcal{R}(\mathfrak{a})$ and $\chi_{1} \in \mathcal{X}_{\text {prim }}^{+}\left(m, \mathfrak{a}_{1}\right), \chi_{2} \in \mathcal{X}_{\text {prim }}^{+}\left(m, \mathfrak{a}_{2}\right)$. Then $\mathbf{T}_{\text {hol,prim }}^{m}\left(\mathfrak{a}_{1}, \chi_{1}\right)$ and $\mathbf{T}_{\text {hol,prim }}^{m}\left(\mathfrak{a}_{2}, \chi_{2}\right)$ are orthogonal to each other if at least one of the following conditions is satisfied (see [MS00], §10):

(i) $\chi_{1} \neq \chi_{2}$;

(ii) $m \mathrm{~N}\left(\mathfrak{a}_{1}\right) \neq m \mathrm{~N}\left(\mathfrak{a}_{2}\right)$;

(iii) there exists a prime factor of $m \mathrm{~N}\left(\mathfrak{a}_{1}\right)$ which splits in $K / \mathbb{Q}$.

\subsection{Exceptional case}

Suppose that $p$ ramifies in $K / \mathbb{Q}$ and define an endomorphism $\mathcal{Q}_{p}$ of $\mathbf{T}_{\text {hol }}^{m}(\mathfrak{a})$ by

$$
\mathcal{Q}_{p} \Theta(r)=\int_{\Pi_{p}^{-1} \mathfrak{a}_{p}} \Theta\left(r\left(w, x_{w}\right)\right) d_{\Pi_{p}^{-1} \mathfrak{a}_{p}} w \quad\left(\Theta \in \mathbf{T}_{\text {hol }}^{m}(\mathfrak{a})\right)
$$




\section{A. Murase And T. Sugano}

(for the definition of $d_{\Pi_{p}^{-1} \mathfrak{a}_{p}} w$, see $\S 2.4$ ). Note that $\mathcal{Q}_{p}$ coincides with $\mathcal{P}_{\Pi_{p}^{-1} \mathfrak{a}_{p}}^{\prime}$ if $\mu_{p}(\mathfrak{a}, m)>0$. We say that $\chi_{p} \in \mathcal{X}_{p}$ is ordinary (respectively exceptional) if $A_{p}\left(\chi_{p}\right) \geqslant 2 \delta_{p}$ (respectively $A_{p}\left(\chi_{p}\right)=2 \delta_{p}-1$ ).

Proposition 4.6 [MS02, Theorem 3.8]. Let $\chi \in \mathcal{X}_{\text {prim }}^{+}(m, \mathfrak{a})$ and $\Theta \in \mathbf{T}_{\text {hol,prim }}^{m}(\mathfrak{a}, \chi)$. Suppose that $p$ ramifies in $K / \mathbb{Q}$. Then

$$
\mathcal{Q}_{p} \Theta= \begin{cases}0 & \text { if } \chi_{p} \text { is ordinary } \\ \Theta & \text { if } \chi_{p} \text { is exceptional. }\end{cases}
$$

\subsection{Periods of theta functions}

For $\Theta \in \mathbf{T}^{m}$, we put

$$
I_{\Theta}(r)=\int_{K^{1} \backslash K_{\mathbf{A}}^{1}} \Theta(t r) d^{\times} t \quad\left(r \in R_{\mathbf{A}}\right)
$$

and

$$
I(\Theta)=I_{\Theta}(1)=\int_{K^{1} \backslash K_{\mathbf{A}}^{1}} \Theta(t) d^{\times} t .
$$

We call $I(\Theta)$ the period of $\Theta$. The following fact is proved by Yang [Yan97, Theorem 0.3] (see also [MS02, Theorem 5.2]).

Theorem 4.7. Let $(m, \mathfrak{a}, \chi) \in \mathcal{E}$ and $\Theta \in \mathbf{T}_{\text {hol,prim }}^{m}(\mathfrak{a}, \chi)-\{0\}$.

(i) We have

$$
|I(\Theta)|^{2}=C(\chi) L\left(\chi ; \frac{1}{2}\right)\langle\langle\Theta, \Theta\rangle\rangle_{R}
$$

where

$$
C(\chi)=\frac{1}{2 L(\omega ; 1)} \prod_{p \nmid D, A_{p}\left(\chi_{p}\right)>0}\left(1+\omega_{p}\left(\pi_{p}\right) p^{-1}\right)^{-1}
$$

and $\left\langle\langle,\rangle_{R}\right.$ is defined in $\S 4$ 4. (Recall that $\omega$ is the quadratic Hecke character of $\mathbb{Q}$ associated with the extension $K / \mathbb{Q}, \omega_{p}$ the p-component of $\omega$ and $\pi_{p}$ a prime element of $\mathbb{Q}_{p}$.)

(ii) We have $I(\Theta) \neq 0$ if and only if $L(\chi ; 1 / 2) \neq 0$.

4.12 The following result will be needed in the next section.

Proposition 4.8. Suppose that $p$ splits in $K / \mathbb{Q}$ and $\mu_{p}(m, \mathfrak{a})=0$. Let $\chi \in \mathcal{X}_{\text {prim }}^{+}(m, \mathfrak{a})$ and $\Theta \in \mathbf{T}_{\text {hol,prim }}^{m}(\mathfrak{a}, \chi)$. For $t \in K_{p}^{1}-\mathcal{O}_{K, p}^{1}$, we have

$$
\int_{\mathfrak{a}_{p}} \Theta\left(r\left(w, x_{w}\right) t^{-1}\right) d_{\mathfrak{a}_{p}} w=|\mathrm{N}(1-t)|_{p}^{-1 / 2} \chi_{p}\left(\frac{1-t^{-1}}{\kappa}\right) \Theta(r) \quad\left(r \in R_{\mathbf{A}}\right) .
$$

Proof. First note that we may (and do) let $\theta=(1,0)$ and $x_{w}=\frac{1}{2} \mathrm{~N}(w)$. Take $\Phi^{\prime}=\otimes_{v} \Phi_{v}^{\prime} \in V^{m}$ so that $\Theta=\theta_{\chi, \Phi^{\prime}}^{m}$. It is sufficient to show that

$$
\int_{\mathfrak{a}_{p}} \mathcal{M}_{\chi}^{\kappa, m}\left(\left(w, x_{w}\right) t^{-1}\right) \Phi_{p}^{\prime} d_{\mathfrak{a}_{p}} w=|\mathrm{N}(1-t)|_{p}^{-1 / 2} \chi_{p}\left(\frac{1-t^{-1}}{\kappa}\right) \Phi_{p}^{\prime} .
$$

To simplify the notation, we omit the superscript $m$ and the subscript $p$, and write $\mathcal{M}_{\chi}$ for $\mathcal{M}_{\chi}^{\kappa, m}$. Let $V(\mathfrak{a})=\left\{\Phi \in V \mid \rho\left(w, x_{w}\right) \Phi=\Phi(w \in \mathfrak{a})\right\}=\left\{\Phi \in V \mid \mathcal{P}_{\mathfrak{a}} \Phi=\Phi\right\}$, where

$$
\mathcal{P}_{\mathfrak{a}} \Phi=\int_{\mathfrak{a}} \rho\left(w, x_{w}\right) \Phi d_{\mathfrak{a}} w
$$




\section{ON THE Fourier-JACOBI EXPANSION OF THE UNITARY KUDLA LIFT}

Observe that $V(\mathfrak{a})$ is one-dimensional since $\mu(m, \mathfrak{a})=0$ (cf. [MS00, Proposition 5.10]), and that both $\Phi_{p}^{\prime}$ and the integral of (4.1) belong to $V(\mathfrak{a})$. This implies

$$
\int_{\mathfrak{a}} \mathcal{M}_{\chi}\left(\left(w, x_{w}\right) t^{-1}\right) \Phi_{p}^{\prime} d_{\mathfrak{a}} w=\gamma(t) \Phi_{p}^{\prime}
$$

with $\gamma(t)=\operatorname{Tr}\left(\left.\mathcal{P}_{\mathfrak{a}} \mathcal{M}_{\chi}\left(t^{-1}\right)\right|_{V(\mathfrak{a})}\right)$. To calculate $\gamma(t)$, recall that $\Phi(z)=\left(\rho(z, 0) \Phi, \Phi_{0}\right)(\Phi \in V, z \in K)$, where $\Phi_{0}(z)=\operatorname{char}_{\mathcal{L}}(z) \psi_{m}\left(\frac{1}{4}\left\langle z, z^{\sigma}\right\rangle_{\kappa}\right) \in V$. It follows that $\mathcal{M}_{\chi}\left(t^{-1}\right) \Phi(z)$ is equal to

$$
\left(\rho(z, 0) \mathcal{M}_{\chi}\left(t^{-1}\right) \Phi, \Phi_{0}\right)=\left(\Phi, \mathcal{M}_{\chi}(t) \rho(-z, 0) \Phi_{0}\right)=c_{\chi}(t) \int_{K} \eta_{t}\left(z, z^{\prime}\right) \Phi\left(z^{\prime}\right) d^{m} z^{\prime}
$$

where

$$
c_{\chi}(t)=\overline{\lambda_{K}\left(\psi_{m}\right)^{-1} \chi\left(\frac{1-t}{\kappa}\right)|\mathrm{N}(1-t)|^{-1 / 2}}=\chi\left(\frac{1-t^{-1}}{\kappa}\right)|\mathrm{N}(1-t)|^{-1 / 2}
$$

and

$$
\begin{aligned}
\eta_{t}\left(z, z^{\prime}\right) & =\int_{K} \psi_{m}\left(-\frac{1}{2} \operatorname{Tr}\left(\frac{\kappa}{1-t}\right) \mathrm{N}\left(w^{\prime}\right)\right) \overline{\rho\left(w^{\prime}, 0\right) \rho(-z, 0) \Phi_{0}\left(z^{\prime}\right)} d^{m} w^{\prime} \\
& =\int_{K} \psi_{m}\left(-\frac{1}{2} \operatorname{Tr}\left(\frac{\kappa}{1-t}\right) \mathrm{N}\left(w^{\prime}\right)+\frac{1}{2}\left\langle w^{\prime}, z\right\rangle_{\kappa}-\frac{1}{2}\left\langle z^{\prime}, w^{\prime}-z\right\rangle_{\kappa}\right) \overline{\Phi_{0}\left(z^{\prime}-z+w^{\prime}\right)} d^{m} w^{\prime} .
\end{aligned}
$$

This implies that

with

$$
\mathcal{P}_{\mathfrak{a}} \mathcal{M}_{\chi}\left(t^{-1}\right) \Phi(z)=c_{\chi}(t) \int_{K} \eta_{t, \mathfrak{a}}\left(z, z^{\prime}\right) \Phi\left(z^{\prime}\right) d^{m} z^{\prime}
$$

$$
\eta_{t, \mathfrak{a}}\left(z, z^{\prime}\right)=\int_{\mathfrak{a}} \psi_{m}\left(\frac{1}{2}\langle z, w\rangle_{\kappa}+x_{w}\right) \eta_{t}\left(z+w, z^{\prime}\right) d_{\mathfrak{a}} w
$$

We thus have

$$
\begin{aligned}
\gamma(t)= & c_{\chi}(t) \int_{K} \eta_{t, \mathfrak{a}}(z, z) d^{m} z \\
= & c_{\chi}(t) \int_{\mathfrak{a}} \int_{K} \int_{K} \overline{\Phi_{0}\left(w^{\prime}-w\right)} \\
& \times \psi_{m}\left(\left\langle z, w-w^{\prime}\right\rangle_{\kappa}+x_{w}-\frac{1}{2} \operatorname{Tr}\left(\frac{\kappa}{1-t}\right) \mathrm{N}\left(w^{\prime}\right)+\frac{1}{2}\left\langle w^{\prime}, w\right\rangle_{\kappa}\right) d^{m} w^{\prime} d^{m} z d_{\mathfrak{a}} w \\
= & c_{\chi}(t) \overline{\Phi_{0}(0)} \int_{\mathfrak{a}} \psi_{m}\left(\frac{1-\kappa-(1+\kappa) t}{2(1-t)} \mathrm{N}(w)\right) d_{\mathfrak{a}} w \\
= & c_{\chi}(t) \int_{\mathfrak{a}} \psi_{m}\left(\frac{1-\kappa-(1+\kappa) t}{2(1-t)} \mathrm{N}(w)\right) d_{\mathfrak{a}} w .
\end{aligned}
$$

Since

$$
\frac{1-\kappa-(1+\kappa) t}{2(1-t)} \in \mathbb{Z}_{p} \quad \text { for } t \notin \mathcal{O}_{K, p}^{1},
$$

the last integral is equal to 1 and we have proved $\gamma(t)=c_{\chi}(t)$.

\section{Fourier-Jacobi component}

\subsection{Fourier-Jacobi expansion}

In this section, keeping the notation of $\S 4$, we recall several basic facts about Fourier-Jacobi expansion of automorphic forms on $G=U(S)$ after [Shi79] (see also [MS02]). 


\section{A. Murase And T. Sugano}

Let $F$ be a smooth function on $G_{\mathbb{Q}} \backslash G_{\mathbf{A}} / \mathcal{K}_{f}$. Then $F$ admits the Fourier-Jacobi expansion

$$
F(g)=\sum_{m \in \mathbb{Q}} F^{m}(g)
$$

where

$$
F^{m}(g)=\int_{\mathbb{Q} \backslash \mathbb{Q}_{\mathbf{A}}} F((0, x) g) \psi_{m}(-x) d x .
$$

For $\alpha \in K_{\mathbf{A}}^{\times}$, we define a function $F_{\alpha}^{m}$ on $R_{\mathbb{Q}} \backslash R_{\mathbf{A}}$ by

$$
F_{\alpha}^{m}(r)=F^{m}\left(r \mathbf{d}_{G}(\alpha)\right) \quad\left(r \in R_{\mathbf{A}}\right) .
$$

Note that $F_{\alpha}^{m} \in \mathbf{T}^{m}(\mathfrak{a})$ with $\mathfrak{a}=\operatorname{id}(\alpha)$ (for the definition of $\operatorname{id}(\alpha)$, see $\S 2.2$ ).

Proposition 5.1. Let $F$ be as above. Then $F$ is in $\mathfrak{S}_{l}\left(\mathcal{K}_{f}\right)$ if and only if the following four conditions are satisfied:

(5.1) $F\left(g k_{\infty}\right)=J\left(k_{\infty}, Z_{0}\right)^{-l} F(g)\left(k_{\infty} \in \mathcal{K}_{\infty}\right)$;

(5.2) $F^{m}=0$ if $m \leqslant 0$;

(5.3) for any $m \in \mathbb{Q}, m>0$ and $\alpha=\alpha_{\infty} \alpha_{f} \in K_{\mathbf{A}}^{\times}$, we have $F_{\alpha}^{m}=\alpha_{\infty}^{l} \mathbf{e}_{m}\left[(\kappa / 2)\left(\alpha_{\infty} \overline{\alpha_{\infty}}-1\right)\right] F_{\alpha_{f}}^{m}$;

(5.4) for any $m \in \mathbb{Q}, m>0$ and $\alpha_{f} \in K_{\mathbf{A}, f}^{\times}$, we have $F_{\alpha_{f}}^{m} \in \mathbf{T}_{\mathrm{hol}}^{m}$.

Proof. The necessity is easily verified. Suppose that the conditions (5.1)-(5.4) are satisfied. For $g_{f} \in G_{\mathbf{A}, f}$, we put $F_{d m}\left(g_{\infty}\left\langle Z_{0}\right\rangle ; g_{f}\right)=J\left(g_{\infty}, Z_{0}\right)^{l} F\left(g_{f} g_{\infty}\right)\left(g_{\infty} \in G_{\infty}\right)$. It is sufficient to show that $Z \mapsto F_{d m}\left(Z ; g_{f}\right)$ is holomorphic on $\mathcal{D}$ for any $g_{f} \in G_{\mathbf{A}, f}$. We may (and do) suppose that $g_{f}=r_{f} \mathbf{d}_{G}\left(\alpha_{f}\right)\left(r_{f} \in R_{\mathbf{A}, f}, \alpha_{f} \in K_{\mathbf{A}, f}^{\times}\right)$. For $Z={ }^{\mathrm{t}}(z, w) \in \mathcal{D}$, put $g_{Z}=(w, x) \mathbf{d}_{G}(\sqrt{y})$ with $z=x+(\kappa / 2)(y+w \bar{w})(x, y \in \mathbb{R}, y>0)$. Then $g_{Z}\left\langle Z_{0}\right\rangle=Z$. By (5.2) and (5.3), we obtain

$$
\begin{aligned}
F_{d m}\left(Z ; g_{f}\right) & =J\left(g_{Z}, Z_{0}\right)^{l} F\left(g_{f} g_{Z}\right) \\
& =y^{-l / 2} \sum_{m \in \mathbb{Q}, m>0, m \mathrm{~N}(\mathfrak{a}) \in \mathbb{Z}} F_{\alpha_{f} \sqrt{y}}^{m}\left(r_{f}(w, 0)\right) \mathbf{e}_{m}[x] \\
& =\sum_{m \in \mathbb{Q}, m>0, m \mathrm{~N}(\mathfrak{a}) \in \mathbb{Z}} F_{\alpha_{f}}^{m}\left(r_{f}(w, 0)\right) \cdot \mathbf{e}_{m}\left[-\frac{\kappa}{2}(w \bar{w}+1)\right] \mathbf{e}_{m}[z] .
\end{aligned}
$$

The condition (5.4) implies that each term in the sum is holomorphic in $Z$ and we are done.

\subsection{Primitive and non-primitive components}

Recall that $\mathcal{E}$ is the set of the triplets $(m, \mathfrak{a}, \chi)$, where $m$ is a positive rational number, $\mathfrak{a}$ an ideal of $K$ with $m \mathrm{~N}(\mathfrak{a}) \in \mathbb{Z}$ and $\chi \in \mathcal{X}_{\text {prim }}^{+}(m, \mathfrak{a})$. We put $\mu_{p}(m, \mathfrak{a})=\operatorname{ord}_{p} m \mathrm{~N}(\mathfrak{a})$. For $F \in \mathfrak{A}_{l}\left(\mathcal{K}_{f}\right),(m, \mathfrak{a}, \chi) \in \mathcal{E}$ and an ideal $\mathfrak{b}$ of $K$, we put

$$
C_{F}\left(\mathfrak{b} ; \Theta_{\mathfrak{a}, \chi}^{m}\right)=\left\langle\left\langle F_{\mathfrak{b}}^{m}, \Theta_{\mathfrak{a}, \chi}^{m}\right\rangle_{R},\right.
$$

where $\Theta_{\mathfrak{a}, \chi}^{m} \in \mathbf{T}_{\text {hol,prim }}^{m}(\mathfrak{a}, \chi)-\{0\}$ and $F_{\mathfrak{b}}^{m}=F_{\beta_{f}}^{m}$ with $\beta_{f} \in K_{\mathbf{A}, f}^{\times}, \operatorname{id}\left(\beta_{f}\right)=\mathfrak{b}$. Note that $\Theta_{\mathfrak{a}, \chi}^{m}$ is unique up to constant multiples by Theorem 4.4 and that $F_{\mathfrak{b}}^{m}$ does not depend on the choice of $\beta_{f}$. In view of Theorem $4.5, F$ is determined by $\left\{C_{F}\left(\mathfrak{b} ; \Theta_{\mathfrak{a}, \chi}^{m}\right) \mid(m, \mathfrak{a}, \chi) \in \mathcal{E}, \mathfrak{b} \subset \mathfrak{a}\right\}$, which we call the Fourier-Jacobi components of $F$. If $\mathfrak{b}=\mathfrak{a}$ (respectively $\mathfrak{b} \varsubsetneqq \mathfrak{a}$ ), the component $C_{F}\left(\mathfrak{b} ; \Theta_{\mathfrak{a}, \chi}^{m}\right)$ is said to be primitive (respectively non-primitive).

Lemma 5.2. Let $F \in \mathfrak{A}_{l}\left(\mathcal{K}_{f} ; \Omega^{-1}\right)$ and write $\Theta$ for $\Theta_{\mathfrak{a}, \chi}^{m}$.

(i) Suppose that $p$ is inert in $K / \mathbb{Q}$. Then $C_{F}\left(p^{k} \mathfrak{a} ; \Theta\right)=0$ unless $k \geqslant 0$.

(ii) Suppose that $p$ ramifies in $K / \mathbb{Q}$. Then $C_{F}\left(\Pi^{k} \mathfrak{a} ; \Theta\right)=0$ unless $k \geqslant 0$. 


\section{ON THE Fourier-JACOBI EXPANSION OF THE UNITARY KUDLA LIFT}

(iii) Suppose that $p$ splits in $K / \mathbb{Q}$. If $\mu_{p}(m, \mathfrak{a})>0$, we have $C_{F}\left(\Pi_{1}^{k_{1}} \Pi_{2}^{k_{2}} \mathfrak{a} ; \Theta\right)=0$ unless $k_{1}, k_{2} \geqslant 0$. If $\mu_{p}(m, \mathfrak{a})=0$, we have $C_{F}\left(\Pi_{1}^{k_{1}} \Pi_{2}^{k_{2}} \mathfrak{a} ; \Theta\right)=0$ unless $k_{1}+k_{2} \geqslant 0$.

(iv) Suppose that $p$ splits in $K / \mathbb{Q}$ and $\mu_{p}(m, \mathfrak{a})=0$. Then we have

$$
C_{F}\left(\Pi_{1}^{k_{1}} \Pi_{2}^{k_{2}} \mathfrak{a} ; \Theta\right)= \begin{cases}\left(p^{1 / 2} \chi\left(\Pi_{2}\right) \Omega^{-1}\left(\Pi_{1} / \Pi_{2}\right)\right)^{k_{2}} \cdot C_{F}\left(\Pi_{1}^{k_{1}+k_{2}} \mathfrak{a} ; \Theta\right) & \text { if } k_{1} \geqslant 0 \text { and } k_{2} \leqslant 0, \\ \left(p^{1 / 2} \chi\left(\Pi_{1}\right) \Omega\left(\Pi_{1} / \Pi_{2}\right)\right)^{k_{1}} \cdot C_{F}\left(\Pi_{2}^{k_{1}+k_{2}} \mathfrak{a} ; \Theta\right) & \text { if } k_{1} \leqslant 0 \text { and } k_{2} \geqslant 0 .\end{cases}
$$

Proof. The assertions (i)-(iii) are easily verified. To prove assertion (iv), we may (and do) assume that $k_{1}+k_{2} \geqslant 0$. Suppose that $k_{1} \geqslant 0, k_{2} \leqslant 0$, and put $t=\Pi_{1}^{-k_{2}} \Pi_{2}^{k_{2}}$ and $\beta=\Pi_{1}^{k_{1}+k_{2}} \alpha$. By Proposition 4.8, we have

$$
\begin{aligned}
\left\langle\left\langle F_{\Pi_{1}^{k_{1}} \Pi_{2}^{k_{2}} \mathfrak{a}}, \Theta\right\rangle\right\rangle_{R} \\
=\int_{R_{\mathbb{Q}} \backslash R_{\mathbf{A}}} F^{m}\left(r \mathbf{d}_{G}(\beta, t) t^{-1} 1_{3}\right) \overline{\Theta(r)} d r=\Omega(t) \int_{R_{\mathbb{Q}} \backslash R_{\mathbf{A}}} F^{m}\left(r \mathbf{d}_{G}(\beta)\right) \overline{\Theta\left(r t^{-1}\right)} d r \\
=\Omega(t) \int_{R_{\mathbb{Q}} \backslash R_{\mathbf{A}}} d r \int_{\mathbb{Z}_{p}} F^{m}\left(r\left(\left(0, \alpha_{2} y\right), 0\right) \mathbf{d}_{G}(\beta)\right) d y \int_{\mathbb{Z}_{p}} \overline{\Theta\left(r\left(\left(0, \alpha_{2} x\right), 0\right) t^{-1}\right)} d x \\
=\Omega(t) \int_{R_{\mathbb{Q}} \backslash R_{\mathbf{A}}} F^{m}\left(r \mathbf{d}_{G}(\beta)\right) d r \int_{\mathfrak{a}_{p}} \overline{\Theta\left(r\left(w, x_{w}\right) t^{-1}\right)} d x \\
=\Omega(t)|\mathrm{N}(1-t)|^{-1 / 2} \chi^{-1}\left(\frac{1-t^{-1}}{\kappa}\right) \int_{R_{\mathbb{Q}} \backslash R_{\mathbf{A}}} F^{m}\left(r \mathbf{d}_{G}(\beta)\right) \overline{\Theta(r)} d r \\
=\left(p^{1 / 2} \chi\left(\Pi_{2}\right) \Omega^{-1}\left(\Pi_{1} / \Pi_{2}\right)\right)^{k_{2}}\left\langle\left\langle F_{\Pi_{1}^{k_{1}+k_{2}}}^{m}, \Theta\right\rangle\right\rangle_{R} .
\end{aligned}
$$

This proves assertion (iv) in the case where $k_{1} \geqslant 0$ and $k_{2} \leqslant 0$. The proof in the remaining case is similar and omitted.

\subsection{Shintani's criterion}

In this subsection, we recall a criterion due to Shintani $\left[\right.$ Shi79, §3] for $F \in \mathfrak{S}_{l}\left(\mathcal{K}_{f} ; \Omega^{-1}\right)$ being a Hecke eigenform in terms of its Fourier-Jacobi components. (For the definitions of Hecke operators $\Phi_{i, p}$, see $\S 3.2$.)

Proposition 5.3. Suppose that $p$ is inert in $K / \mathbb{Q}$.

(i) We have $F * \Phi_{1, p}=\Lambda_{p} F$ with $\Lambda_{p} \in \mathbb{C}$ if and only if the following recursion relations hold for any $(m, \mathfrak{a}, \chi) \in \mathcal{E}$ and $k \geqslant 0$ :

$$
p^{4} c(k+1)+\left\{\delta\left(k+\mu_{p}(m, \mathfrak{a})>0\right) p-1-\Lambda_{p}\right\} c(k)+c(k-1)=0,
$$

where we put $c(k)=C_{F}\left(p^{k} \mathfrak{a} ; \Theta_{\mathfrak{a}, \chi}^{m}\right)$.

(ii) Suppose that part (i) holds. Then we have

$$
\sum_{k=0}^{\infty} c(k) X^{k}=\frac{1+\delta\left(\mu_{p}(m, \mathfrak{a})=0\right) p^{-3} X}{Q_{p}\left(p^{-2} X\right)} \cdot c(0)
$$

where $Q_{p}(X)=1-p^{-2}\left(\Lambda_{p}-p+1\right) X+X^{2}$.

Proposition 5.4. Suppose that $p$ ramifies in $K / \mathbb{Q}$.

(i) We have $F * \Phi_{1, p}=\Lambda_{p} F$ with $\Lambda_{p} \in \mathbb{C}$ if and only if the following recursion relations hold for any $(m, \mathfrak{a}, \chi) \in \mathcal{E}$ and $k \geqslant 0$ :

$$
p^{2} c(k+1)+\left\{\delta(k>0 \text { or } \chi \text { is exceptional }) \cdot p-1-\Lambda_{p}\right\} c(k)+c(k-1)=0,
$$

where we put $c(k)=C_{F}\left(\Pi^{k} \mathfrak{a} ; \Theta_{\mathfrak{a}, \chi}^{m}\right)$. 


\section{A. Murase and T. Sugano}

(ii) Suppose that part (i) holds. Then we have

$$
\sum_{k=0}^{\infty} c(k) X^{k}=\frac{1+\delta(\chi \text { is ordinary }) p^{-1} X}{Q_{p}\left(p^{-1} X\right)} \cdot c(0),
$$

where $Q_{p}(X)=1-p^{-1}\left(\Lambda_{p}-p+1\right) X+X^{2}$.

Proposition 5.5. Suppose that $p$ splits in $K / \mathbb{Q}$.

(i) We have $F * \Phi_{i, p}=\Lambda_{i, p} F$ with $\Lambda_{i, p} \in \mathbb{C}(i=1,2)$ if and only if the following recursion relations hold for any $(m, \mathfrak{a}, \chi) \in \mathcal{E}$ and $k_{1}, k_{2} \geqslant 0$ :

$$
\begin{aligned}
& \Lambda_{1, p} \cdot c\left(k_{1}, k_{2}\right)=p^{2} c\left(k_{1}, k_{2}+1\right)+p \Omega^{-1}\left(\Pi_{1} / \Pi_{2}\right) \cdot c\left(k_{1}+1, k_{2}-1\right)+c\left(k_{1}-1, k_{2}\right), \\
& \Lambda_{2, p} \cdot c\left(k_{1}, k_{2}\right)=p^{2} c\left(k_{1}+1, k_{2}\right)+p \Omega\left(\Pi_{1} / \Pi_{2}\right) \cdot c\left(k_{1}-1, k_{2}+1\right)+c\left(k_{1}, k_{2}-1\right),
\end{aligned}
$$

where we put $c\left(k_{1}, k_{2}\right)=C_{F}\left(\Pi_{1}^{k_{1}} \Pi_{2}^{k_{2}} \mathfrak{a} ; \Theta_{\mathfrak{a}, \chi}^{m}\right)$.

(ii) Suppose that part (i) holds. Then we have

$$
\sum_{k_{1}, k_{2}=0}^{\infty} c\left(k_{1}, k_{2}\right) X_{1}^{k_{1}} X_{2}^{k_{2}}=\frac{R\left(X_{1}, X_{2}\right)}{Q_{1}\left(p^{-1} X_{1}\right) Q_{2}\left(p^{-1} X_{2}\right)} \cdot c(0,0),
$$

where

$$
\begin{aligned}
Q_{1}(X) & =1-p^{-1} \Lambda_{2, p} X+p^{-1} \Omega\left(\Pi_{1} / \Pi_{2}\right) \Lambda_{1, p} X^{2}-\Omega\left(\Pi_{1} / \Pi_{2}\right) X^{3}, \\
Q_{2}(X) & =1-p^{-1} \Lambda_{1, p} X+p^{-1} \Omega^{-1}\left(\Pi_{1} / \Pi_{2}\right) \Lambda_{2, p} X^{2}-\Omega^{-1}\left(\Pi_{1} / \Pi_{2}\right) X^{3}, \\
R\left(X_{1}, X_{2}\right) & =\left(1-p^{-2} X_{1} X_{2}\right) \prod_{i=1,2}\left(1-\delta\left(\mu_{p}(m, \mathfrak{a})=0\right) \chi^{-1}\left(\Pi_{i}\right) p^{-3 / 2} X_{i}\right) .
\end{aligned}
$$

Theorem $5.6\left[\right.$ Shi79, §3]. Suppose that $F \in \mathfrak{A}_{l}\left(\mathcal{K}_{f} ; \Omega^{-1}\right)$ is a Hecke eigenform. Then $F=0$ if and only if $C_{F}\left(\mathfrak{a} ; \Theta_{\mathfrak{a}, \chi}^{m}\right)=0$ for any $(m, \mathfrak{a}, \chi) \in \mathcal{E}$.

Proof. This follows from Lemma 5.2, Propositions 5.3-5.5 and Theorem 4.5.

\section{Metaplectic representation}

6.1 In this section, we let $F$ be either the $p$-adic number field $\mathbb{Q}_{p}$ or the real number field $\mathbb{R}$. When $F=\mathbb{Q}_{p}$, let $K$ be a quadratic extension of $F$ or $F \oplus F$. When $F=\mathbb{R}$, let $K=\mathbb{C}$. Denote by $\sigma$ the nontrivial automorphism of $K / F$. For $z \in K$, put $\operatorname{Tr}(z)=z+z^{\sigma}$ and $\mathrm{N}(z)=z z^{\sigma}$. Denote by $|\cdot|_{F}$ the normalized valuation of $F$. Let $\psi$ be the additive character of $F$ given by

$$
\psi(x)= \begin{cases}\mathbf{e}[-(\text { the fractional part of } x)] & \text { if } F=\mathbb{Q}_{p}, \\ \mathbf{e}[x] & \text { if } F=\mathbb{R} .\end{cases}
$$

Recall that $d x$ (respectively $d z$ ) is the Haar measure on $F$ (respectively $K$ ) self-dual with respect to the pairing $\left(x, x^{\prime}\right) \mapsto \psi\left(x x^{\prime}\right)$ (respectively $\left(z, z^{\prime}\right) \mapsto \psi\left(\operatorname{Tr}\left(z^{\sigma} z^{\prime}\right)\right)$ ). Denote by $\lambda_{K}(\psi)$ the Weil constant attached to $(K / F, \psi)$ (cf. $\S 4.3)$. We have $\lambda_{K}(\psi)=\sqrt{-1}$ if $F=\mathbb{R}$.

Let $F=\mathbb{Q}_{p}$. Denote by $\mathcal{O}_{F}$ the integer ring of $F$ and let

$$
\mathcal{O}_{K}= \begin{cases}\text { the integer ring of } K & \text { if } K \text { is a field, } \\ \mathcal{O}_{F} \oplus \mathcal{O}_{F} & \text { if } K=F \oplus F\end{cases}
$$

Let $\mathcal{X}$ be the set of characters of $K^{\times}$whose restriction to $F^{\times}$coincides with $\omega=\omega_{K / F}$, the character of $F^{\times}$corresponding to $K / F$ by local class field theory. 


\subsection{Dual reductive pair}

Let $\mathbf{H}=U(\mathbf{T})$ be the unitary group of a non-degenerate anti-hermitian matrix

$$
\mathbf{T}=\left[\begin{array}{ccc} 
& \kappa^{-1} T \\
-\kappa^{-1} T &
\end{array}\right]
$$

Define a homomorphism of $G \times H$ to $\mathbf{H}$ by

$$
(g, h) \mapsto g \otimes h:=\left(g_{i j} 1_{2}\right)_{1 \leqslant i, j \leqslant 3} \cdot\left[\begin{array}{lll}
h & & \\
& h & \\
& & h
\end{array}\right] \quad\left(g=\left(g_{i j}\right) \in G, h \in H\right) .
$$

Note that the kernel of the homomorphism is $\left\{\left(t 1_{3}, t^{-1} 1_{2}\right) \mid t \in K^{1}\right\}$.

\subsection{Heisenberg group and representation}

Let $\langle\cdot, \cdot\rangle_{T}$ and $\langle\cdot, \cdot\rangle_{\mathbf{T}}$ be the $F$-alternating forms on $W=K^{2}$ and $\mathbf{W}=K^{6}$ attached to $T$ and $\mathbf{T}$, respectively (see $\S 2.2$ ). Let $\mathcal{N}_{T}$ and $\mathcal{N}_{\mathbf{T}}$ be the Heisenberg groups attached to $\left(W,\langle\cdot, \cdot\rangle_{T}\right)$ and $\left(\mathbf{W},\langle\cdot, \cdot\rangle_{\mathbf{T}}\right)$, respectively:

$$
\begin{gathered}
\mathcal{N}_{T}=W \ltimes F,(w, x)\left(w^{\prime}, x^{\prime}\right)=\left(w+w^{\prime}, x+x^{\prime}+\frac{1}{2}\left\langle w, w^{\prime}\right\rangle_{T}\right), \\
\mathcal{N}_{\mathbf{T}}=\mathbf{W} \ltimes F,(\boldsymbol{w}, x)\left(\boldsymbol{w}^{\prime}, x^{\prime}\right)=\left(\boldsymbol{w}+\boldsymbol{w}^{\prime}, x+x^{\prime}+\frac{1}{2}\left\langle\boldsymbol{w}, \boldsymbol{w}^{\prime}\right\rangle_{\mathbf{T}}\right) .
\end{gathered}
$$

Define a smooth representation $\rho_{T}$ (respectively $\rho_{\mathbf{T}}$ ) of $\mathcal{N}_{T}$ (respectively $\mathcal{N}_{\mathbf{T}}$ ) on $V=\mathcal{S}(K)$ (respectively $\mathbf{V}=\mathcal{S}\left(K^{2}\right) \otimes \mathcal{S}(K)$ ) as follows:

$$
\rho_{T}\left(\left(\begin{array}{l}
w_{1} \\
w_{2}
\end{array}\right), x\right) \varphi(z)=\psi\left(-\operatorname{Tr}\left(w_{1}^{\sigma} z\right)-\frac{1}{2} \operatorname{Tr}\left(w_{1}^{\sigma} w_{2}\right)+x\right) \varphi\left(z+w_{2}\right),
$$

for $w_{1}, w_{2}, z \in K, x \in F, \varphi \in \mathcal{S}(K)$;

$$
\begin{aligned}
& \rho_{\mathbf{T}}\left(\left[\begin{array}{l}
\boldsymbol{w}_{1} \\
\boldsymbol{w}_{2} \\
\boldsymbol{w}_{3}
\end{array}\right], x\right)(\Phi \otimes \varphi)(Z, z) \\
& \quad=\psi\left(-\frac{1}{2} \operatorname{Tr}\left(\kappa^{-1} T\left(\boldsymbol{w}_{1}, \boldsymbol{w}_{3}\right)\right)-\operatorname{Tr}\left(\kappa^{-1} T\left(\boldsymbol{w}_{1}, Z\right)\right)+x\right) \times \Phi\left(Z+\boldsymbol{w}_{3}\right)\left(\rho_{T}\left(\boldsymbol{w}_{2}, 0\right) \varphi\right)(z)
\end{aligned}
$$

for $\boldsymbol{w}_{i} \in K^{2}(i=1,2,3), x \in F, \Phi \in \mathcal{S}\left(K^{2}\right), \varphi \in \mathcal{S}(K), Z \in K^{2}, z \in K$. Then $\rho_{T}$ and $\rho_{\mathbf{T}}$ are irreducible, and any smooth irreducible representation of $\mathcal{N}_{T}$ (respectively $\mathcal{N}_{\mathbf{T}}$ ) with central character $(0, x) \mapsto \psi(x)$ is equivalent to $\rho_{T}$ (respectively $\left.\rho_{\mathbf{T}}\right)$.

\subsection{Metaplectic representations}

Let $\chi_{0} \in \mathcal{X}$. We can define a splitting $\mathcal{M}_{\chi_{0}}^{T}$ (respectively $\mathcal{M}_{\chi_{0}}^{\mathbf{T}}$ ) of metaplectic representation of $H=U(T)$ (respectively $\mathbf{H}=U(\mathbf{T})$ ) attached to $\left(\rho_{T}, \chi_{0}\right)$ (respectively $\left(\rho_{\mathbf{T}}, \chi_{0}\right)$ ) as in [Mur01]. The following two lemmas are proved by a straightforward calculation and we omit their proofs. We note that $\mathcal{M}_{\chi_{0}}^{\mathbf{T}}$ is a mixed model of the metaplectic representation of $\mathbf{H}$.

Lemma 6.1. Let $\varphi \in \mathcal{S}(K)$ and $z \in K$. Then

$$
\begin{gathered}
\mathcal{M}_{\chi_{0}}^{T}\left(\mathbf{d}_{H}(a)\right) \varphi(z)=\chi_{0}^{-1}(a)|\mathrm{N}(a)|_{F}^{1 / 2} \varphi(a z) \quad\left(a \in K^{\times}\right), \\
\mathcal{M}_{\chi_{0}}^{T}\left(\mathbf{n}_{H}(b)\right) \varphi(z)=\psi\left(b z z^{\sigma}\right) \varphi(z) \quad(b \in F) \\
\mathcal{M}_{\chi_{0}}^{T}\left(w_{0}\right) \varphi(z)=\lambda_{K}(\psi) \widehat{\varphi}(z)
\end{gathered}
$$




\section{A. Murase and T. Sugano}

where

$$
\widehat{\varphi}(z)=\int_{K} \psi\left(\operatorname{Tr}\left(w^{\sigma} z\right)\right) \varphi(w) d w .
$$

Lemma 6.2. Let $\Phi \in \mathcal{S}\left(K^{2}\right), \varphi \in \mathcal{S}(K), Z \in K^{2}$ and $z \in K$. Then

$$
\begin{gathered}
\mathcal{M}_{\chi_{0}}^{\mathbf{T}}\left(1_{3} \otimes h\right)(\Phi \otimes \varphi)(Z, z)=\chi_{0}(\operatorname{det} h) \Phi\left(h^{-1} Z\right)\left(\mathcal{M}_{\chi_{0}}^{T}(h) \varphi\right)(z) \quad(h \in H), \\
\mathcal{M}_{\chi_{0}}^{\mathbf{T}}\left((w, x) \otimes 1_{2}\right)(\Phi \otimes \varphi)(Z, z)=\psi\left(x \cdot \kappa^{-1} T[Z]\right) \Phi(Z)\left(\rho_{T}(-w Z, 0) \varphi\right)(z) \quad(w \in K, x \in F), \\
\mathcal{M}_{\chi_{0}}^{\mathbf{T}}\left(\mathbf{d}_{G}(a, t) \otimes 1_{2}\right)(\Phi \otimes \varphi)(Z, z)=\chi_{0}^{-2}(a) \chi_{0}(t)|\mathrm{N}(a)|_{F} \Phi(a Z) \varphi\left(t^{-1} z\right) \quad\left(a \in K^{\times}, t \in K^{1}\right), \\
\mathcal{M}_{\chi_{0}}^{\mathbf{T}}\left(\left[\begin{array}{ccc} 
& & 1 \\
& &
\end{array}\right] \otimes 1_{2}\right)(\Phi \otimes \varphi)(Z, z)=\widehat{\Phi}(Z) \varphi(z),
\end{gathered}
$$

where

$$
\widehat{\Phi}(Z)=\int_{K^{2}} \psi\left(\operatorname{Tr}\left(\kappa^{-1} T\left(Z, Z^{\prime}\right)\right)\right) \Phi\left(Z^{\prime}\right) d Z^{\prime} .
$$

Here $d Z^{\prime}$ is the Haar measure on $K^{2}$ self-dual with respect to the pairing $\left(Z, Z^{\prime}\right) \mapsto \psi\left(\operatorname{Tr}\left(\kappa^{-1}\right.\right.$ $\left.\left.T\left(Z, Z^{\prime}\right)\right)\right)$.

Lemma 6.3. Suppose that $F=\mathbb{Q}_{p}$ and let $\varphi_{0, p}$ be the characteristic function of $\mathcal{O}_{K}$. Then we have $\mathcal{M}_{\chi_{0}}^{T}(u) \varphi_{0, p}=\tilde{\chi}_{0}(u) \varphi_{0, p}$ for $u \in \mathcal{U}_{p}^{\prime}$ (for the definition of $\tilde{\chi}_{0}$, see $\S 3.3$ ).

Proof. Put $V_{0}=\left\{\varphi \in V \mid \rho_{T}(l, \alpha(l)) \varphi=\varphi\right.$ for any $\left.l \in L^{\prime}\right\}$, where $\alpha(w)=\frac{1}{2} \operatorname{Tr}\left(w_{1}^{\sigma} w_{2}\right)$ for $w=$ ${ }^{\mathrm{t}}\left(w_{1}, w_{2}\right) \in W$ and $L^{\prime}={ }^{\mathrm{t}}\left(\sqrt{D}^{-1} \mathcal{O}_{K}, \mathcal{O}_{K}\right)$ is a lattice of $W$. It is easily verified that $V_{0}=\mathbb{C} \varphi_{0, p}$ and $\mathcal{M}_{\chi_{0}}^{T}(u) V_{0} \subset V_{0}$ for $u \in \mathcal{U}_{p}^{\prime}$. Thus, $\mathcal{M}_{\chi_{0}}^{T}(u) \varphi_{0, p}=c_{\chi_{0}}(u) \varphi_{0, p}\left(u \in \mathcal{U}_{p}^{\prime}\right)$ with $c_{\chi_{0}}(u) \in \mathbb{C}^{\times}$. A direct calculation shows that $c_{\chi_{0}}\left(\mathbf{n}_{H}(b)\right)=1\left(b \in \mathcal{O}_{F}\right), c_{\chi_{0}}\left(\overline{\mathbf{n}}_{H}(b)\right)=1\left(b \in D \mathcal{O}_{F}\right)$ and $c_{\chi_{0}}\left(\mathbf{d}_{H}(a)\right)=$ $\chi_{0}^{-1}(a)\left(a \in \mathcal{O}_{K}^{\times}\right)$. This immediately implies that $c_{\chi_{0}}(u)=\tilde{\chi}_{0}(u)\left(u \in \mathcal{U}_{p}^{\prime}\right)$.

Remark. This lemma gives another proof of Lemma 3.2.

\subsection{Intertwining operator}

We now describe a relation between our mixed model and the Schrödinger model used in [Kud81]. Define a linear isomorphism $i: K^{3} \times K^{3} \rightarrow K^{6}$ by $i\left(z^{+}, z^{-}\right)={ }^{\mathrm{t}}\left(z_{1}^{+}, z_{1}^{-}, z_{2}^{+}, z_{2}^{-}, z_{3}^{+}, z_{3}^{-}\right)\left(z^{ \pm}=\right.$ $\left.{ }^{\mathrm{t}}\left(z_{1}^{ \pm}, z_{2}^{ \pm}, z_{3}^{ \pm}\right) \in K^{3}\right)$. We then have $\left\langle i\left(z^{+}, z^{-}\right), i\left(w^{+}, w^{-}\right)\right\rangle_{\mathbf{T}}=\operatorname{Tr}\left(S\left(z^{+}, w^{-}\right)-S\left(z^{-}, w^{+}\right)\right)$. Let $\widetilde{\rho}_{\mathbf{T}}$ be the smooth irreducible representation of $\mathcal{N}_{\mathbf{T}}$ on $\mathcal{S}\left(K^{3}\right)$ corresponding to a complete polarization $\mathbf{W}=i\left(K^{3},\{0\}\right) \oplus i\left(\{0\}, K^{3}\right)$ :

$$
\widetilde{\rho}_{\mathbf{T}}\left(i\left(w^{+}, w^{-}\right), x\right) f(z)=\psi\left(x-\operatorname{Tr} S\left(w^{+}, z\right)-\frac{1}{2} \operatorname{Tr} S\left(w^{+}, w^{-}\right)\right) f\left(z+w^{-}\right)
$$

for $w^{ \pm}, z \in K^{3}, x \in F, f \in \mathcal{S}\left(K^{3}\right)$. Let $\widetilde{\mathcal{M}}_{\chi_{0}}^{\mathbf{T}}: \mathbf{H} \rightarrow G L\left(\mathcal{S}\left(K^{3}\right)\right)$ be the metaplectic representation attached to $\left(\widetilde{\rho}_{\mathbf{T}}, \chi_{0}\right)$. Then we have

$$
\begin{gathered}
\widetilde{\mathcal{M}}_{\chi_{0}}^{\mathbf{T}}\left(g \otimes 1_{2}\right) f(z)=\chi_{0}(\operatorname{det} g) f\left(g^{-1} z\right) \quad(g \in G), \\
\widetilde{\mathcal{M}}_{\chi_{0}}^{\mathbf{T}}\left(1_{3} \otimes \mathbf{d}_{H}(a)\right) f(z)=\chi_{0}^{-3}(a)|\mathrm{N}(a)|_{F}^{3 / 2} f(a z) \quad\left(a \in K^{\times}\right), \\
\widetilde{\mathcal{M}}_{\chi_{0}}^{\mathbf{T}}\left(1_{3} \otimes \mathbf{n}_{H}(b)\right) f(z)=\psi(b S[z]) f(z) \quad(b \in F), \\
\widetilde{\mathcal{M}}_{\chi_{0}}^{\mathbf{T}}\left(1_{3} \otimes w_{0}\right) f(z)=\lambda_{K}(\psi) \widehat{f}(z),
\end{gathered}
$$

where

$$
\widehat{f}(z)=\int_{K^{3}} \psi(\operatorname{Tr} S(z, w)) f(w) d_{S} w
$$




\section{ON THE Fourier-JACOBI EXPANSION OF THE UNITARY KUDLA LIFT}

$\left(d_{S} w\right.$ is the Haar measure self-dual with respect to the pairing $\left.\left(w, w^{\prime}\right) \mapsto \psi\left(\operatorname{Tr} S\left(w, w^{\prime}\right)\right)\right)$. Note that $\widetilde{\mathcal{M}}_{\chi_{0}}^{\mathbf{T}}$ is used in [Kud81] to construct the theta kernel. Define $I: \mathcal{S}\left(K^{2}\right) \otimes \mathcal{S}(K) \rightarrow \mathcal{S}\left(K^{3}\right)$ by

$$
I(\Phi \otimes \varphi)(z)=\int_{K} \psi\left(\operatorname{Tr}\left(\kappa^{-1} z_{1}^{\sigma} u\right)\right) \Phi\left(\left(\begin{array}{c}
u \\
z_{3}
\end{array}\right)\right) d u \cdot \varphi\left(z_{2}\right) \quad\left(z={ }^{\mathrm{t}}\left(z_{1}, z_{2}, z_{3}\right) \in K^{3}\right) .
$$

We easily see that $I \circ \rho_{\mathbf{T}}(\boldsymbol{w}, x)=\widetilde{\rho}_{\mathbf{T}}(\boldsymbol{w}, x) \circ I \quad(\boldsymbol{w} \in \mathbf{W}, x \in F)$, which directly implies

$$
I \circ \mathcal{M}_{\chi_{0}}^{\mathbf{T}}(\boldsymbol{h})=\widetilde{\mathcal{M}}_{\chi_{0}}^{\mathbf{T}}(\boldsymbol{h}) \circ I \quad(\boldsymbol{h} \in \mathbf{H}) .
$$

\subsection{Test functions}

When $F=\mathbb{Q}_{p}$, we set $\Phi_{0, p}=\operatorname{char}_{\mathcal{O}_{K}^{2}}$ and $\varphi_{0, p}=\operatorname{char}_{\mathcal{O}_{K}}$. When $F=\mathbb{R}$, we set

$$
\begin{gathered}
\Phi_{0, \infty}\left(\begin{array}{l}
z_{1} \\
z_{2}
\end{array}\right)=\left(z_{1}-\sqrt{-1} z_{2}\right)^{l} \mathbf{e}\left[\frac{\sqrt{-1}}{2}\left(z_{1} \overline{z_{1}}+z_{2} \overline{z_{2}}\right)\right] \quad\left(z_{1}, z_{2} \in \mathbb{C}\right), \\
\varphi_{0, \infty}(z)=\mathbf{e}[\sqrt{-1} z \bar{z}] \quad(z \in \mathbb{C}) .
\end{gathered}
$$

Set $\Phi_{0}=\bigotimes_{v} \Phi_{0, v} \in \mathcal{S}\left(K_{\mathbf{A}}^{2}\right)$ and $\varphi_{0}=\bigotimes_{v} \varphi_{0, v} \in \mathcal{S}\left(K_{\mathbf{A}}\right)$. A straightforward calculation shows the following.

Lemma 6.4. We have $I\left(\Phi_{0, p} \otimes \varphi_{0, p}\right)=|D|_{p}^{1 / 2} \operatorname{char}_{\mathcal{O}_{K, p}^{3}}$ for $p<\infty$, and

$$
I\left(\Phi_{0, \infty} \otimes \varphi_{0, \infty}\right)\left({ }^{\mathrm{t}}\left(z_{1}, z_{2}, z_{3}\right)\right)=2 \sqrt{-1}^{l}\left(\frac{2}{\sqrt{D}} z_{1}+z_{3}\right)^{l} \mathbf{e}\left[\sqrt{-1}\left\{-\frac{2}{D} z_{1} \overline{z_{1}}+z_{2} \overline{z_{2}}+\frac{1}{2} z_{3} \overline{z_{3}}\right\}\right] .
$$

Using Lemma 6.4, we obtain the following result.

Proposition 6.5. We have the following.

(i) Let $F=\mathbb{Q}_{p}$. Then we have

$$
\mathcal{M}_{\chi_{0}}^{\mathbf{T}}(k \otimes u)\left(\Phi_{0, p} \otimes \varphi_{0, p}\right)=\chi_{0}(\operatorname{det} k) \chi_{0}^{2}(\operatorname{det} u) \widetilde{\chi}_{0}(u)^{-1} \Phi_{0, p} \otimes \varphi_{0, p} \quad\left(k \in \mathcal{K}_{p}, u \in \mathcal{U}_{p}^{\prime}\right) .
$$

(ii) Let $F=\mathbb{R}$ and put $\lambda=w_{\infty}\left(\chi_{0}\right)$. Then we have

$$
\begin{aligned}
& \mathcal{M}_{\chi_{0}}^{\mathbf{T}}(k \otimes u)\left(\Phi_{0, \infty} \otimes \varphi_{0, \infty}\right) \\
& \quad=(\operatorname{det} k)^{\lambda} J\left(k, Z_{0}\right)^{-l}(\operatorname{det} u)^{(3 \lambda+1) / 2-l} j\left(u, z_{0}\right)^{l-1} \Phi_{0, \infty} \otimes \varphi_{0, \infty} \quad\left(k \in \mathcal{K}_{\infty}, u \in \mathcal{U}_{\infty}\right) .
\end{aligned}
$$

\section{Main results}

\subsection{Theta kernel}

We now go back to the global situation. Recall that we have fixed a $\chi_{0} \in \mathcal{X}$ with $w_{\infty}\left(\chi_{0}\right)=-1$. Let $\mathcal{M}_{\chi_{0}}^{\mathbf{T}}$ be the metaplectic representation of $\mathbf{H}_{\mathbf{A}}$ on $\mathcal{S}\left(K_{\mathbf{A}}^{2}\right) \otimes \mathcal{S}\left(K_{\mathbf{A}}\right)$ given by

$$
\mathcal{M}_{\chi_{0}}^{\mathbf{T}}(\boldsymbol{h})=\bigotimes_{v} \mathcal{M}_{\chi_{0, v}}^{\mathbf{T}}\left(\boldsymbol{h}_{v}\right) \quad\left(\boldsymbol{h}=\left(\boldsymbol{h}_{v}\right)_{v} \in \mathbf{H}_{\mathbf{A}}\right)
$$

where the local components $\mathcal{M}_{\chi_{0, v}}^{\mathbf{T}}$ are defined in $\S 6.4$. Define a theta kernel on $G_{\mathbf{A}} \times H_{\mathbf{A}}$ by

$$
\theta(g, h)=\chi_{0}^{-1}(\operatorname{det} g) \chi_{0}^{-2}(\operatorname{det} h) \sum_{X \in K^{2}, \xi \in K} \mathcal{M}_{\chi_{0}}^{\mathbf{T}}(g \otimes h)\left(\Phi_{0} \otimes \varphi_{0}\right)(X, \xi)
$$

for $g \in G_{\mathbf{A}}, h \in H_{\mathbf{A}}($ cf. $\S 6.6)$.

LEMMA 7.1. We have

$$
\theta\left(\gamma g k_{f} k_{\infty}, \gamma^{\prime} h u_{f} u_{\infty}\right)=\tilde{\chi}_{0}\left(u_{f}\right)^{-1} J\left(k_{\infty}, Z_{0}\right)^{-l} j^{\prime}\left(u_{\infty}, z_{0}\right)^{l-1} \theta(g, h)
$$

for $\gamma \in G_{\mathbb{Q}}, g \in G_{\mathbf{A}}, k_{f} \in \mathcal{K}_{f}, k_{\infty} \in \mathcal{K}_{\infty}, \gamma^{\prime} \in H_{\mathbb{Q}}, h \in H_{\mathbf{A}}, u_{f} \in \mathcal{U}_{f}^{\prime}, u_{\infty} \in \mathcal{U}_{\infty}$. 


\section{A. Murase And T. Sugano}

Proof. The left $G_{\mathbb{Q}} \times H_{\mathbb{Q}}$-invariance is proved by a Poisson summation formula in the usual way. The remaining part directly follows from Proposition 6.5.

\subsection{The unitary Kudla lift}

For $f \in S_{l-1}\left(\mathcal{U}_{f}^{\prime}, \widetilde{\chi}_{0}\right)$ (cf. $\left.\S 3.3\right)$, we put

$$
\mathcal{L} f(g)=\int_{H_{\mathbb{Q} \backslash H_{\mathbf{A}}}} f(h) \theta(g, h) d h \quad\left(g \in G_{\mathbf{A}}\right) .
$$

The integral is absolutely convergent since $f$ is rapidly decreasing on $H_{\mathbb{Q}} \backslash H_{\mathbf{A}}$. The theta lift (7.1) was first studied by Kudla [Kud79, Kud81] and we call $\mathcal{L} f$ the unitary Kudla lift of $f$. Lemma 7.1 implies

$$
\mathcal{L} f\left(\gamma g k_{f} k_{\infty}\right)=J\left(k_{\infty}, Z_{0}\right)^{-l} \mathcal{L} f(g)
$$

for $\gamma \in G_{\mathbb{Q}}, g \in G_{\mathbf{A}}, k_{f} \in \mathcal{K}_{f}, k_{\infty} \in \mathcal{K}_{\infty}$. In [Kud79], it is proved that

$$
\mathcal{L} f \in \mathfrak{S}_{l}\left(\mathcal{K}_{f}\right)
$$

for $f \in S_{l-1}\left(\mathcal{U}_{f}^{\prime}, \widetilde{\chi}_{0}\right)$ by showing that the lifts of Poincaré series in $S_{l-1}\left(\mathcal{U}_{f}^{\prime}, \widetilde{\chi}_{0}\right)$ are holomorphic. Later we give another proof of $(7.3)$ by studying the Fourier-Jacobi expansion of $\mathcal{L} f$ (see Theorem 7.4). Note that

$$
\mathcal{L} f\left(t 1_{3} \cdot g\right)=\Omega^{-1}(t) \mathcal{L} f(g) \quad\left(t \in K_{\mathbf{A}}^{1}, g \in G_{\mathbf{A}}\right)
$$

for $f \in S_{l-1}\left(\mathcal{U}_{p}^{\prime}, \widetilde{\chi}_{0} ; \chi_{0} \Omega\right)$, since $\theta\left(t 1_{3} \cdot g, h\right)=\chi_{0}(t) \theta\left(g, t 1_{2} \cdot h\right)$ for $t \in K_{\mathbf{A}}^{1}$.

Remark. Although the realization of the metaplectic representation here is different from that in [Kud81], the theta kernels coincide up to a constant multiple in view of $\S \S 6.5$ and 6.6.

7.3 For $m \in \mathbb{Q}^{\times}$, put

$$
\eta_{m}=\left(\begin{array}{c}
m \theta^{\sigma} \\
1
\end{array}\right)
$$

(for the definition of $\theta$, see $\S 2.2$ ). Let $H_{\eta_{m}}=\left\{h \in H \mid h \eta_{m}=\eta_{m}\right\}$ be the stabilizer subgroup of $\eta_{m}$ in $H$. For $t=z^{\sigma} / z \in K^{1}\left(z=x+y \theta \in K^{\times}, x, y \in \mathbb{Q}\right)$, we put

$$
\iota_{m}(t)=\frac{1}{z}\left(\begin{array}{cc}
x & m N(\theta) y \\
-y / m & x+\operatorname{Tr}(\theta) y
\end{array}\right) .
$$

The following fact is easily verified.

LEMma 7.2. We have the following.

(i) For $t \in K^{1}$, we have $\iota_{m}(t) \in H_{\eta_{m}, \mathbb{Q}}$ and $\operatorname{det} \iota_{m}(t)=t$.

(ii) The mapping $\iota_{m}$ defines an isomorphism of $K^{1}$ onto $H_{\eta_{m}, \mathbb{Q}}$.

(iii) We have $\left\{X \in K^{2} \mid \kappa^{-1} T[X]=m\right\}=\left\{\gamma^{-1} \eta_{m} \mid \gamma \in H_{\eta_{m}, \mathbb{Q}} \backslash H_{\mathbb{Q}}\right\}$.

\subsection{Spherical functions}

Let $f \in S_{l-1}\left(\mathcal{U}_{f}^{\prime}, \widetilde{\chi}_{0}\right)$. For $m \in \mathbb{Q}^{\times}$and $\chi \in \mathcal{X}$, set

$$
W_{\chi, f}^{m}(h)=\int_{K^{1} \backslash K_{\mathbf{A}}^{1}}\left(\chi / \chi_{0}\right)^{1}\left(t^{-1}\right) f\left(\iota_{m}(t) h\right) d^{\times} t \quad\left(h \in H_{\mathbf{A}}\right)
$$

(for the definition of $\left(\chi / \chi_{0}\right)^{1}$, see $\left.\S 2.5\right)$. 


\section{ON THE Fourier-JACOBI EXPANSION OF THE UNITARY KUDLA LIFT}

\subsection{Holomorphy and cuspidality of the unitary Kudla lift}

The following fact is proved in $\S \S 9.2$ and 9.4 .

Proposition 7.3. Let $f \in S_{l-1}\left(\mathcal{U}_{f}^{\prime}, \widetilde{\chi}_{0}\right)$ and $F=\mathcal{L} f$.

(i) If $m \leqslant 0$, we have $F^{m}=0$.

(ii) For any $m \in \mathbb{Q}, m>0$ and any $\alpha=\alpha_{\infty} \alpha_{f} \in K_{\mathbf{A}}^{\times}$, we have $F_{\alpha}^{m}=\alpha_{\infty}^{l} \mathbf{e}_{m}\left[2^{-1} \kappa\left(\alpha_{\infty} \overline{\alpha_{\infty}}-1\right)\right] F_{\alpha_{f}}^{m}$ and $F_{\alpha_{f}}^{m} \in \mathbf{T}_{\text {hol }}^{m}$.

(For the definitions of $F^{m}$ and $F_{\alpha}^{m}$, see $\S$ 5.1.)

Theorem $7.4\left[\operatorname{Kud79}\right.$, Theorem 5.3]. For $f \in S_{l-1}\left(\mathcal{U}_{f}^{\prime}, \tilde{\chi}_{0} ; \chi_{0} \Omega\right)$, we have $\mathcal{L} f \in \mathfrak{S}_{l}\left(\mathcal{K}_{f} ; \Omega^{-1}\right)$.

Proof. This follows from Propositions 5.1 and 7.3 and (7.4).

\subsection{Main results: primitive components}

Let $(m, \mathfrak{a}, \chi) \in \mathcal{E}(\mathrm{cf} . \S 4.8)$. Take and fix an element $h_{m \theta, \infty}$ of $H_{\infty}$ with $h_{m \theta, \infty}\left\langle z_{0}\right\rangle=m \theta$. Set

$$
\begin{aligned}
& c_{\infty}=4 \pi \sqrt{-1}^{l}(m|\kappa|)^{l-2} \mathbf{e}\left[\frac{m \kappa}{2}\right] j^{\prime}\left(h_{m \theta, \infty}, z_{0}\right)^{l-1}, \\
& c_{p}=p^{\mu_{p}(m, \mathfrak{a})-\delta_{p} / 2} \times \begin{cases}1 & \text { if } \delta_{p}=0, \mu_{p}(m, \mathfrak{a})=0, \\
1-\omega_{p}\left(\pi_{p}\right) p^{-1} & \text { if } \delta_{p}=0, \mu_{p}(m, \mathfrak{a})>0, \\
\frac{2 p}{1+p} & \text { if } \delta_{p}>0 .\end{cases}
\end{aligned}
$$

Note that $c_{p}=1$ except for a finite number of $p$. Denote by $E(\chi)$ the set of finite primes $p$ such that $p \mid D$ and $\chi_{p}$ is exceptional (cf. $\left.\S 4.10\right)$. For $\epsilon=\left(\epsilon_{p}\right)_{p \in E(\chi)} \in\{0,1\}^{E(\chi)}$, we put

$$
w_{0}^{\epsilon}=\prod_{p \in E(\chi)} w_{0, p}^{\epsilon_{p}} \quad\left(w_{0, p}=\left(\begin{array}{rr}
0 & 1 \\
-1 & 0
\end{array}\right) \in H_{p}\right), \quad B(\chi, \epsilon)=\prod_{p \in E(\chi)}\left(p^{-1 / 2} \chi_{p}^{-1}(m \theta)\right)^{\epsilon_{p}} .
$$

We set

$$
J(m, \mathfrak{a}, \chi, f)=\sum_{\epsilon \in\{0,1\}^{E(\chi)}} B(\chi, \epsilon) W_{\chi, f}^{m}\left(\mathbf{d}_{H}\left(\alpha_{f}^{-1}\right) w_{0}^{\epsilon} h_{m \theta, \infty}\right),
$$

where we choose $\alpha_{f} \in K_{\mathbf{A}, f}^{\times}$so that $\operatorname{id}\left(\alpha_{f}\right)=\mathfrak{a}$.

Remark. Suppose that $K$ has class number 1. Let $f_{d m}$ be as in the remark in $\S 3.4$. Then we have, for any $h_{f} \in H_{\mathbf{A}, f}$,

$$
W_{\chi, f}^{m}\left(h_{f} h_{m \theta, \infty}\right)=\sum_{j=1}^{N} a_{j} f_{d m}\left(\tau_{j}\right),
$$

where $a_{j} \in \mathbb{C}$ (depending only on $m, \chi, h_{f}$ ) and $\tau_{j} \in K \cap \mathfrak{H}$ (depending only on $m, h_{f}$ ). It follows that $J(m, \mathfrak{a}, \chi, f)$ is a linear combination of CM-values of $f_{d m}$. A similar assertion holds (with suitable modifications) when $h_{K}>1$.

Theorem 7.5 (Main Theorem I). Let $(m, \mathfrak{a}, \chi) \in \mathcal{E}, \Theta \in \mathbf{T}_{\text {hol,prim }}^{m}(\mathfrak{a}, \chi)$ and $f \in S_{l-1}\left(\mathcal{U}_{f}^{\prime}, \widetilde{\chi}_{0}\right)$. Then the primitive component (cf. $\S 5.2)$ of the unitary Kudla lift $\mathcal{L} f$ is given by

$$
C_{\mathcal{L} f}(\mathfrak{a} ; \Theta)=c_{\infty} \prod_{p<\infty} c_{p} \cdot \chi_{0}^{-1}\left(\alpha_{f}\right)\left|\mathrm{N}\left(\alpha_{f}\right)\right|^{3 / 2} \cdot \overline{I(\Theta)} \cdot J(m, \mathfrak{a}, \chi, f) .
$$




\section{A. Murase and T. Sugano}

\subsection{Main results: non-primitive components}

Theorem 7.6 (Main Theorem II). Let $f \in S_{l-1}\left(\mathcal{U}_{p}^{\prime}, \widetilde{\chi}_{0} ; \chi_{0} \Omega\right)$ be a Hecke eigenform with eigenvalues $\left\{\lambda_{p}\right\}$. Let $p$ be a finite prime, $(m, \mathfrak{a}, \chi) \in \mathcal{E}$ and $\Theta \in \mathbf{T}_{\text {hol,prim }}^{m}(\mathfrak{a}, \chi)$. We write $\mu_{p}$ for $\mu_{p}(m, \mathfrak{a})$.

(i) Suppose that $p$ is inert in $K / \mathbb{Q}$. Set $c(k)=C_{\mathcal{L} f}\left(p^{k} \mathfrak{a} ; \Theta\right)$. Then

$$
p^{4} c(k+1)+\left\{\delta\left(k+\mu_{p}>0\right) p-p^{2}-p \lambda_{p}\left(\phi_{1, p}\right)\right\} c(k)+c(k-1)=0
$$

holds for $k \geqslant 0$.

(ii) Suppose that $p$ ramifies in $K / \mathbb{Q}$. Set $c(k)=C_{\mathcal{L} f}\left(\Pi^{k} \mathfrak{a} ; \Theta\right)$. Then

$$
p^{2} c(k+1)-\left\{p^{1 / 2} \lambda_{p}\left(\phi_{p}^{+}+\phi_{p}^{-}\right)+p \cdot \delta(k=0 \text { and } \chi \text { is ordinary })\right\} c(k)+c(k-1)=0
$$

holds for $k \geqslant 0$.

(iii) Suppose that $p$ splits in $K / \mathbb{Q}$. Set $c\left(k_{1}, k_{2}\right)=C_{\mathcal{L} f}\left(\Pi_{1}^{k_{1}} \Pi_{2}^{k_{2}} \mathfrak{a} ; \Theta\right)$. Then

$p^{2} c\left(k_{1}, k_{2}+1\right)-\left(p^{1 / 2} \lambda_{p}\left(\phi_{2, p}\right)+p\right) c\left(k_{1}, k_{2}\right)+p \Omega^{-1}\left(\Pi_{1} / \Pi_{2}\right) c\left(k_{1}+1, k_{2}-1\right)+c\left(k_{1}-1, k_{2}\right)=0$

and

$$
p^{2} c\left(k_{1}+1, k_{2}\right)-\left(p^{1 / 2} \lambda_{p}\left(\phi_{1, p}\right)+p\right) c\left(k_{1}, k_{2}\right)+p \Omega\left(\Pi_{1} / \Pi_{2}\right) c\left(k_{1}-1, k_{2}+1\right)+c\left(k_{1}, k_{2}-1\right)=0
$$

hold for $k_{1}, k_{2} \geqslant 0$.

Combining Theorem 7.6, Propositions 5.3-5.5 and Theorem 5.6, we obtain the following results.

Corollary 7.7. Let $f \in S_{l-1}\left(\mathcal{U}_{p}^{\prime}, \widetilde{\chi}_{0} ; \chi_{0} \Omega\right)$ be a Hecke eigenform with eigenvalues $\left\{\lambda_{p}\right\}$.

(i) The unitary Kudla lift $\mathcal{L} f$ is a Hecke eigenform with eigenvalues $\left\{\Lambda_{p}\right\}$, where $\Lambda_{p}$ is given as follows.

(a) If $p$ is inert in $K / \mathbb{Q}$, we have $\Lambda_{p}\left(\Phi_{1, p}\right)=p \lambda_{p}\left(\phi_{1, p}\right)+p^{2}-1$.

(b) If $p$ ramifies in $K / \mathbb{Q}$, we have $\Lambda_{p}\left(\Phi_{1, p}\right)=p^{1 / 2} \lambda_{p}\left(\phi_{p}^{+}+\phi_{p}^{-}\right)+p-1$.

(c) If $p$ splits in $K / \mathbb{Q}$, we have $\Lambda_{p}\left(\Phi_{1, p}\right)=p^{1 / 2} \lambda_{p}\left(\phi_{2, p}\right)+p$ and $\Lambda_{p}\left(\Phi_{2, p}\right)=p^{1 / 2} \lambda_{p}\left(\phi_{1, p}\right)+p$.

(ii) For a Hecke character $\xi$ of $K$, we have

$$
L(\mathcal{L} f, \xi ; s)=L(\xi ; s) L(f, \xi ; s) .
$$

(For the definition of L-functions, see $\S \S 3.2$ and 3.4.)

Remark. Corollary 7.7 was proved by Kudla [Kud81, Corollary 1] by using Eichler commutation relations.

Theorem 7.8 (Main Theorem III). Let $f \in S_{l-1}\left(\mathcal{U}_{p}^{\prime}, \widetilde{\chi}_{0} ; \chi_{0} \Omega\right)$ be a Hecke eigenform. The unitary Kudla lift $\mathcal{L} f$ does not vanish if and only if there exists $(m, \mathfrak{a}, \chi) \in \mathcal{E}$ such that

$$
L\left(\chi ; \frac{1}{2}\right) \cdot J(m, \mathfrak{a}, \chi, f) \neq 0 .
$$

Proof. This follows from Theorems 5.6, 7.5 and 4.7 .

\section{Integral expression of the Fourier-Jacobi components}

8.1 Let $f \in S_{l-1}\left(\mathcal{U}_{f}^{\prime}, \tilde{\chi}_{0}\right)$. The object of this section is to give an integral expression of the FourierJacobi components of $\mathcal{L} f$. By Lemma 6.2, we have

$$
\begin{aligned}
\mathcal{L} f\left((w, x) t \mathbf{d}_{G}(\alpha)\right)= & \chi_{0}^{-1}(t)|\mathrm{N}(\alpha)| \mathbf{A} \int_{H_{\mathbb{Q}} \backslash H_{\mathbf{A}}} \sum_{X \in K^{2}, \xi \in K} \chi_{0}^{-1}(\operatorname{det} h) \psi\left(\kappa^{-1} T[X] x\right) \\
& \times \Phi_{0}\left(\alpha h^{-1} X\right)\left(\mathcal{M}_{\chi_{0}}^{T}(h) \rho_{T}\left(-w h^{-1} X, 0\right) \mathcal{M}_{\chi_{0}}^{T}\left(t 1_{2}\right) \varphi_{0}\right)(\xi) f(h) d h
\end{aligned}
$$

for $(w, x) \in N_{\mathbf{A}}, t \in K_{\mathbf{A}}^{1}$ and $\alpha \in K_{\mathbf{A}}^{\times}$(for the definition of $\mathcal{M}_{\chi_{0}}^{T}$ and $\rho_{T}$, see $\S \S 6.4$ and 6.3). 


\subsection{The constant term}

Proposition 8.1. We have $(\mathcal{L} f)^{0}=0$.

Proof. Let $\eta^{\prime}={ }^{\mathrm{t}}(1,0) \in K^{2}$ and $N^{\prime}=N^{H}$. Since $\left\{X \in K^{2} \mid T[X]=0\right\}=\left\{\gamma^{-1} \eta^{\prime} \mid \gamma \in N_{\mathbb{Q}}^{\prime} \backslash H_{\mathbb{Q}}\right\}$, we have

$$
\begin{aligned}
(\mathcal{L} f)^{0}\left((w, 0) t \mathbf{d}_{G}(\alpha)\right)= & \chi_{0}^{-1}(t)|\mathrm{N}(\alpha)|_{\mathbf{A}} \int_{H_{\mathbb{Q}} \backslash H_{\mathbf{A}}} \sum_{\gamma \in N_{\mathbb{Q}}^{\prime} \backslash H_{\mathbb{Q}}} \chi_{0}^{-1}(\operatorname{det} \gamma h) \Phi_{0}\left(\alpha(\gamma h)^{-1} \eta^{\prime}\right) \\
& \times \sum_{\xi \in K}\left(\mathcal{M}_{\chi_{0}}^{T}(h) \rho_{T}\left(-w(\gamma h)^{-1} \eta^{\prime}, 0\right) \mathcal{M}_{\chi_{0}}^{T}\left(t 1_{2}\right) \varphi_{0}\right)(\xi) f(h) d h .
\end{aligned}
$$

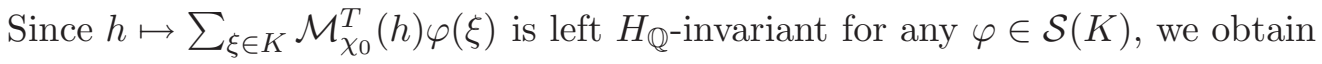

$$
\begin{aligned}
& (\mathcal{L} f)^{0}\left((w, 0) t \mathbf{d}_{G}(\alpha)\right) \\
& \quad=\chi_{0}^{-1}(t)|\mathrm{N}(\alpha)|_{\mathbf{A}} \int_{N_{\mathbb{Q}}^{\prime} \backslash H_{\mathbf{A}}} \chi_{0}^{-1}(\operatorname{det} h) \Phi_{0}\left(\alpha h^{-1} \eta^{\prime}\right) \sum_{\xi \in K}\left(\rho_{T}\left(-w \eta^{\prime}, 0\right) \mathcal{M}_{\chi_{0}}^{T}(t h) \varphi_{0}\right)(\xi) f(h) d h \\
& \quad=\chi_{0}^{-1}(t)|\mathrm{N}(\alpha)|_{\mathbf{A}} \int_{N_{\mathbb{Q}}^{\prime} \backslash H_{\mathbf{A}}} \chi_{0}^{-1}(\operatorname{det} h) \Phi_{0}\left(\alpha h^{-1} \eta^{\prime}\right) \sum_{\xi \in K} \psi\left(\operatorname{Tr}\left(w^{\sigma} \xi\right)\right)\left(\mathcal{M}_{\chi_{0}}^{T}(t h) \varphi_{0}\right)(\xi) f(h) d h \\
& \quad=\chi_{0}^{-1}(t)|\mathrm{N}(\alpha)|_{\mathbf{A}} \int_{N_{\mathbf{A}}^{\prime} \backslash H_{\mathbf{A}}} \chi_{0}^{-1}(\operatorname{det} h) \Phi_{0}\left(\alpha h^{-1} \eta^{\prime}\right) \sum_{\xi \in K} \psi\left(\operatorname{Tr}\left(w^{\sigma} \xi\right)\right)\left(\mathcal{M}_{\chi_{0}}^{T}(t h) \varphi_{0}\right)(\xi) c_{f}(-N(\xi) ; h) d h .
\end{aligned}
$$

Here we set

$$
c_{f}(b ; h)=\int_{\mathbb{Q} \backslash \mathbb{Q}_{\mathbf{A}}} \psi(-b x) f\left(\mathbf{n}_{H}(x) h\right) d x \quad\left(h \in H_{\mathbf{A}}\right)
$$

for $b \in \mathbb{Q}$ and $h \in H_{\mathbf{A}}$. We have $c_{f}(-\mathrm{N}(\xi) ; h)=0$ for $\xi \in K$ by holomorphy and cuspidality of $f$. This implies that $(\mathcal{L} f)^{0}\left((w, 0) t \mathbf{d}_{G}(\alpha)\right)=0$ and we are done.

\subsection{Non-constant terms}

Let $m \in \mathbb{Q}^{\times}$and $\beta \in K_{\mathbf{A}}^{\times}$. Then $(\mathcal{L} f)_{\beta}^{m} \in \mathbf{T}^{m}(\mathfrak{b}) \cap \mathbf{T}^{m, 0}$ with $\mathfrak{b}=\operatorname{id}(\beta)$. Let $\chi \in \mathcal{X}$. For $\Theta \in \mathbf{T}_{\chi}^{m}$, we set

$$
\Lambda_{\beta, \Theta}^{m}(h)=|\mathrm{N}(\beta)|_{\mathbf{A}} \chi_{0}^{-1}(\operatorname{det} h) \Phi_{0}\left(\beta h^{-1} \eta_{m}\right) \int_{K_{\mathbf{A}}}\left(\mathcal{M}_{\chi_{0}}^{T}(h) \varphi_{0}\right)(-w) \overline{I_{\Theta}\left(w, x_{w}\right)} d w
$$

(for the definition of $I_{\Theta}$, see $\S 4.11$ ).

Lemma 8.2. For $\Theta \in \mathbf{T}_{\chi}^{m}$, we have

$$
\left\langle\left\langle(\mathcal{L} f)_{\beta}^{m}, \Theta\right\rangle\right\rangle_{R}=\int_{H_{\eta_{m}, \mathbb{Q} \backslash H_{\mathbf{A}}}} \Lambda_{\beta, \Theta}^{m}(h) f(h) d h .
$$

Proof. By Lemma 7.2 and an argument similar to that in $\S 8.2$, we have

$$
\begin{aligned}
(\mathcal{L} f)_{\beta}^{m}((w, 0) t)= & \chi_{0}^{-1}(t)|\mathrm{N}(\beta)|_{\mathbf{A}} \int_{H_{\eta_{m}, \mathbb{Q}} \backslash H_{\mathbf{A}}} \chi_{0}^{-1}(\operatorname{det} h) \Phi_{0}\left(\beta h^{-1} \eta_{m}\right) \\
& \times \sum_{\xi \in K} \psi\left(\operatorname{Tr}\left(m \theta \xi w^{\sigma}\right)-m x_{w}\right)\left(\mathcal{M}_{\chi_{0}}^{T}(t h) \varphi_{0}\right)(\xi-w) f(h) d h
\end{aligned}
$$

for $w \in K_{\mathbf{A}}$ and $t \in K_{\mathbf{A}}^{1}$. The left $R_{\mathbb{Q}^{-i n v a r i a n c e ~ o f ~} \Theta \text { implies that }}$

$$
\Theta((w, 0) t)=\psi\left(\frac{1}{2} \operatorname{Tr}\left(m \kappa \xi w^{\sigma}\right)\right) \Theta((w-\xi, 0) t)
$$




\section{A. Murase And T. Sugano}

for $\xi \in K$. We thus have

$$
\begin{aligned}
& \left\langle\left\langle(\mathcal{L} f)_{\beta}^{m}, \Theta\right\rangle\right\rangle_{R} \\
& =|\mathrm{N}(\beta)|_{\mathbf{A}} \int_{H_{\eta_{m}, \mathbb{Q}} \backslash H_{\mathbf{A}}} \int_{K \backslash K_{\mathbf{A}}} \int_{K^{1} \backslash K_{\mathbf{A}}^{1}} \chi_{0}^{-1}(t \operatorname{det} h) \Phi_{0}\left(\beta h^{-1} \eta_{m}\right) f(h) \\
& \times \sum_{\xi \in K} \psi\left(-\frac{m \operatorname{Tr}(\theta)}{2}(w-\xi)(w-\xi)^{\sigma}\right)\left(\mathcal{M}_{\chi_{0}}^{T}(t h) \varphi_{0}\right)(\xi-w) \overline{\Theta((w-\xi, 0) t)} d^{\times} t d w d h \\
& =|\mathrm{N}(\beta)|_{\mathbf{A}} \int_{H_{\eta_{m}, \mathbb{Q}} \backslash H_{\mathbf{A}}} \int_{K_{\mathbf{A}}} \int_{K^{1} \backslash K_{\mathbf{A}}^{1}} \chi_{0}^{-1}(t \operatorname{det} h) \Phi_{0}\left(\beta h^{-1} \eta_{m}\right) f(h) \\
& \times\left(\mathcal{M}_{\chi_{0}}^{T}(t h) \varphi_{0}\right)(-w) \overline{\Theta\left(\left(w, x_{w}\right) t\right)} d^{\times} t d w d h \\
& =|\mathrm{N}(\beta)|_{\mathbf{A}} \int_{H_{\eta_{m}, \mathbb{Q}} \backslash H_{\mathbf{A}}} \int_{K_{\mathbf{A}}} \int_{K^{1} \backslash K_{\mathbf{A}}^{1}} \chi_{0}^{-1}(\operatorname{det} h) \Phi_{0}\left(\beta h^{-1} \eta_{m}\right) f(h) \\
& \times\left(\mathcal{M}_{\chi_{0}}^{T}(h) \varphi_{0}\right)\left(-t^{-1} w\right) \overline{\Theta\left(t\left(t^{-1} w, x_{t^{-1} w}\right)\right)} d^{\times} t d w d h \\
& =\int_{H_{\eta_{m}, \mathbb{Q}} \backslash H_{\mathbf{A}}} \Lambda_{\beta, \Theta}^{m}(h) f(h) d h .
\end{aligned}
$$

Lemma 8.3 (Key lemma). Let $v$ be a prime of $\mathbb{Q}$. Let $\varphi \in \mathcal{S}\left(K_{v}\right)$ and $\Theta \in \mathbf{T}_{\chi}^{m}$. Then, for $t \in K_{v}^{1}$ and $r \in R_{\mathbf{A}}$, we have

$$
\int_{K_{v}}\left(\mathcal{M}_{\chi_{0}}^{T}\left(\iota_{m}(t)\right) \varphi\right)(-w) \overline{\Theta\left(r\left(w, x_{w}\right)\right)} d w=\left(\chi \chi_{0}\right)^{1}\left(t^{-1}\right) \int_{K_{v}} \varphi(-w) \overline{\Theta\left(r t\left(w, x_{w}\right)\right)} d w .
$$

Proof. If $t=1$, the assertion is trivial. Suppose that $t \neq 1$. Take $\Phi \in V^{m}$ such that $\Theta=\theta_{\chi, \Phi}^{m}$ (cf. $\S 4.4$ ). It is sufficient to show the equality

$$
\int_{K_{v}}\left(\mathcal{M}_{\chi_{0}}^{T}\left(\iota_{m}(t)\right) \varphi\right)(-w) \overline{\mathcal{M}_{\chi}^{\kappa, m}\left(r\left(w, x_{w}\right)\right) \Phi} d w=\left(\chi \chi_{0}\right)^{1}\left(t^{-1}\right) \int_{K_{v}} \varphi(-w) \overline{\mathcal{M}_{\chi}^{\kappa, m}\left(r t\left(w, x_{w}\right)\right) \Phi} d w .
$$

Take an element $x+y \theta$ of $K_{v}^{\times}$with $x, y \in \mathbb{Q}_{v}(y \neq 0)$ such that $t=(x+y \theta)^{\sigma} /(x+y \theta)$. Observe that

$$
T\left(\iota_{m}(t)-1_{2}\right)=\frac{y}{x+y \theta}\left(\begin{array}{cc}
-m^{-1} & \theta^{\sigma} \\
\theta & -m N(\theta)
\end{array}\right)=\frac{y}{x+y \theta}\left(A^{*}\right)^{-1}\left(\begin{array}{cc}
0 & 0 \\
0 & m \kappa^{2}
\end{array}\right) A^{-1}
$$

with

$$
A=\left(\begin{array}{cc}
m \theta^{\sigma} & m \theta \\
1 & 1
\end{array}\right)
$$

and that we can take $\left\{\zeta_{m}={ }^{\mathrm{t}}(m \theta, 1)\right\}$ as a basis of $K_{v}^{2} / \operatorname{Ker}\left(\iota_{m}(t)-1_{2}\right)$. Note that $\iota_{m}(t) \zeta_{m}=t \zeta_{m}$. In view of [Mur01, Theorem 1.8], we have

$$
\begin{aligned}
& \left(\mathcal{M}_{\chi_{0}}^{T}\left(\iota_{m}(t)\right) \varphi\right)(-w) \\
& \quad=\gamma(t) \int_{K_{v}} \psi\left(\frac{1}{2}\left\langle z \zeta_{m}, z \iota_{m}(t) \zeta_{m}\right\rangle_{T}\right) \rho_{T}\left(z\left(1_{2}-\iota_{m}(t)\right) \zeta_{m}, 0\right) \varphi(-w) d z \\
& \quad=\gamma(t) \int_{K_{v}} \psi_{m}\left(-\frac{1}{2} \operatorname{Tr}(\kappa t) z z^{\sigma}+\operatorname{Tr}\left(\theta^{\sigma}\left(1-t^{\sigma}\right) z^{\sigma} w\right)-x_{(1-t) z}\right) \varphi(-w+z(1-t)) d z
\end{aligned}
$$

where

$$
\begin{aligned}
\gamma(t) & =|\mathrm{N}(m \kappa(1-t))|_{v}^{1 / 2} \lambda_{K_{v}}\left(\psi_{v}\right)^{-1} \chi_{0}\left(\frac{y}{x+y \theta} m \kappa^{2}\right) \\
& =|\mathrm{N}(m \kappa(1-t))|_{v}^{1 / 2} \overline{\lambda_{K_{v}}\left(\psi_{m, v}\right)^{-1}} \chi_{0}\left(\frac{1-t}{\kappa}\right) .
\end{aligned}
$$


It follows that the left-hand side of $(*)$ is equal to

$$
\begin{aligned}
\gamma(t) \int_{K_{v}} \int_{K_{v}} \psi_{m}\left(-\frac{1}{2} \operatorname{Tr}(\kappa t) z z^{\sigma}+\operatorname{Tr}\left(\theta^{\sigma}\left(1-t^{\sigma}\right) z^{\sigma} w\right)-x_{(1-t) z}-x_{w}\right) \\
\quad \times \varphi(-w+z(1-t)) \overline{\mathcal{M}_{\chi}^{\kappa, m}(r(w, 0)) \Phi} d z d w \\
=\chi_{0}\left(\frac{1-t}{\kappa}\right)|\mathrm{N}(m \kappa(1-t))|_{v}^{1 / 2} \int_{K_{v}} \varphi(-w) \\
\quad \times \lambda_{K_{v}}\left(\psi_{m, v}\right) \overline{\int_{K_{v}} \psi_{m}\left(\frac{1}{2}\langle z, t z\rangle_{\kappa}\right) \mathcal{M}_{\chi}^{\kappa, m}\left(r((1-t) z, 0)\left(w, x_{w}\right)\right) \Phi d z} d w \\
=\left(\chi_{\chi_{0}}\right)\left(\frac{1-t}{\kappa}\right) \int_{K_{v}} \varphi(-w) \overline{\mathcal{M}_{\chi}^{\kappa, m}\left(r t\left(w, x_{w}\right)\right) \Phi} d w .
\end{aligned}
$$

Since $\left(\chi_{0}\right)((1-t) / \kappa)=\left(\chi \chi_{0}\right)^{1}\left(t^{-1}\right)\left(t \in K_{\mathbf{A}}^{1}\right)$, we are done.

Proposition 8.4. Let $m \in \mathbb{Q}^{\times}, \beta \in K_{\mathbf{A}}^{\times}, \chi \in \mathcal{X}$ and $\Theta \in \mathbf{T}_{\chi}^{m}$.

(i) We have $\Lambda_{\beta, \Theta}^{m}\left(\iota_{m}(t) h\right)=\left(\chi / \chi_{0}\right)^{1}\left(t^{-1}\right) \Lambda_{\beta, \Theta}^{m}(h)\left(t \in K_{\mathbf{A}}^{1}, h \in H_{\mathbf{A}}\right)$.

(ii) For $f \in S_{l-1}\left(\mathcal{U}_{f}^{\prime}, \tilde{\chi}_{0}\right)$, we have

$$
\left\langle\left\langle(\mathcal{L} f)_{\beta}^{m}, \Theta\right\rangle\right\rangle_{R}=\int_{H_{\eta_{m}, \mathbf{A}} \backslash H_{\mathbf{A}}} \Lambda_{\beta, \Theta}^{m}(h) W_{\chi, f}^{m}(h) d h,
$$

where $W_{\chi, f}^{m}$ is defined by (7.7) and the Haar measure $d x$ on $H_{\eta_{m}, \mathbf{A}}$ is normalized by

$$
\int_{H_{\eta_{m}, \mathbf{A}}} \varphi(x) d x=\int_{K_{\mathbf{A}}^{1}} \varphi\left(\iota_{m}(t)\right) d^{\times} t \quad\left(\varphi \in L^{1}\left(H_{\eta_{m}, \mathbf{A}}\right)\right) .
$$

Proof. The proposition is a direct consequence of Lemmas 8.2 and 8.3.

\section{Holomorphy and cuspidality}

\subsection{Real spherical functions}

For $m \in \mathbb{Q}^{\times}$and $k \in \mathbb{Z}$, let $\mathcal{W}_{\infty}^{m}(k)$ be the space of smooth functions $W$ on $H_{\infty}$ satisfying:

(i) $W\left(\iota_{m}(t) h u\right)=t^{k} j^{\prime}\left(u, z_{0}\right)^{1-l} W(h)\left(t \in K_{\infty}^{1}, h \in H_{\infty}, u \in \mathcal{U}_{\infty}\right)$;

(ii) $\widetilde{W}\left(h\left\langle z_{0}\right\rangle\right):=j^{\prime}\left(h, z_{0}\right)^{l-1} W(h)$ is holomorphic on $\mathfrak{H}$.

\subsection{Inverse Cayley transform}

Let $\mathbf{D}=\{w \in \mathbb{C}|| w \mid<1\}$ be the unit disc in $\mathbb{C}$. We define the inverse Cayley transform $C_{m}: \mathbf{D} \rightarrow \mathfrak{H}$ by

$$
C_{m}(w)= \begin{cases}\frac{m\left(\theta-\theta^{\sigma} w\right)}{1-w} & \text { if } m>0, \\ \frac{m\left(\theta^{\sigma}-\theta w\right)}{1-w} & \text { if } m<0\end{cases}
$$

$(w \in \mathbf{D})$. The following fact is easily verified. 


\section{A. Murase And T. Sugano}

Lemma 9.1. Let $t \in K_{\infty}^{1}, w \in \mathbf{D}$ and $W \in \mathcal{W}_{\infty}^{m}(k)$. Then we have

$$
\begin{aligned}
\iota_{m}(t)\left\langle C_{m}(w)\right\rangle & = \begin{cases}C_{m}\left(t^{-1} w\right) & \text { if } m>0, \\
C_{m}(t w) & \text { if } m<0,\end{cases} \\
j^{\prime}\left(\iota_{m}(t), C_{m}(w)\right) & = \begin{cases}\frac{1-t^{-1} w}{1-w} & \text { if } m>0, \\
\frac{t^{-1}-w}{1-w} & \text { if } m<0,\end{cases} \\
\widetilde{W}\left(\iota_{m}(t)\left\langle C_{m}(w)\right\rangle\right) & = \begin{cases}t^{k}\left(\frac{1-t^{-1} w}{1-w}\right)^{l-1} \widetilde{W}\left(C_{m}(w)\right) & \text { if } m>0, \\
t^{k-l+1}\left(\frac{1-t w}{1-w}\right)^{l-1} \widetilde{W}\left(C_{m}(w)\right) & \text { if } m<0 .\end{cases}
\end{aligned}
$$

Lemma 9.2. Let $W \in \mathcal{W}_{\infty}^{m}(k)$ and $z \in \mathfrak{H}$.

(i) If $m>0$, we have

$$
\widetilde{W}(z)= \begin{cases}c(z-m \theta)^{-k}\left(z-m \theta^{\sigma}\right)^{k-l+1} & \text { if } k \leqslant 0 \\ 0 & \text { if } k>0\end{cases}
$$

with $c \in \mathbb{C}$.

(ii) If $m<0$, we have

$$
\widetilde{W}(z)= \begin{cases}c(z-m \theta)^{-k}\left(z-m \theta^{\sigma}\right)^{k-l+1} & \text { if } k \geqslant l-1 \\ 0 & \text { if } k<l-1\end{cases}
$$

with $c \in \mathbb{C}$.

Proof. First assume that $m>0$. Put $W^{*}(w)=(1-w)^{1-l} \widetilde{W}\left(C_{m}(w)\right)$ for $w \in \mathbf{D}$. Then $W^{*}$ is holomorphic on $\mathbf{D}$ and satisfies $W^{*}\left(t^{-1} w\right)=t^{k} W^{*}(w)$ for $t \in K_{\infty}^{1}$ by Lemma 9.1. It follows that $W^{*}(w)=c^{\prime} \delta(k \leqslant 0) w^{-k}$ with $c^{\prime} \in \mathbb{C}$. Since $w=(z-m \theta) /\left(z-m \theta^{\sigma}\right)$ and $1-w=m\left(\theta-\theta^{\sigma}\right) /\left(z-m \theta^{\sigma}\right)$, we have proved assertion (i). The assertion (ii) can be proved similarly.

\subsection{Global spherical functions}

In this subsection, we let $f \in S_{l-1}\left(\mathcal{U}_{f}^{\prime}, \widetilde{\chi}_{0}\right)$ and $m \in \mathbb{Q}^{\times}$. Recall that, for $\chi \in \mathcal{X}, W_{\chi, f}^{m}$ is defined by (7.7).

Proposition 9.3. We have the following.

(i) If $m>0$ and $w_{\infty}(\chi)<-1$, we have $W_{\chi, f}^{m}=0$.

(ii) If $m<0$ and $w_{\infty}(\chi)>1-2 l$, we have $W_{\chi, f}^{m}=0$.

Proof. Observe that $h_{\infty} \mapsto W_{\chi, f}^{m}\left(h_{f} h_{\infty}\right)$ belongs to $\mathcal{W}_{\infty}^{m}\left(-\left(w_{\infty}(\chi)+1\right) / 2\right)$ for any $h_{f} \in H_{\mathbf{A}, f}$. Then the proposition directly follows from Lemma 9.2.

Proposition 9.4. We have the following.

(i) If $m>0$, we have $(\mathcal{L} f)_{\beta}^{m} \in \mathbf{T}_{\text {hol }}^{m}\left(\beta \in K_{\mathbf{A}}^{\times}\right)$.

(ii) If $m<0$, we have $(\mathcal{L} f)^{m}=0$.

Proof. By (7.2), we have $(\mathcal{L} f)_{\beta}^{m} \in \mathbf{T}^{m, 0}$. Suppose that $m>0$. By Lemma 4.2(i), we have $\mathbf{T}^{m, 0}=\bigoplus_{\chi \in \mathcal{X}, w_{\infty}(\chi) \leqslant-1} \mathbf{T}_{\chi}^{m, 0}$. On the other hand, Propositions 8.4 and 9.3 imply that $(\mathcal{L} f)_{\beta}^{m} \in$ $\bigoplus_{\chi \in \mathcal{X}, w_{\infty}(\chi) \geqslant-1} \mathbf{T}_{\chi}^{m, 0}$. We thus have $(\mathcal{L} f)_{\beta}^{m} \in \bigoplus_{\chi \in \mathcal{X}, w_{\infty}(\chi)=-1} \mathbf{T}_{\chi}^{m, 0}=\mathbf{T}_{\text {hol }}^{m}$ (see Lemma 4.2(ii)). The assertion (ii) can be proved similarly. 


\section{On the Fourier-JACOBI EXPANSION OF THE UNITARY KudLA LIFT}

\subsection{Calculation of local integrals at $\infty$}

From now on, we assume that $m>0$. Let $\Theta \in \mathbf{T}_{\text {hol }}^{m}$ and fix an $r_{f} \in R_{\mathbf{A}, f}$. We put $\tau\left(r_{\infty}\right)=I_{\Theta}\left(r_{f} r_{\infty}\right)$ for $r_{\infty} \in R_{\infty}$.

Lemma 9.5. For $z \in \mathfrak{H}$, we have

$$
\int_{\mathbb{C}} \mathbf{e}[z \mathrm{~N}(w)] \tau\left(w, x_{w}\right) d w=\frac{\sqrt{-1}}{z+m \theta} \tau(1) .
$$

Proof. Since

$$
w \mapsto \widetilde{\tau}(w):=\mathbf{e}\left[-\frac{m \kappa}{2} \mathrm{~N}(w)\right] \tau(w, 0)
$$

is holomorphic on $\mathbb{C}$, the integral of the lemma is equal to

$$
\int_{\mathbb{C}} \mathbf{e}[(z+m \theta) \mathrm{N}(w)] \widetilde{\tau}(w) d w=\widetilde{\tau}(0) \int_{\mathbb{C}} \mathbf{e}[(z+m \theta) \mathrm{N}(w)] d w=\frac{\sqrt{-1}}{z+m \theta} \tau(1)
$$

and we are done.

For $\beta_{\infty} \in K_{\infty}^{\times}$and $W \in \mathcal{W}_{\infty}^{m}(0)$, set

$$
\begin{aligned}
& \mathcal{I}_{\beta_{\infty}}(W, \tau) \\
& \quad=\mathrm{N}\left(\beta_{\infty}\right) \int_{H_{\eta_{m}, \infty} \backslash H_{\infty}} \int_{\mathbb{C}} \chi_{0}^{-1}(\operatorname{det} h) \Phi_{0, \infty}\left(\beta_{\infty} h^{-1} \eta_{m}\right)\left(\mathcal{M}_{\chi_{0}}^{T}(h) \varphi_{0, \infty}\right)(-w) W(h) \overline{\tau\left(w, x_{w}\right)} d w d h .
\end{aligned}
$$

Here we note that

$$
h \mapsto \chi_{0}^{-1}(\operatorname{det} h) \Phi_{0, \infty}\left(\beta_{\infty} h^{-1} \eta_{m}\right) W(h) \int_{\mathbb{C}}\left(\mathcal{M}_{\chi_{0}}^{T}(h) \varphi_{0, \infty}\right)(-w) \overline{\tau\left(w, x_{w}\right)} d w
$$

is left $H_{\eta_{m}, \infty}$-invariant. This fact is verified by using Lemma 8.3 and the fact that $\tau$ is left $K_{\infty}^{1}$-invariant.

Proposition 9.6. We have

$$
\mathcal{I}_{\beta_{\infty}}(W, \tau)=c_{\infty} \cdot \beta_{\infty}^{l} \mathbf{e}_{m}\left[\frac{\kappa}{2}\left(\mathrm{~N}\left(\beta_{\infty}\right)-1\right)\right] \overline{\tau(1)} W\left(h_{m \theta, \infty}\right)
$$

(for the definitions of $c_{\infty}$ and $h_{m \theta, \infty}$, see $\S 7.6$ ).

Proof. To simplify the notation, we suppress $\infty$ from the notation. Since $H_{\eta_{m}}$ is compact and $\operatorname{vol}\left(H_{\eta_{m}}\right)=1, \mathcal{I}_{\beta}(W, \tau)$ is equal to

$$
\mathrm{N}(\beta) \int_{H} \int_{\mathbb{C}} \chi_{0}^{-1}(\operatorname{det} h) \Phi_{0, \infty}\left(\beta h^{-1} \eta_{m}\right)\left(\mathcal{M}_{\chi_{0}}^{T}(h) \varphi_{0, \infty}\right)(-w) W(h) \overline{\tau\left(w, x_{w}\right)} d w d h .
$$

Observe that the integrand of the above integral is, as a function of $h$, right $\mathcal{U}_{\infty}$-invariant, since $\Phi_{0, \infty}\left(u^{-1} Z\right)=j^{\prime}\left(u, z_{0}\right)^{l} \Phi_{0, \infty}(Z)$ and $\mathcal{M}_{\chi_{0}}^{T}(u) \varphi_{0, \infty}=j\left(u, z_{0}\right)^{-1} \varphi_{0, \infty}$ for $u \in \mathcal{U}_{\infty}$. Thus, $\mathcal{I}_{\beta}(W, \tau)$ is equal to

$$
\begin{aligned}
2 \pi \mathrm{N} & (\beta) \int_{\mathbb{R}} \int_{0}^{\infty} \int_{\mathbb{C}} y^{-1} \Phi_{0, \infty}\left(\beta \mathbf{d}_{H}\left(\sqrt{y}^{-1}\right) \mathbf{n}_{H}(-x) \eta_{m}\right) \\
& \times\left(\mathcal{M}_{\chi_{0}}^{T}\left(\mathbf{n}_{H}(x) \mathbf{d}_{H}(\sqrt{y})\right) \varphi_{0}\right)(-w) W\left(\mathbf{n}_{H}(x) \mathbf{d}_{H}(\sqrt{y})\right) \overline{\tau\left(w, x_{w}\right)} d w d^{\times} y d x \\
= & 2 \pi \mathrm{N}(\beta) \beta^{l} \int_{\mathbb{R}} \int_{0}^{\infty} \int_{\mathbb{C}} y^{-1}\left\{\sqrt{y}^{-1}\left(-x+m \theta^{\sigma}\right)-\sqrt{-1} \sqrt{y}\right\}^{l} \\
& \times \mathbf{e}\left[\frac{\sqrt{-1}}{2} \mathrm{~N}(\beta)\left\{y+y^{-1} \mathrm{~N}\left(-x+m \theta^{\sigma}\right)\right\}\right] \cdot y^{1 / 2} \mathbf{e}[(x+\sqrt{-1} y) \mathrm{N}(w)] \\
& \times y^{(l-1) / 2} \widetilde{W}(x+\sqrt{-1} y) \overline{\tau\left(w, x_{w}\right)} d w d^{\times} y d x .
\end{aligned}
$$




\section{A. Murase And T. Sugano}

By Lemma 9.2, we have $\widetilde{W}(x+\sqrt{-1} y)=(m \kappa)^{l-1} \widetilde{W}(m \theta) \cdot\left(x+\sqrt{-1} y-m \theta^{\sigma}\right)^{-l+1}$ and, hence,

$$
\begin{aligned}
\mathcal{I}_{\beta}(W, \tau)= & 2 \pi \mathrm{N}(\beta) \beta^{l}(m \kappa)^{l-1} \widetilde{W}(m \theta) \int_{\mathbb{R}} \int_{0}^{\infty} y^{-1}\left(x+\sqrt{-1} y-m \theta^{\sigma}\right) \\
& \times \mathbf{e}\left[\frac{\sqrt{-1}}{2} \mathrm{~N}(\beta)\left\{y+y^{-1} \mathrm{~N}\left(-x+m \theta^{\sigma}\right)\right\}\right] \int_{\mathbb{C}} \mathbf{e}[(x+\sqrt{-1} y) \mathrm{N}(w)] \overline{\tau\left(w, x_{w}\right)} d w d^{\times} y d x .
\end{aligned}
$$

Using Lemma 9.5, we obtain

$$
\mathcal{I}_{\beta}(W, \tau)=2 \pi \sqrt{-1}(m \kappa)^{l-1} \mathrm{~N}(\beta) \beta^{l} \cdot \overline{\tau(1)} \widetilde{W}(m \theta) \cdot I,
$$

where

$$
I=\int_{\mathbb{R}} \int_{0}^{\infty} y^{-1} \mathbf{e}\left[\frac{\sqrt{-1}}{2} \mathrm{~N}(\beta)\left(y+y^{-1} \mathrm{~N}\left(-x+m \theta^{\sigma}\right)\right)\right] d^{\times} y d x=\left(\frac{m \mathrm{~N}(\beta)|\kappa|}{2}\right)^{-1} \mathbf{e}_{m}\left[\frac{\kappa}{2} \mathrm{~N}(\beta)\right] .
$$

Since $\widetilde{W}(m \theta)=j^{\prime}\left(h_{m \theta, \infty}, z_{0}\right)^{l-1} W\left(h_{m \theta, \infty}\right)$, we have

$$
\mathcal{I}_{\beta}(W, \tau)=2 \pi \sqrt{-1}(m \kappa)^{l-1}(m|\kappa| / 2)^{-1} j^{\prime}\left(h_{m \theta, \infty}, z_{0}\right)^{l-1} \mathbf{e}[m \kappa / 2] \beta^{l} \mathbf{e}_{m}\left[\frac{\kappa}{2}(\mathrm{~N}(\beta)-1)\right] \overline{\tau(1)} W\left(h_{m \theta, \infty}\right),
$$

which proves the proposition.

By Propositions 8.4 and 9.6, we have proved the following result, from which Proposition 7.3 follows.

Proposition 9.7. For $m>0, \beta=\beta_{f} \beta_{\infty} \in K_{\mathbf{A}}^{\times}$and $\Theta \in \mathbf{T}_{\text {hol }}^{m}$, we have

$$
\begin{aligned}
& \left\langle\left\langle(\mathcal{L} f)_{\beta}^{m}, \Theta\right\rangle\right\rangle_{R} \\
& =c_{\infty} \cdot \beta_{\infty}^{l} \mathbf{e}_{m}\left[\frac{\kappa}{2}\left(\mathrm{~N}\left(\beta_{\infty}\right)-1\right)\right]\left|\mathrm{N}\left(\beta_{f}\right)\right|_{\mathbf{A}} \int_{\left(H_{\eta_{m}}\right)_{\mathbf{A}, f} \backslash H_{\mathbf{A}, f}} \chi_{0}^{-1}\left(\operatorname{det} h_{f}\right) \Phi_{0, f}\left(\beta_{f} h_{f}^{-1} \eta_{m}\right) W_{\chi, f}^{m}\left(h_{f} h_{m \theta, \infty}\right) \\
& \quad \times \int_{K_{\mathbf{A}, f}}\left(\mathcal{M}_{\chi_{0}}^{T}\left(h_{f}\right) \varphi_{0, f}\right)(-w) \overline{I_{\Theta}\left(w, x_{w}\right)} d w d h_{f} .
\end{aligned}
$$

\section{Local integrals at finite primes}

\subsection{Local integrals}

In this section, we reduce the calculation of the Fourier-Jacobi components of $\mathcal{L} f$ to that of certain local integrals. Until the end of the paper, we fix a finite prime $p$ and often suppress the subscript $p$. We write $F$ and $K$ for $\mathbb{Q}_{p}$ and $K_{p}$, respectively. Let $\operatorname{ord}_{F}: F^{\times} \rightarrow \mathbb{Z}$ be the additive valuation of $F$ normalized by $\operatorname{ord}_{F}(\pi)=1$. Let $\mathfrak{p}_{F}=\pi \mathcal{O}_{F}$ be the maximal ideal of $\mathcal{O}_{F}$ and put $\delta=\operatorname{ord}_{F} D$. We denote by $\mathcal{X}$ the set of characters of $K^{\times}$with $\left.\chi\right|_{F^{\times}}=\omega_{K / F}$. Let $\mathcal{E}$ be the set of $(m, \mathfrak{a}, \chi)$, where $m \in F^{\times}, \mathfrak{a}$ is an ideal of $K$ with $\mu:=\operatorname{ord}_{F}(m \mathrm{~N}(\mathfrak{a})) \geqslant 0$ and $\chi \in \mathcal{X}$ satisfying the following two conditions:

(i) $A(\chi)=A_{p}(\chi)= \begin{cases}\mu & \text { if } \delta=0, \\ 2(\mu+\delta) & \text { if } \delta>0 \text { and } \mu>0, \\ 2 \delta \text { or } 2 \delta-1 & \text { if } \delta>0 \text { and } \mu=0,\end{cases}$

where $A_{p}(\chi)$ is defined in $\S 4.7$;

(ii) $\epsilon\left(1 / 2, \chi, \psi_{m, K}\right)=\chi\left(\kappa^{-1}\right)$.

Let $X_{\text {unr }}\left(K^{1}\right)$ be the group of unitary characters $\Omega$ of $K^{1}=\left\{t \in K^{\times} \mid t t^{\sigma}=1\right\}$ with $\left.\Omega\right|_{\mathcal{O}_{K}^{1}}=1$.

For $\chi \in \mathcal{X}$, let $\mathcal{W}^{m}(\chi)$ be the space of $\mathbb{C}$-valued smooth functions $W$ on $H$ satisfying

$$
W\left(\iota_{m}(t) h u\right)=\left(\chi / \chi_{0}\right)^{1}(t) \tilde{\chi}_{0}(u) W(h)
$$




\section{ON THE Fourier-JACOBI EXPANSION OF THE UNITARY KUDLA LIFT}

for $t \in K^{1}, h \in H, u \in \mathcal{U}^{\prime}=\left\{\left(\begin{array}{ll}a & b \\ c & d\end{array}\right) \in \mathcal{U} \mid c \in D \mathcal{O}_{K}\right\}$ (for the definition of $\iota_{m}$, see (7.6)). For $\Omega \in X_{\text {unr }}\left(K^{1}\right)$, let $\mathcal{W}^{m}\left(\chi ; \chi_{0} \Omega\right)$ be the space of $W \in \mathcal{W}^{m}(\chi)$ satisfying $W\left(t 1_{2} \cdot h\right)=\left(\chi_{0} \Omega\right)(t) W(h)$ for $t \in K^{1}$.

For $(m, \mathfrak{a}, \chi) \in \mathcal{E}$, let $\mathcal{T}^{m}(\mathfrak{a}, \chi)$ be the space consisting of $\mathbb{C}$-valued functions $\tau$ on $R$ satisfying the following four conditions.

(10.2) We have $\tau\left(\operatorname{trr}_{0}(0, x)\right)=\psi_{m}(x) \tau(r)\left(t \in K^{1}, r \in R, r_{0} \in R(\mathfrak{a}), x \in F\right)$.

(10.3) If $\mathfrak{a}^{\prime}$ is an ideal of $K$ with $\mathfrak{a}^{\prime} \supsetneqq \mathfrak{a}$, then

$$
\int_{N\left(\mathfrak{a}^{\prime}\right)} \tau(r n) d n=0 .
$$

(Recall that $\left.N\left(\mathfrak{a}^{\prime}\right)=\left\{\left(w, x+x_{w}\right) \in N \mid w \in \mathfrak{a}^{\prime}, x \in \mathfrak{a}^{\prime} \mathfrak{a}^{\prime \sigma}\right\}.\right)$

(10.4) Suppose that $K / F$ is ramified. Then

$$
\int_{\Pi^{-1} \mathfrak{a}} \tau\left(r\left(w, x_{w}\right)\right) d_{\Pi^{-1} \mathfrak{a}} w= \begin{cases}0 & \text { if } \chi \text { is ordinary } \\ \tau(r) & \text { if } \chi \text { is exceptional. }\end{cases}
$$

(For the definition of $d_{\Pi^{-1} \mathfrak{a}} w$, see $\S 2.4$.)

(10.5) There exists a lattice $L_{0}$ of $K$ such that, for any lattice $L$ of $K$ with $L \supset L_{0}$, we have

$$
\tau(r t)=\lambda_{K}\left(\psi_{m}\right)^{-1} \chi\left(\frac{1-t}{\kappa}\right)|\mathrm{N}(1-t)|_{F}^{1 / 2} \int_{L} \psi_{m}\left(\frac{1}{2}\langle w, t w\rangle_{\kappa}\right) \tau(r((1-t) w, 0)) d^{m} w
$$

for $t \in K^{1}-\{1\}$.

Let $(m, \mathfrak{a}, \chi) \in \mathcal{E}$ and $\mathfrak{b}$ be an ideal of $K$. For $W \in \mathcal{W}^{m}(\chi)$ and $\tau \in \mathcal{T}^{m}(\mathfrak{a}, \chi)$, we set

$$
\mathcal{I}_{\mathfrak{b}}(W, \tau)=|\mathrm{N}(\beta)|_{F} \int_{H_{\eta_{m} \backslash H}} \int_{K} \chi_{0}^{-1}(\operatorname{det} h) \Phi_{0}\left(\beta h^{-1} \eta_{m}\right)\left(\mathcal{M}_{\chi_{0}}^{T}(h) \varphi_{0}\right)(-w) W(h) \overline{\tau\left(w, x_{w}\right)} d w d h,
$$

where $\beta$ is an element of $K^{\times}$such that $\mathfrak{b}=\beta \mathcal{O}_{K}$. Note that the right-hand side of $I_{\mathfrak{b}}(W, \tau)$ does not depend on the choice of $\beta$.

Remark. Going back to the global situation, let $(m, \mathfrak{a}, \chi) \in \mathcal{E}, f \in S_{l-1}\left(\mathcal{U}_{f}^{\prime}, \widetilde{\chi}_{0}\right)$ and $\Theta \in$ $\mathbf{T}_{\text {hol,prim }}^{m}(\mathfrak{a}, \chi)$ (cf. $\S 7.6$ ). Then, for every $p$, the restriction of $W_{\chi, f}^{m}$ to $H_{p}$ belongs to $\mathcal{W}^{m}\left(\chi_{p}\right)$. If $f \in S_{l-1}\left(\mathcal{U}_{f}^{\prime}, \tilde{\chi}_{0} ; \chi_{0} \Omega\right)$, we have $\left.W_{\chi, f}^{m}\right|_{H_{p}} \in \mathcal{W}^{m}\left(\chi_{p} ;\left(\chi_{0} \Omega\right)_{p}\right)$. We also note that the restriction of $I_{\Theta}$ to $R_{p}$ belongs to $\mathcal{T}^{m}\left(\mathfrak{a}_{p}, \chi_{p}\right)$.

\subsection{Local main results}

The proofs of Theorems 7.5 and 7.6 are reduced to those of the following results.

Theorem 10.1. Let $(m, \mathfrak{a}, \chi) \in \mathcal{E}$. Then, for $W \in \mathcal{W}^{m}(\chi)$ and $\tau \in \mathcal{T}^{m}(\mathfrak{a}, \chi)$, we have

$$
\begin{aligned}
\mathcal{I}_{\mathfrak{a}}(W, \tau)= & \chi_{0}^{-1}(\alpha)|\mathrm{N}(\alpha)|_{F}^{3 / 2}|D|_{F}^{1 / 2} v_{0} \cdot \overline{\tau(1)} \\
& \times\left\{W\left(\mathbf{d}_{H}\left(\alpha^{-1}\right)\right)+\delta_{e}(\chi) \cdot p^{-1 / 2} \chi^{-1}(m \theta) W\left(\mathbf{d}_{H}\left(\alpha^{-1}\right) w_{0}\right)\right\} .
\end{aligned}
$$

Here $\alpha$ is an element of $K^{\times}$with $\mathfrak{a}=\alpha \mathcal{O}_{K}$,

$$
v_{0}=p^{\mu} \times \begin{cases}1 & \text { if } \delta=0 \text { and } \mu=0, \\ 1-\omega_{K / F}(p) p^{-1} & \text { if } \delta=0 \text { and } \mu>0, \\ \frac{2 p}{1+p} & \text { if } \delta>0\end{cases}
$$




\section{A. Murase And T. Sugano}

and

$$
\delta_{e}(\chi)= \begin{cases}1 & \text { if } p \mid D \text { and } \chi \text { is exceptional } \\ 0 & \text { otherwise }\end{cases}
$$

Theorem 10.2. Let $(m, \mathfrak{a}, \chi) \in \mathcal{E}$ and $\Omega \in X_{\text {unr }}\left(K^{1}\right)$. For $W \in \mathcal{W}^{m}\left(\chi ; \chi_{0} \Omega\right)$ and $\tau \in \mathcal{T}^{m}(\mathfrak{a}, \chi)$, we have the following.

(i) If $K / F$ is an unramified quadratic extension, we have

$$
\begin{aligned}
& p^{2} \mathcal{I}_{p^{k+1} \mathfrak{a}}(W, \tau)-p^{-1} \mathcal{I}_{p^{k} \mathfrak{a}}\left(W * \phi_{1}, \tau\right) \\
& \quad+\left\{\delta(k+\mu>0) p^{-1}-1\right\} \mathcal{I}_{p^{k} \mathfrak{a}}(W, \tau)+p^{-2} \mathcal{I}_{p^{k-1} \mathfrak{a}}(W, \tau)=0
\end{aligned}
$$

for $k \geqslant 0$.

(ii) If $K / F$ is a ramified quadratic extension, we have

$$
\begin{aligned}
& p \mathcal{I}_{\Pi^{k+1} \mathfrak{a}}(W, \tau)-p^{-1 / 2} \mathcal{I}_{\Pi^{k} \mathfrak{a}}\left(W *\left(\phi^{+}+\phi^{-}\right), \tau\right) \\
& \quad-\delta(k=0 \text { and } \chi \text { is ordinary }) \mathcal{I}_{\Pi^{k} \mathfrak{a}}(W, \tau)+p^{-1} \mathcal{I}_{\Pi^{k-1} \mathfrak{a}}(W, \tau)=0
\end{aligned}
$$

for $k \geqslant 0$.

(iii) If $K=F \oplus F$, we have

$$
\begin{aligned}
& p \mathcal{I}_{\Pi_{1}^{k_{1}+1} \Pi_{2}^{k_{2} \mathfrak{a}}}(W, \tau)-p^{-1 / 2} \mathcal{I}_{\Pi_{1}^{k_{1}} \Pi_{2}^{k_{2}} \mathfrak{a}}\left(W * \phi_{1}, \tau\right) \\
& \quad-\mathcal{I}_{\Pi_{1}^{k_{1}} \Pi_{2}^{k_{2} \mathfrak{a}}}(W, \tau)+\mathcal{I}_{\Pi_{1}^{k_{1}-1} \Pi_{2}^{k_{2}+1} \mathfrak{a}}\left(W * \phi_{0}^{+}, \tau\right)+p^{-1} \mathcal{I}_{\Pi_{1}^{k_{1}} \Pi_{2}^{k_{2}-1} \mathfrak{a}}(W, \tau)=0
\end{aligned}
$$

and

$$
\begin{aligned}
& p \mathcal{I}_{\Pi_{1}^{k_{1}} \Pi_{2}^{k_{2}+1} \mathfrak{a}}(W, \tau)-p^{-1 / 2} \mathcal{I}_{\Pi_{1}^{k_{1}} \Pi_{2}^{k_{2}} \mathfrak{a}}\left(W * \phi_{2}, \tau\right) \\
& \quad-\mathcal{I}_{\Pi_{1}^{k_{1}} \Pi_{2}^{k_{2} \mathfrak{a}}}(W, \tau)+\mathcal{I}_{\Pi_{1}^{k_{1}+1} \Pi_{2}^{k_{2}-1} \mathfrak{a}}\left(W * \phi_{0}^{-}, \tau\right)+p^{-1} \mathcal{I}_{\Pi_{1}^{k_{1}-1} \Pi_{2}^{k_{2}}}(W, \tau)=0
\end{aligned}
$$

for $k_{1}, k_{2} \geqslant 0$.

10.3 We close this section with an elementary fact, which is needed in the proof of Theorem 10.2. Let $C^{\infty}\left(H / \mathcal{U}^{\prime}, \widetilde{\chi}_{0}\right)$ be the space of smooth functions $f$ on $H$ satisfying $f(h u)=\widetilde{\chi}_{0}(u) f(h)(h \in H$, $\left.u \in \mathcal{U}^{\prime}\right)$. Then the Hecke algebra $\mathcal{H}^{H}(\operatorname{cf} . \S 3.4)$ acts on $C^{\infty}\left(H / \mathcal{U}^{\prime}, \tilde{\chi}_{0}\right)$ on the right in a natural manner. For $\phi \in \mathcal{H}^{H}$, put $\phi^{\vee}(h)=\chi_{0}(\operatorname{det} h) \phi\left(h^{-1}\right)$. Note that $\phi^{\vee}$ is also in $\mathcal{H}^{H}$.

Lemma 10.3. For $f, f^{\prime} \in C^{\infty}\left(H / \mathcal{U}^{\prime}, \widetilde{\chi}_{0}\right)$ and $\phi \in \mathcal{H}^{H}$, we have

$$
\int_{H} \chi_{0}^{-1}(\operatorname{det} h)(f * \phi)(h) f^{\prime}(h) d h=\int_{H} \chi_{0}^{-1}(\operatorname{det} h) f(h)\left(f^{\prime} * \phi^{\vee}\right)(h) d h .
$$

\section{Calculation of local integrals at finite primes: primitive case}

11.1 Until the end of the paper, we keep the notation of $\S 10$, and let $(m, \mathfrak{a}, \chi) \in \mathcal{E}, W \in \mathcal{W}^{m}(\chi)$ and $\tau \in \mathcal{T}^{m}(\mathfrak{a}, \chi)$. Take and fix an $\alpha \in K^{\times}$such that $\mathfrak{a}=\alpha \mathcal{O}_{K}$. To simplify the notation, we write $\eta$ and $\iota$ for $\eta_{m}$ and $\iota_{m}$, respectively. We also write $\mathbf{d}(a), \mathbf{n}(x)$ and $\overline{\mathbf{n}}(x)$ for $\mathbf{d}_{H}(a), \mathbf{n}_{H}(x)$ and $\overline{\mathbf{n}}_{H}(x)$ respectively, if there is no fear of confusion. Let $H_{\eta}=\{h \in H \mid h \eta=\eta\}$ and put $L_{k}=\mathcal{O}_{F}+\theta \mathfrak{p}_{F}^{k}$ for $k \in \mathbb{Z}, k \geqslant 0$. In this section, we prove Theorem 10.1 .

\subsection{Inert case}

In this subsection, we consider the case where $K / F$ is inert. Note that $\mathcal{U}=\mathcal{U}^{\prime}$ in this case. Set $h_{l}=\mathbf{d}\left(\pi^{-l} \alpha^{-1}\right) \quad(l \in \mathbb{Z})$. We need the following Cartan-type decomposition of $H$. 


\section{ON THE Fourier-JACOBI EXPANSION OF THE UNITARY KUDLA LIFT}

LEMma 11.1. We have the following.

(i) We have $H=\bigcup_{l \geqslant-[\mu / 2]} H_{\eta} h_{l} \mathcal{U}$ (disjoint union).

(ii) For $l \in \mathbb{Z}$ with $l \geqslant-[\mu / 2]$, we have

$$
v_{l}:=\operatorname{vol}\left(H_{\eta} h_{l} \mathcal{U}\right)=p^{2 l+\mu} \times \begin{cases}1 & \text { if } 2 l+\mu=0, \\ 0 & \text { if } 2 l+\mu>0 .\end{cases}
$$

Proof. The first assertion is easily verified. To prove the second, observe that

$$
H_{\eta} h_{l} \mathcal{U}=\bigcup_{z \in \mathcal{O}_{K}^{\times} /\left(\mathcal{O}_{K}^{\times} \cap L_{2 l+\mu}\right)} \iota\left(z^{\sigma} / z\right) h_{l} \mathcal{U} \quad \text { (disjoint union). }
$$

Since $\left[\mathcal{O}_{K}^{\times}:\left(\mathcal{O}_{K}^{\times} \cap L_{k}\right)\right]$ is equal to $p^{k}\left(1+p^{-1}\right)$ if $k \geqslant 1$ and 1 if $k=0$, we are done.

Lemma 11.2. For $W \in \mathcal{W}^{m}(\chi)$, we have $\operatorname{Supp}(W) \subset \bigcup_{l \geqslant 0} H_{\eta} h_{l} \mathcal{U}$.

Proof. There is nothing to prove if $\mu \leqslant 1$. Assume that $\mu \geqslant 2$ and $-[\mu / 2] \leqslant l<0$. It is sufficient to show that $W\left(h_{l}\right)=0$. Since $A(\chi)=\mu$, there exists an $x \in \mathcal{O}_{F}$ such that $\chi\left(1+\pi^{\mu-1} x \theta\right) \neq 1$. Put

$$
t_{x}=\frac{1+\pi^{\mu-1} x \theta^{\sigma}}{1+\pi^{\mu-1} x \theta} \in K^{1}
$$

Then $\iota\left(t_{x}\right) h_{l}=h_{l} u$, where

$$
u=\frac{1}{1+\pi^{\mu-1} x \theta}\left(\begin{array}{cc}
1 & m \mathrm{~N}(\alpha) \pi^{\mu+2 l-1} \mathrm{~N}(\theta) x \\
-(m \mathrm{~N}(\alpha))^{-1} \pi^{\mu-2 l-1} x & 1+\operatorname{Tr}(\theta) \pi^{\mu-1} x
\end{array}\right) .
$$

Since $u=\left(u_{i j}\right) \in \mathcal{U}$ and $u_{21} \in \pi \mathcal{O}_{K}$, we have

$$
\left(\chi / \chi_{0}\right)\left(1+\pi^{\mu-1} x \theta\right) W\left(h_{l}\right)=W\left(\iota\left(t_{x}\right) h_{l}\right)=W\left(h_{l} u\right)=\tilde{\chi}_{0}(u) W\left(h_{l}\right)=\chi_{0}^{-1}\left(1+\pi^{\mu-1} x \theta\right) W\left(h_{l}\right) .
$$

This implies that $W\left(h_{l}\right)=0$.

In view of Lemmas 11.1 and $11.2, \mathcal{I}_{\mathfrak{a}}(W, \tau)$ is equal to

$$
\begin{aligned}
& |\mathrm{N}(\alpha)|_{F} \sum_{l=0}^{\infty} v_{l} W\left(h_{l}\right) \chi_{0}\left(\alpha^{\sigma} / \alpha\right) \Phi_{0}\left(\begin{array}{c}
\pi^{l} m \mathrm{~N}(\alpha) \theta^{\sigma} \\
\pi^{-l}
\end{array}\right) \chi_{0}\left(\pi^{l} \alpha\right)\left|\mathrm{N}\left(\pi^{-l} \alpha^{-1}\right)\right|_{F}^{1 / 2} \int_{K} \varphi_{0}\left(\pi^{-l} \alpha^{-1} w\right) \overline{\tau\left(w, x_{w}\right)} d w \\
& \quad=v_{0} \chi_{0}^{-1}(\alpha)|\mathrm{N}(\alpha)|_{F}^{1 / 2} W\left(h_{0}\right) \int_{\mathfrak{a}} \overline{\tau\left(w, x_{w}\right)} d w \\
& \quad=v_{0} \chi_{0}^{-1}(\alpha)|\mathrm{N}(\alpha)|_{F}^{3 / 2} W\left(\mathbf{d}\left(\alpha^{-1}\right) \overline{\tau(1)} .\right.
\end{aligned}
$$

This completes the proof of Theorem 10.1 in the inert case.

\subsection{Ramified case}

In this subsection, we consider the case where $K / F$ is a ramified quadratic extension. Recall that $\theta$ is a prime element of $K$. Set $h_{l}=\mathbf{d}\left(\Pi^{-l} \alpha^{-1}\right)$ for $l \in \mathbb{Z}$.

LEMma 11.3. We have the following.

(i) We have

$$
H=\bigcup_{l \geqslant-\mu} H_{\eta} h_{l} \mathcal{U}^{\prime} \cup \bigcup_{l \geqslant-\mu} \bigcup_{a \in \mathfrak{p}_{F} / D \mathcal{O}_{F}} H_{\eta} h_{l} \mathbf{n}(a) w_{0} \mathcal{U}^{\prime}
$$

where $w_{0}=\left(\begin{array}{rr}0 & 1 \\ -1 & 0\end{array}\right)$.

(ii) We have $H_{\eta} h_{-\mu} \mathbf{n}(a) w_{0} \mathcal{U}^{\prime}=H_{\eta} h_{-\mu} w_{0} \mathcal{U}^{\prime}$ for $a \in \mathfrak{p}_{F}$. 


\section{A. Murase And T. Sugano}

Proof. For $h, h^{\prime} \in H$, we write $h \sim h^{\prime}$ if $h^{\prime} \in H_{\eta} h \mathcal{U}^{\prime}$. It is easily verified that $H=\bigcup_{l \geqslant-\mu} H_{\eta} h_{l} \mathcal{U}$ and $H_{\eta} h_{l} \mathcal{U}=\left\{h \in H \mid \Pi^{l} \alpha \cdot h^{-1} \eta \in \mathcal{O}_{K}^{2}-\Pi_{\mathcal{O}_{K}^{2}}^{2}\right.$. Let $h=h_{l} u\left(l \geqslant-\mu, u=\left(u_{i j}\right) \in \mathcal{U}\right)$. To prove part (i), it suffices to show that

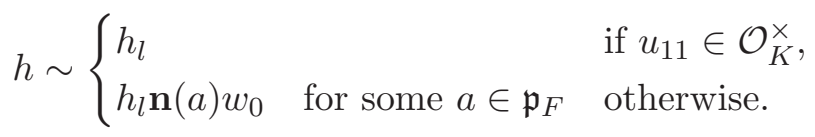

First suppose that $u_{11} \in \mathcal{O}_{K}^{\times}$. Then $h \sim h_{l} \overline{\mathbf{n}}(c)$ with $c=u_{11}^{-1} u_{21} \in \mathcal{O}_{F}$. If we put

$$
z=1+y \theta \quad \text { with } y=\frac{c m \mathrm{~N}(\alpha) \pi^{l}}{1-c \operatorname{Tr}(\theta) m \mathrm{~N}(\alpha) \pi^{l}} \in \mathcal{O}_{F},
$$

we have

$$
h \sim \iota\left(z^{\sigma} / z\right) h_{l} \overline{\mathbf{n}}(c) h_{l} \frac{1}{z}\left(\begin{array}{cc}
1+c m \mathrm{~N}(\alpha \theta) \pi^{l} y & m \mathrm{~N}(\alpha \theta) \pi^{l} y \\
0 & 1+\operatorname{Tr}(\theta) y
\end{array}\right) \sim h_{l} .
$$

Next suppose that $u_{11} \in \Pi \mathcal{O}_{K}$ (and, hence, $\left.u_{21} \in \mathcal{O}_{K}^{\times}\right)$. Then $h \mathbf{n}\left(-u_{21}^{-1} u_{22}\right) \mathbf{d}\left(-\left(u_{21}^{\sigma}\right)^{-1}\right)=h_{l} \mathbf{n}(a) w_{0}$ with $a=u_{11} u_{21}^{-1} \in \mathfrak{p}_{F}$, which completes the proof of (11.1). To prove part (ii), let $a \in \mathfrak{p}_{F}$ and put

$$
z=1+y \theta \quad \text { with } y=-\frac{a \pi^{\mu}}{m \mathrm{~N}(\alpha \theta)} .
$$

We then have $y \in \mathcal{O}_{F}$ and

$$
\iota\left(z^{\sigma} / z\right) h_{-\mu} \mathbf{n}(a) w_{0}=h_{-\mu} w_{0}\left(\begin{array}{cc}
z^{\sigma} & m^{-1} \mathrm{~N}(\alpha)^{-1} \pi^{\mu} y z^{-1} \\
0 & z^{-1}
\end{array}\right),
$$

which implies $h_{-\mu} \mathbf{n}(a) w_{0} \sim h_{-\mu} w_{0}$.

LEMma 11.4. We have the following.

(i) For $l \geqslant-\mu$, we have

(ii) We have

$$
\operatorname{vol}\left(H_{\eta} h_{l} \mathcal{U}^{\prime}\right)=\frac{2 p}{1+p} p^{l+\mu}
$$

$$
\operatorname{vol}\left(H_{\eta} h_{-\mu} w_{0} \mathcal{U}^{\prime}\right)=\frac{2}{1+p}
$$

Proof. It is easily verified that

$$
H_{\eta} h_{l} \mathcal{U}^{\prime}=\bigcup_{z \in \mathcal{O}_{K}^{\times} /\left(\mathcal{O}_{K}^{\times} \cap L_{\mu+l+\delta}\right)}\left(\iota\left(z^{\sigma} / z\right) h_{l} \mathcal{U}^{\prime} \cup \iota\left((\Pi z)^{\sigma} /(\Pi z)\right) h_{l} \mathcal{U}^{\prime}\right) \quad \text { (disjoint union) }
$$

for $l \geqslant-\mu$, and

$$
H_{\eta} h_{-\mu} w_{0} \mathcal{U}^{\prime}=\bigcup_{z \in \mathcal{O}_{K}^{\times} /\left(\mathcal{O}_{K}^{\times} \cap L_{\delta-1}\right)}\left(\iota\left(z^{\sigma} / z\right) h_{-\mu} w_{0} \mathcal{U}^{\prime} \cup \iota\left((\Pi z)^{\sigma} /(\Pi z)\right) h_{-\mu} w_{0} \mathcal{U}^{\prime}\right) \quad \text { (disjoint union). }
$$

The lemma now follows from $\left[\mathcal{O}_{K}^{\times}:\left(\mathcal{O}_{K}^{\times} \cap L_{k}\right)\right]=p^{k}(k \geqslant 0)$ and $\left[\mathcal{U}: \mathcal{U}^{\prime}\right]=p^{\delta}\left(1+p^{-1}\right)$.

Lemma 11.5. Let $W \in \mathcal{W}^{m}(\chi)$.

(i) If $\chi$ is ordinary, we have

$$
\operatorname{Supp}(W) \subset \bigcup_{l \geqslant 0} H_{\eta} h_{l} \mathcal{U}^{\prime} \cup \bigcup_{l \geqslant 1} \bigcup_{a \in \mathfrak{p}_{F} / D \mathcal{O}_{F}} H_{\eta} h_{l} \mathbf{n}(a) w_{0} \mathcal{U}^{\prime} .
$$

(ii) If $\chi$ is exceptional, we have

$$
\operatorname{Supp}(W) \subset \bigcup_{l \geqslant 0} H_{\eta} h_{l} \mathcal{U}^{\prime} \cup \bigcup_{l \geqslant 1} \bigcup_{a \in \mathfrak{p}_{F} / D \mathcal{O}_{F}} H_{\eta} h_{l} \mathbf{n}(a) w_{0} \mathcal{U}^{\prime} \cup H_{\eta} h_{0} w_{0} \mathcal{U}^{\prime}
$$




\section{ON THE Fourier-JACOBI EXPANSION OF THE UNITARY KUDLA LIFT}

Proof. There is nothing to prove when $\chi$ is exceptional. Assume that $\chi$ is ordinary. We first show that $W\left(h_{l}\right)=0$ if $-\mu \leqslant l<0$. Take a $y \in \mathcal{O}_{F}$ such that $\operatorname{ord}_{F} y=\mu+\delta-1$ and $\chi(z) \neq 1$ with $z=1+\theta y$. Then $\iota\left(z^{\sigma} / z\right) h_{l}=h_{l} u$, where

$$
u=\frac{1}{z}\left(\begin{array}{cc}
1 & \pi^{l} m \mathrm{~N}(\alpha \theta) y \\
-y / \pi^{l} m \mathrm{~N}(\alpha) & 1+\operatorname{Tr}(\theta) y
\end{array}\right) .
$$

If $-\mu \leqslant l<0$, we have $u \in \mathcal{U}^{\prime}$ and, hence, $\left(\chi / \chi_{0}\right)(z) W\left(h_{l}\right)=\chi_{0}^{-1}(z) W\left(h_{l}\right)$. This implies that $W\left(h_{l}\right)=0$. A similar argument shows that $W\left(h_{l} \mathbf{n}(a) w_{0}\right)=0$ if $-\mu \leqslant l \leqslant 0$ and $a \in \mathfrak{p}_{F}$.

To prove Theorem 10.1, we first observe that $\Phi_{0}\left(\alpha h^{-1} \eta\right)=0$ if $h=h_{l}(l \geqslant 1)$ or $h=$ $h_{l} \mathbf{n}(a) w_{0}\left(l \geqslant 1, a \in \pi \mathcal{O}_{F}\right)$. In view of Lemmas 11.4 and 11.5 , we obtain $\mathcal{I}_{\mathfrak{a}}(W, \tau)=I_{1}+\delta_{e}(\chi) I_{2}$, where

$$
\begin{aligned}
& I_{1}=|\mathrm{N}(\alpha)|_{F} \chi_{0}\left(\alpha^{\sigma} / \alpha\right) \frac{2}{1+p} p^{\mu+1} W\left(h_{0}\right) \int_{K} \mathcal{M}_{\chi_{0}}^{T}\left(h_{0}\right) \varphi_{0}(-w) \overline{\tau\left(w, x_{w}\right)} d w, \\
& I_{2}=|\mathrm{N}(\alpha)|_{F} \chi_{0}\left(\alpha^{\sigma} / \alpha\right) \frac{2}{1+p} W\left(h_{0} w_{0}\right) \int_{K} \mathcal{M}_{\chi_{0}}^{T}\left(h_{0} w_{0}\right) \varphi_{0}(-w) \overline{\tau\left(w, x_{w}\right)} d w .
\end{aligned}
$$

Since $\mathcal{M}_{\chi_{0}}^{T}\left(h_{0}\right) \varphi_{0}(-w)=\chi_{0}(\alpha)|\mathrm{N}(\alpha)|_{F}^{-1 / 2} \varphi_{0}\left(\alpha^{-1} w\right)$ and $\operatorname{vol}(\mathfrak{a})=|\mathrm{N}(\alpha)|_{F} p^{-\delta / 2}$, we have

$$
\begin{aligned}
I_{1} & =\frac{2}{1+p} p^{\mu+1} \chi_{0}\left(\alpha^{\sigma} / \alpha\right) W\left(h_{0}\right) \chi_{0}(\alpha)|\mathrm{N}(\alpha)|_{F}^{1 / 2} \int_{\mathfrak{a}} \overline{\tau\left(w, x_{w}\right)} d w \\
& =\frac{2}{1+p} p^{\mu+1-\delta / 2} \chi_{0}^{-1}(\alpha)|\mathrm{N}(\alpha)|_{F}^{3 / 2} W\left(h_{0}\right) \overline{\tau(1)} .
\end{aligned}
$$

We next have $\mathcal{M}_{\chi_{0}}^{T}\left(h_{0} w_{0}\right) \varphi_{0}(-w)=\chi_{0}(\alpha)|\mathrm{N}(\alpha)|_{F}^{-1 / 2} p^{-\delta / 2} \lambda_{K}(\psi) \varphi_{0}\left(\Pi^{\delta} \alpha^{-1} w\right)$ and, hence,

$$
I_{2}=\frac{2}{1+p} \chi_{0}^{-1}(\alpha)|\mathrm{N}(\alpha)|_{F}^{1 / 2} p^{-\delta / 2} \lambda_{K}(\psi) W\left(h_{0} w_{0}\right) \int_{\Pi^{-\delta} \mathfrak{a}} \overline{\tau\left(w, x_{w}\right)} d w .
$$

To complete the proof of Theorem 10.1, it now remains to show the following.

Lemma 11.6. Suppose that $\chi \in \mathcal{X}_{\text {prim }}^{+}(m, \mathfrak{a})$ is exceptional. For $\tau \in \mathcal{T}^{m}(\mathfrak{a}, \chi)$, we have

$$
\int_{\Pi^{-\delta} \mathfrak{a}} \tau\left(w, x_{w}\right) d w=\lambda_{K}\left(\psi_{m}\right) \chi(\theta)|\mathrm{N}(\alpha)|_{F} p^{1 / 2} \tau(1) .
$$

Proof. Put $t_{\theta}=\theta^{\sigma} / \theta \in K^{1}$. By (10.5), taking a sufficiently large lattice $L^{\prime}$ of $K$, we have

$$
\begin{aligned}
\tau\left(r t_{\theta}\right) & =\lambda_{K}\left(\psi_{m}\right)^{-1} \chi\left(\frac{1-t_{\theta}}{\kappa}\right)\left|\mathrm{N}\left(1-t_{\theta}\right)\right|_{F}^{1 / 2} \int_{L^{\prime}} \psi_{m}\left(\frac{1}{2}\left\langle w, t_{\theta} w\right\rangle_{\kappa}\right) \tau\left(r\left(w\left(1-t_{\theta}\right), 0\right)\right) d^{m} w \\
& =\lambda_{K}\left(\psi_{m}\right)^{-1} \chi^{-1}(\theta)|\mathrm{N}(m \theta)|_{F}^{1 / 2} \int_{L} \psi\left(\frac{m}{2} \operatorname{Tr}\left(\frac{\kappa}{1-t_{\theta}}\right) w w^{\sigma}\right) \tau(r(w, 0)) d w \\
& =\lambda_{K}\left(\psi_{m}\right)^{-1} \chi^{-1}(\theta)|m|_{F} p^{-1 / 2} \int_{L} \tau\left(r\left(w, x_{w}\right)\right) d w
\end{aligned}
$$

where $L=\left(1-t_{\theta}\right)^{-1} L^{\prime}$. We may (and do) suppose that $L \supset \Pi^{-\delta} \mathfrak{a}$. Since $\chi$ is exceptional, the last integral is equal to

We thus have

$$
\begin{aligned}
& \int_{L} \int_{\Pi^{-1} \mathfrak{a}} \tau\left(r\left(w, x_{w}\right)\left(w^{\prime}, x_{w^{\prime}}\right)\right) d_{\Pi^{-1} \mathfrak{a}} w^{\prime} d w \\
& \quad=\int_{L} \tau\left(r\left(w, x_{w}\right)\right) \int_{\Pi^{-1} \mathfrak{a}} \psi_{m}\left(-\operatorname{Tr}\left(\theta^{\sigma} w^{\sigma} w^{\prime}\right)\right) d_{\Pi^{-1} \mathfrak{a}} w^{\prime} d w=\int_{\Pi^{-\delta} \mathfrak{a}} \tau\left(r\left(w, x_{w}\right)\right) d w .
\end{aligned}
$$

$$
\int_{\Pi^{-\delta} \mathfrak{a}} \tau\left(w, x_{w}\right) d w=\lambda_{K}\left(\psi_{m}\right) \chi(\theta)|m|_{F}^{-1} p^{1 / 2} \tau\left(t_{\theta}\right)=\lambda_{K}\left(\psi_{m}\right) \chi(\theta)|\mathrm{N}(\alpha)|_{F} p^{1 / 2} \tau(1),
$$

which proves the lemma. 


\section{A. Murase And T. Sugano}

Remark. When $\delta=1$, the left-hand side of (11.2) is independent of the choice of $\theta$ and equal to $|\mathrm{N}(\alpha)|_{F} p^{1 / 2} \tau(1)$ by the assumption that $\chi$ is exceptional. This implies that $\lambda_{K}\left(\psi_{m}\right) \chi(\theta)=1$ (we can show this fact directly). Note that, if $\delta \geqslant 2$, the left-hand side of (11.2) depends on the choice of $\theta$.

\subsection{Split case}

In this subsection, we consider the case where $K=F \oplus F$, and let $\Pi_{1}=(\pi, 1), \Pi_{2}=(1, \pi)$. We may (and do) take $\theta=(1,0)$. Then $x_{w}=\frac{1}{2} \mathrm{~N}(w)$ for $w \in K$. Let $Z=\left\{t 1_{2} \mid t \in K^{1}\right\}$ be the center of $H$. For $l \in \mathbb{Z}$, we put $h_{l}=\mathbf{d}\left(\Pi_{1}^{-l} \alpha^{-1}\right)$.

Lemma 11.7. We have $H=\bigcup_{l \geqslant-\mu} Z H_{\eta} h_{l} \mathcal{U}$.

Proof. For $h, h^{\prime} \in H$, we write $h \sim h^{\prime}$ if $h^{\prime} \in Z H_{\eta} h \mathcal{U}$. We first show that, for any $h \in H$, there exists an $l \in \mathbb{Z}$ such that $h \sim h_{l}$. By Iwasawa decomposition of $H$, we have $h=\overline{\mathbf{n}}(x) \mathbf{d}(a) u$ for some $x \in F, a \in K^{\times}, u \in \mathcal{U}$. We may (and do) assume that $x \neq m^{-1}$. Putting $y=(1-m x)^{-1} \in F^{\times}$, we have

$$
\overline{\mathbf{n}}(x)=\iota\left(\left(y, y^{-1}\right)\right) \mathbf{d}\left(\left(1, y^{-1}\right)\right)
$$

and, hence, $h \sim \mathbf{d}\left(a^{\prime}\right)$ for some $a^{\prime} \in K^{\times}$. Our claim immediately follows from this. It is now sufficient to show that $h_{l} \sim h_{-\mu}$ if $l<-\mu$. Put $y^{\prime}=\left(1-\pi^{l} m \mathrm{~N}(\alpha)\right)^{-1} \in F^{\times}$. Note that $\operatorname{ord}_{F} y^{\prime}=-(l+\mu)$. By (11.3), we have $h_{l} \sim h_{l} \overline{\mathbf{n}}(1)=\overline{\mathbf{n}}\left(\pi^{l} \mathrm{~N}(\alpha)\right) h_{l} \sim \mathbf{d}\left(\left(1,1 / y^{\prime}\right)\right) h_{l} \sim \mathbf{d}\left(\left(1 / y^{\prime}, 1\right)\right) h_{l} \sim \mathbf{d}\left(\Pi_{1}^{l+\mu}\right) h_{l}=h_{-\mu}$.

Lemma 11.8. For $l \geqslant-\mu$, we have

$$
v_{l}:=\operatorname{vol}\left(Z H_{\eta} \backslash Z H_{\eta} h_{l} \mathcal{U}\right)= \begin{cases}1 & \text { if } l=-\mu, \\ p^{l+\mu}\left(1-p^{-1}\right) & \text { if } l>-\mu .\end{cases}
$$

Proof. Let $l \geqslant-\mu$ and put $X=Z_{0} H_{\eta, 0} h_{l} \mathcal{U}$, where $Z_{0}=\left\{t 1_{2} \mid t \in \mathcal{O}_{K}^{1}\right\}$ and $H_{\eta, 0}=\left\{\iota(t) \mid t \in \mathcal{O}_{K}^{1}\right\}$. Then $v_{l}=\operatorname{vol}(X)$. Since $\iota(t) \eta^{\sigma}=t \cdot \eta^{\sigma}\left(t \in K^{1}\right)$, we have

$$
X=\left\{h \in H \mid \Pi_{1}^{l} \alpha \cdot h^{-1} \eta, \Pi_{1}^{l} \alpha \cdot h^{-1} \eta^{\sigma} \in \mathcal{O}_{K}^{2}-\left(\Pi_{1} \mathcal{O}_{K}^{2} \cup \Pi_{2} \mathcal{O}_{K}^{2}\right)\right\} .
$$

It follows that $X=Y \cup \bigcup_{k=1}^{l+\mu} Y_{k}$ (disjoint union), where

$$
\begin{gathered}
Y=\left\{\mathbf{n}(x) \mathbf{d}(y) u \mid x \in \pi^{-l} \mathrm{~N}(\alpha)^{-1} \mathcal{O}_{F}, y \in \Pi_{1}^{-l} \alpha^{-1} \mathcal{O}_{K}^{\times}, u \in \mathcal{U}\right\}, \\
Y_{k}=\left\{\mathbf{n}(x) \mathbf{d}(y) u \mid x,-x+m \in \pi^{k-l} \mathrm{~N}(\alpha)^{-1} \mathcal{O}_{F}^{\times}, y \in \Pi_{1}^{k-l} \Pi_{2}^{k} \alpha^{-1} \mathcal{O}_{K}^{\times}, u \in \mathcal{U}\right\} .
\end{gathered}
$$

The lemma now follows from

$$
\operatorname{vol}(Y)=1, \quad \operatorname{vol}\left(Y_{k}\right)=p^{k} \times \begin{cases}1-p^{-1} & \text { if } 1 \leqslant k \leqslant l+\mu-1, \\ 1-2 p^{-1} & \text { if } k=l+\mu\end{cases}
$$

Lemma 11.9. For $W \in \mathcal{W}^{m}(\chi)$, we have $\operatorname{Supp}(W) \subset \bigcup_{l \geqslant 0} Z H_{\eta} h_{l} \mathcal{U}$.

Proof. This is proved by an argument similar to the proof of Lemma 11.2.

By Lemma 11.9, we have

$$
\begin{aligned}
\mathcal{I}_{\mathfrak{a}}(W, \tau)= & |\mathrm{N}(\alpha)|_{F} \sum_{k \in \mathbb{Z}} \sum_{l=0}^{\infty} v_{l} \chi_{0}^{-1}\left(\operatorname{det} \mathbf{d}\left(\Pi_{1}^{-k-l} \Pi_{2}^{k} \alpha^{-1}\right)\right) \Phi_{0}\left(\alpha \mathbf{d}\left(\Pi_{1}^{k+l} \Pi_{2}^{-k} \alpha\right) \eta\right) \\
& \times W\left(\mathbf{d}\left(\Pi_{1}^{-k-l} \Pi_{2}^{k} \alpha^{-1}\right)\right) \int_{K}\left(\mathcal{M}_{\chi_{0}}^{T}\left(\mathbf{d}\left(\Pi_{1}^{-k-l} \Pi_{2}^{k} \alpha^{-1}\right)\right) \varphi_{0}\right)(-w) \overline{\tau\left(w, x_{w}\right)} d w .
\end{aligned}
$$




\section{ON THE Fourier-JACOBI EXPANSION OF THE UNITARY KUDLA LIFT}

For $k \in \mathbb{Z}$ and $l \geqslant 0$, we have $\Phi_{0}\left(\alpha \mathbf{d}\left(\Pi_{1}^{k+l} \Pi_{2}^{-k} \alpha\right) \eta\right)=\delta(k=l=0)$. By this and Lemma 11.8, we obtain

$$
\begin{aligned}
\mathcal{I}_{\mathfrak{a}}(W, \tau) & =\chi_{0}^{-1}(\alpha)|\mathrm{N}(\alpha)|_{F}^{1 / 2} v_{0} \cdot W\left(\mathbf{d}\left(\alpha^{-1}\right)\right) \int_{K} \varphi_{0}\left(\alpha^{-1} w\right) \overline{\tau\left(w, x_{w}\right)} d w \\
& =\chi_{0}^{-1}(\alpha)|\mathrm{N}(\alpha)|_{F}^{3 / 2} \overline{\tau(1)} W\left(\mathbf{d}\left(\alpha^{-1}\right)\right) \times p^{\mu} \begin{cases}1-p^{-1} & \text { if } \mu>0, \\
1 & \text { if } \mu=0 .\end{cases}
\end{aligned}
$$

This completes the proof of Theorem 10.1 in the split case.

\section{Calculation of local integrals at finite primes: non-primitive case}

12.1 In this section, we let $\Omega \in X_{\text {unr }}\left(K^{1}\right)$ and $W \in \mathcal{W}^{m}\left(\chi ; \chi_{0} \Omega\right)$ (cf. $\S 10.1$ ), and prove Theorem 10.2. We keep the notation of $\S 11$.

\subsection{Inert case}

In this subsection, we consider the case where $K / F$ is inert. For $k \in \mathbb{Z}$, we have

$$
\mathcal{I}_{\pi^{k} \mathfrak{a}}(W, \tau)=\left|\mathrm{N}\left(\pi^{k} \alpha\right)\right|_{F} \int_{H_{\eta} \backslash H} \int_{K} \chi_{0}^{-1}(\operatorname{det} h) W(h) A_{k}(w, h) \overline{\tau\left(w, x_{w}\right)} d w d h,
$$

where $A_{k}(w, h)=\Phi_{0}\left(\pi^{k} \alpha h^{-1} \eta\right)\left(\mathcal{M}_{\chi_{0}}^{T}(h) \varphi_{0}\right)(-w)$. In view of Lemma 10.3 and the fact that $\phi_{1}^{\vee}=\phi_{1}$, we have

$$
\mathcal{I}_{\pi^{k} \mathfrak{a}}\left(W * \phi_{1}, \tau\right)=\left|\mathrm{N}\left(\pi^{k} \alpha\right)\right|_{F} \int_{H_{\eta} \backslash H} \int_{K} \chi_{0}^{-1}(\operatorname{det} h) W(h) A_{k}^{\prime}(w, h) \overline{\tau\left(w, x_{w}\right)} d w d h,
$$

where

$$
\begin{aligned}
A_{k}^{\prime}(w, h)= & -\Phi_{0}\left(\pi^{k} \alpha \mathbf{d}(\pi) h^{-1} \eta\right)\left(\mathcal{M}_{\chi 0}^{T}\left(h \mathbf{d}\left(\pi^{-1}\right)\right) \varphi_{0}\right)(-w) \\
& -\sum_{a \in\left(\mathcal{O}_{F}-\pi \mathcal{O}_{F}\right) / \pi \mathcal{O}_{F}} \Phi_{0}\left(\pi^{k} \alpha \mathbf{n}\left(-\pi^{-1} a\right) h^{-1} \eta\right)\left(\mathcal{M}_{\chi_{0}}^{T}\left(h \mathbf{n}\left(\pi^{-1} a\right)\right) \varphi_{0}\right)(-w) \\
& -\sum_{a \in \mathcal{O}_{F} / \pi^{2} \mathcal{O}_{F}} \Phi_{0}\left(\pi^{k} \alpha \mathbf{d}\left(\pi^{-1}\right) \mathbf{n}(-a) h^{-1} \eta\right)\left(\mathcal{M}_{\chi_{0}}^{T}(h \mathbf{n}(a) \mathbf{d}(\pi)) \varphi_{0}\right)(-w) .
\end{aligned}
$$

It follows that

$$
\begin{aligned}
& p^{2} \mathcal{I}_{\pi^{k+1} \mathfrak{a}}(W, \tau)-p^{-1} \mathcal{I}_{\pi^{k} \mathfrak{a}}\left(W * \phi_{1}, \tau\right)+\left\{\delta(k+\mu>0) p^{-1}-1\right\} \mathcal{I}_{\pi^{k} \mathfrak{a}}(W, \tau)+p^{-2} \mathcal{I}_{\pi^{k-1} \mathfrak{a}}(W, \tau) \\
& \quad=\left|\mathrm{N}\left(\pi^{k} \alpha\right)\right|_{F} \int_{H_{\eta} \backslash H} \int_{K} \chi_{0}^{-1}(\operatorname{det} h) W(h) B_{k}(w, h) \overline{\tau\left(w, x_{w}\right)} d w d h,
\end{aligned}
$$

where

$$
B_{k}(w, h)=A_{k+1}(w, h)-p^{-1} A_{k}^{\prime}(w, h)+\left\{\delta(k+\mu>0) p^{-1}-1\right\} A_{k}(w, h)+A_{k-1}(w, h) .
$$

Set

$$
\mathcal{B}_{k, l}=\int_{K} B_{k}\left(w, h_{l}\right) \overline{\tau\left(w, x_{w}\right)} d w .
$$

To prove Theorem 10.2, it suffices to show the following result in view of Lemma 11.2.

Proposition 12.1. For $k, l \geqslant 0$, we have $\mathcal{B}_{k, l}=0$.

Proof. For $l \in \mathbb{Z}$, set $\phi_{l}(w)=\varphi_{0}\left(\pi^{-l} \alpha^{-1} w\right)$. Let $l \geqslant 0$ and $k \in \mathbb{Z}$. Observe that

$$
A_{k}\left(w, h_{l}\right)=\delta(k \geqslant l)(-p)^{l} \chi_{0}(\alpha)|\mathrm{N}(\alpha)|_{F}^{-1 / 2} \phi_{l}(w)
$$




\section{A. Murase And T. Sugano}

and that

$$
A_{k}^{\prime}\left(w, h_{l}\right)=(-1)^{l} p^{l+1} \chi_{0}(\alpha)|\mathrm{N}(\alpha)|_{F}^{-1 / 2}\left\{\delta(k \geqslant l+1) \phi_{l+1}(w)-p^{-1} \delta(k \geqslant l+1) I_{1}(w)+p^{-2} I_{2}(w)\right\},
$$

where

$$
\begin{gathered}
I_{1}(w)=\sum_{a \in\left(\mathcal{O}_{F}-\pi \mathcal{O}_{F}\right) / \pi \mathcal{O}_{F}} \psi\left(-\pi^{-2 l-1} \mathrm{~N}(\alpha)^{-1} \mathrm{~N}(w) a\right) \phi_{l}(w), \\
I_{2}(w)=\sum_{a \in \mathcal{O}_{F} / \pi^{2} \mathcal{O}_{F}} \Phi_{0}\left(\begin{array}{c}
\pi^{k+l-1} m \mathrm{~N}(\alpha) \theta^{\sigma}-\pi^{k-l-1} a \\
\pi^{k+1-l}
\end{array}\right) \psi\left(-\pi^{-2 l} \mathrm{~N}(\alpha)^{-1} \mathrm{~N}(w) a\right) \phi_{l-1}(w) .
\end{gathered}
$$

A straightforward calculation shows that

$$
\begin{gathered}
I_{1}(w)=p \phi_{l+1}(w)-\phi_{l}(w), \\
I_{2}(w)=\delta(k+l+\mu>0)\left[\delta(k \geqslant l+1) p^{2} \phi_{l}(w)+\delta(k=l) p \phi_{l}(w)+\delta(k=l-1) \phi_{l-1}(w)\right]
\end{gathered}
$$

and, hence, that

$$
\begin{aligned}
A_{k}^{\prime}\left(w, h_{l}\right)= & (-1)^{l} p^{l+1} \chi_{0}(\alpha)|\mathrm{N}(\alpha)|_{F}^{-1 / 2} \times \delta(k+l+\mu>0)\left[\delta(k=l) p^{-1} \phi_{l}(w)\right. \\
& \left.+\delta(k \geqslant l+1)\left(1+p^{-1}\right) \phi_{l}(w)+\delta(k=l-1) p^{-2} \phi_{l-1}(w)\right] .
\end{aligned}
$$

This implies that, for $k, l \geqslant 0$,

$$
B_{k}\left(w, h_{l}\right)=(-p)^{l} \chi_{0}(\alpha)|\mathrm{N}(\alpha)|_{F}^{-1 / 2} \delta(l=k+1)\left\{\phi_{l}(w)-p^{-2} \phi_{l-1}(w)\right\}
$$

and, hence,

$$
\mathcal{B}_{k, l}=(-p)^{l} \chi_{0}(\alpha)|\mathrm{N}(\alpha)|_{F}^{-1 / 2} \delta(k=l-1)\left(J_{l}-p^{-2} J_{l-1}\right),
$$

where

$$
J_{l}=\int_{K} \phi_{l}(w) \overline{\tau\left(w, x_{w}\right)} d w .
$$

Since $J_{l}=p^{-2 l}|\mathrm{~N}(\alpha)|_{F} \overline{\tau(1)}$ for $l \geqslant 0$, we have proved that $\mathcal{B}_{k, l}=0$.

\subsection{Ramified case}

In this subsection, we consider the case where $K / F$ is a ramified quadratic extension. For $k \in \mathbb{Z}$, we have

$$
\mathcal{I}_{\Pi^{k} \mathfrak{a}}(W, \tau)=p^{-k}|\mathrm{~N}(\alpha)|_{F} \int_{H_{\eta} \backslash H} \int_{K} \chi_{0}^{-1}(\operatorname{det} h) A_{k}(w, h) W(h) \overline{\tau\left(w, x_{w}\right)} d w d h,
$$

where $A_{k}(w, h)=\Phi_{0}\left(\Pi^{k} \alpha h^{-1} \eta\right)\left(\mathcal{M}_{\chi_{0}}^{T}(h) \varphi_{0}\right)(-w)(w \in K, h \in H)$. By Lemma 10.3 and the fact that $\left(\phi^{ \pm}\right)^{\vee}=\phi^{\mp}$, we obtain

$$
\mathcal{I}_{\Pi^{k} \mathfrak{a}}\left(W *\left(\phi^{+}+\phi^{-}\right), \tau\right)=p^{-k}|\mathrm{~N}(\alpha)|_{F} \int_{H_{\eta} \backslash H} \int_{K} \chi_{0}^{-1}(\operatorname{det} h) A_{k}^{\prime}(w, h) W(h) \overline{\tau\left(w, x_{w}\right)} d w d h,
$$

where

$$
A_{k}^{\prime}(w, h)=\chi_{0}(\Pi) \sum_{c \in \mathcal{O}_{F} / \pi \mathcal{O}_{F}} A_{k}(w, h \mathbf{n}(c) \mathbf{d}(\Pi))+\chi_{0}^{-1}(\Pi) \sum_{c \in \mathcal{O}_{F} / \pi \mathcal{O}_{F}} A_{k}\left(w, h \overline{\mathbf{n}}(D c) \mathbf{d}\left(\Pi^{-1}\right)\right) .
$$

It follows that

$$
\begin{aligned}
& p \mathcal{I}_{\Pi^{k+1} \mathfrak{a}}(W, \tau)-p^{-1 / 2} \mathcal{I}_{\Pi^{k} \mathfrak{a}}\left(W *\left(\phi^{+}+\phi^{-}\right), \tau\right) \\
& \quad-\delta(k=0 \text { and } \chi \text { is ordinary }) \mathcal{I}_{\Pi^{k} \mathfrak{a}}(W, \tau)+p^{-1} \mathcal{I}_{\Pi^{k-1} \mathfrak{a}}(W, \tau) \\
& =p^{-k}|\mathrm{~N}(\alpha)| \int_{H_{\eta} \backslash H} d h \int_{K} d w \chi_{0}^{-1}(\operatorname{det} h) W(h) B_{k}(w, h) \overline{\tau\left(w, x_{w}\right)},
\end{aligned}
$$




\section{ON THE Fourier-JACOBI EXPANSION OF THE UNITARY KUDLA LIFT}

where

$$
B_{k}(w, h)=A_{k+1}(w, h)-p^{-1 / 2} A_{k}^{\prime}(w, h)-\delta(k=0 \text { and } \chi \text { is ordinary }) A_{k}(w, h)+A_{k-1}(w, h) .
$$

Set $\phi_{l}(w)=\varphi_{0}\left(\Pi^{-l} \alpha^{-1} w\right)$ for $l \in \mathbb{Z}$.

LEMMA 12.2. Let $k \geqslant 0$.

(i) For $l \geqslant 0$, we have

$$
\begin{aligned}
B_{k}\left(w, h_{l}\right)= & \chi_{0}\left(\Pi^{l} \alpha\right) p^{l / 2}|\mathrm{~N}(\alpha)|_{F}^{-1 / 2}\left[\delta(k=l \text { or } l-1)\left\{\phi_{l}(w)-p^{-1} \phi_{l-1}(w)\right\}\right. \\
& \left.-\delta(l=k=0 \text { and } \chi \text { is ordinary }) \phi_{0}(w)\right] .
\end{aligned}
$$

(ii) For $l \geqslant 0$ and $a \in \mathfrak{p}_{F}$, we have

$$
B_{k}\left(w, h_{l} \mathbf{n}(a) w_{0}\right)= \begin{cases}-\beta_{l} \cdot \psi\left(a \mathrm{~N}\left(\Pi^{-l} \alpha^{-1} w\right)\right) \phi_{l-\delta}(w) & \text { if } k=l=0 \text { and } \chi \text { is ordinary } \\ 0 & \text { otherwise, }\end{cases}
$$

where $\beta_{l}=p^{(l-\delta) / 2}|\mathrm{~N}(\alpha)|_{F}^{-1 / 2} \chi_{0}\left(\Pi^{l} \alpha\right) \lambda_{K}(\psi)$.

Proof. First observe that $A_{k}\left(w, h_{l}\right)=\delta(l \leqslant k) \chi_{0}\left(\Pi^{l} \alpha\right) p^{l / 2}|\mathrm{~N}(\alpha)|_{F}^{-1 / 2} \phi_{l}(w)$. Using the formulas

$$
\sum_{c \in \mathcal{O}_{F} / \pi \mathcal{O}_{F}} \mathcal{M}_{\chi_{0}}^{T}\left(\mathbf{n}\left(\pi^{-1} c\right)\right) \varphi_{0}(w)=p \varphi_{0}\left(\Pi^{-1} w\right)
$$

and

$$
\sum_{c \in \mathcal{O}_{F} / \pi \mathcal{O}_{F}} \mathcal{M}_{\chi_{0}}^{T}\left(\overline{\mathbf{n}}\left(\pi^{-1} D c\right)\right) \varphi_{0}(w)=\varphi_{0}(\Pi w)
$$

we obtain

$$
\begin{aligned}
A_{k}^{\prime}\left(w, h_{l}\right)= & \chi_{0}(\Pi) \sum_{c \in \mathcal{O}_{F} / \pi \mathcal{O}_{F}} \Phi_{0}\left(\begin{array}{c}
\Pi^{k+(l-1) \sigma} m \mathrm{~N}(\alpha) \theta^{\sigma}-\Pi^{k-l-\sigma} c \\
\Pi^{k+1-l}
\end{array}\right) \mathcal{M}_{\chi_{0}}^{T}\left(h_{l-1} \mathbf{n}\left(\pi^{-1} c\right)\right) \varphi_{0}(-w) \\
& +\chi_{0}^{-1}(\Pi) \sum_{c \in \mathcal{O}_{F} / \pi \mathcal{O}_{F}} \Phi_{0}\left(\begin{array}{c}
\Pi^{k+(l+1) \sigma} m \mathrm{~N}(\alpha) \theta^{\sigma} \\
-\Pi^{k-1+l \sigma} m \mathrm{~N}(\alpha) D c \theta^{\sigma}+\Pi^{k-l-1}
\end{array}\right) \mathcal{M}_{\chi_{0}}^{T}\left(h_{l+1} \overline{\mathbf{n}}\left(\pi^{-1} D c\right)\right) \varphi_{0}(-w) \\
= & \chi_{0}\left(\Pi^{l} \alpha\right)\left\{\delta(k \geqslant l-1)\left|\mathrm{N}\left(\Pi^{-l+1} \alpha^{-1}\right)\right|_{F}^{1 / 2} \phi_{l-1}(w)\right. \\
& +\delta(k \geqslant l+1)\left|\mathrm{N}\left(\Pi^{-l+1} \alpha^{-1}\right)\right|_{F}^{1 / 2} \sum_{c \in \mathcal{O}_{F}^{\times} / \pi \mathcal{O}_{F}} \mathcal{M}_{\chi_{0}}^{T}\left(\mathbf{n}\left(\pi^{-1} c\right)\right) \varphi_{0}\left(-\Pi^{-l+1} \alpha^{-1} w\right) \\
& \left.+\delta(k \geqslant l+1)\left|\mathrm{N}\left(\Pi^{-l-1} \alpha^{-1}\right)\right|_{F}^{1 / 2} \sum_{c \in \mathcal{O}_{F} / \pi \mathcal{O}_{F}} \mathcal{M}_{\chi_{0}}^{T}\left(\overline{\mathbf{n}}\left(\pi^{-1} D c\right)\right) \varphi_{0}\left(-\Pi^{-l-1} \alpha^{-1} w\right)\right\} \\
= & \chi_{0}\left(\Pi^{l} \alpha\right) p^{(l+1) / 2}|\mathrm{~N}(\alpha)|_{F}^{-1 / 2}\left\{\delta(k=l \text { or } l-1) p^{-1} \phi_{l-1}(w)+2 \cdot \delta(k \geqslant l+1) \phi_{l}(w)\right\} .
\end{aligned}
$$

The assertion (i) is now proved by a straightforward calculation. Next let $h=h_{l} \mathbf{n}(a) w_{0}(l \geqslant 0$, $\left.a \in \mathfrak{p}_{F}\right)$. A similar argument as above shows that $A_{k}(w, h)=\delta(k \geqslant l) \beta_{l} \cdot \psi\left(a \mathrm{~N}\left(\Pi^{-l} \alpha^{-1} w\right)\right) \phi_{l-\delta}(w)$ and $A_{k}^{\prime}(w, h)=\{\delta(k \geqslant l+1)+\delta(k \geqslant l-1)\} p^{1 / 2} \beta_{l} \cdot \psi\left(a \mathrm{~N}\left(\Pi^{-l} \alpha^{-1} w\right)\right) \phi_{l-\delta}(w)$. The second assertion immediately follows from these.

Proposition 12.3. Let $\tau \in \mathcal{T}^{m}(\mathfrak{a}, \chi)$.

(i) For $k, l \geqslant 0$, we have

$$
\int_{K} B_{k}\left(w, h_{l}\right) \overline{\tau\left(w, x_{w}\right)} d w=0
$$




\section{A. Murase and T. Sugano}

(ii) For $k \geqslant 0, l \geqslant 1$ and $a \in \mathfrak{p}_{F}$, we have

$$
\int_{K} B_{k}\left(w, h_{l} \mathbf{n}(a) w_{0}\right) \overline{\tau\left(w, x_{w}\right)} d w=0 .
$$

(iii) Assume that $\chi$ is exceptional. Then, for $k \geqslant 0$, we have

$$
\int_{K} B_{k}\left(w, h_{0} w_{0}\right) \overline{\tau\left(w, x_{w}\right)} d w=0 .
$$

Proof. For $l \in \mathbb{Z}$, set

$$
J_{l}=\int_{K} \phi_{l}(w) \overline{\tau\left(w, x_{w}\right)} d w .
$$

In view of (10.2) and (10.4), we have

$$
J_{l}= \begin{cases}p^{-l-\delta / 2}|\mathrm{~N}(\alpha)|_{F} \overline{\tau(1)} & \text { if } l \geqslant 0 \text { or ' } \mu=0, \chi \text { is exceptional and } l=-1 \\ 0 & \text { otherwise }\end{cases}
$$

for $l \geqslant-1$. By Lemma 12.2, for $k, l \geqslant 0$, we have

$$
\begin{aligned}
& \int_{K} B_{k}\left(w, h_{l}\right) \overline{\tau\left(w, x_{w}\right)} d w \\
& \quad=\chi_{0}\left(\Pi^{l} \alpha\right) p^{l / 2}|\mathrm{~N}(\alpha)|_{F}^{-1 / 2}\left\{\delta(k=l \text { or } l-1)\left(J_{l}-p^{-1} J_{l-1}\right)-\delta(l=k=0 \text { and } \chi \text { is ordinary }) J_{0}\right\} \\
& \quad=0,
\end{aligned}
$$

which shows assertion (i). The assertions (ii) and (iii) directly follow from Lemma 12.2(ii).

In view of Lemma 11.5 and Proposition 12.3, we have completed the proof of Theorem 10.2 in the ramified case.

\subsection{Split case}

In this subsection, we consider the case where $K=F \oplus F$ and use the notation of $\S 11.4$.

LEMMA 12.4. Let $k_{1}, k_{2} \in \mathbb{Z}$.

(i) We have

$$
\mathcal{I}_{\Pi_{1}^{k_{1}} \Pi_{2}^{k_{2}} \mathfrak{a}}(W, \tau)=p^{-k_{1}-k_{2}}|\mathrm{~N}(\alpha)|_{F} \int_{H_{\eta} \backslash H} \int_{K} \chi_{0}^{-1}(\operatorname{det} h) W(h) A_{k_{1}, k_{2}}(w, h) \overline{\tau\left(w, x_{w}\right)} d w d h,
$$

where $A_{k_{1}, k_{2}}(w, h)=\Phi_{0}\left(\Pi_{1}^{k_{1}} \Pi_{2}^{k_{2}} \alpha h^{-1} \eta\right)\left(\mathcal{M}_{\chi_{0}}^{T}(h) \varphi_{0}\right)(-w)$.

(ii) We have

$$
\mathcal{I}_{\Pi_{1}^{k_{1}} \Pi_{2}^{k_{2}} \mathfrak{a}}\left(W * \phi_{1}, \tau\right)=p^{-k_{1}-k_{2}}|\mathrm{~N}(\alpha)|_{F} \int_{H_{\eta} \backslash H} \int_{K} \chi_{0}^{-1}(\operatorname{det} h) W(h) A_{k_{1}, k_{2}}^{1}(w, h) \overline{\tau\left(w, x_{w}\right)} d w d h
$$

where

$$
\begin{aligned}
A_{k_{1}, k_{2}}^{1}(w, h)= & \chi_{0}^{-1}\left(\Pi_{2}\right)\left\{\Phi_{0}\left(\Pi_{1}^{k_{1}} \Pi_{2}^{k_{2}} \alpha \mathbf{d}\left(\Pi_{2}\right) h^{-1} \eta\right)\left(\mathcal{M}_{\chi_{0}}^{T}\left(h \mathbf{d}\left(\Pi_{2}^{-1}\right)\right) \varphi_{0}\right)(-w)\right. \\
& \left.+\sum_{c \in \mathcal{O}_{F} / \pi \mathcal{O}_{F}} \Phi_{0}\left(\Pi_{1}^{k_{1}} \Pi_{2}^{k_{2}} \alpha \mathbf{d}\left(\Pi_{1}^{-1}\right) \mathbf{n}(-c) h^{-1} \eta\right)\left(\mathcal{M}_{\chi_{0}}^{T}\left(h \mathbf{n}(c) \mathbf{d}\left(\Pi_{1}\right)\right) \varphi_{0}\right)(-w)\right\} .
\end{aligned}
$$

(iii) We have

$$
\mathcal{I}_{\Pi_{1}^{k_{1}} \Pi_{2}^{k_{2} \mathfrak{a}}}\left(W * \phi_{2}, \tau\right)=p^{-k_{1}-k_{2}}|\mathrm{~N}(\alpha)|_{F} \int_{H_{\eta} \backslash H} \int_{K} \chi_{0}^{-1}(\operatorname{det} h) W(h) A_{k_{1}, k_{2}}^{2}(w, h) \overline{\tau\left(w, x_{w}\right)} d w d h,
$$


where

$$
\begin{aligned}
A_{k_{1}, k_{2}}^{2}(w, h)= & \chi_{0}^{-1}\left(\Pi_{1}\right)\left\{\Phi_{0}\left(\Pi_{1}^{k_{1}} \Pi_{2}^{k_{2}} \alpha \mathbf{d}\left(\Pi_{1}\right) h^{-1} \eta\right)\left(\mathcal{M}_{\chi_{0}}^{T}\left(h \mathbf{d}\left(\Pi_{1}^{-1}\right)\right) \varphi_{0}\right)(-w)\right. \\
& \left.+\sum_{c \in \mathcal{O}_{F} / \pi \mathcal{O}_{F}} \Phi_{0}\left(\Pi_{1}^{k_{1}} \Pi_{2}^{k_{2}} \alpha \mathbf{d}\left(\Pi_{2}^{-1}\right) \mathbf{n}(-c) h^{-1} \eta\right)\left(\mathcal{M}_{\chi_{0}}^{T}\left(h \mathbf{n}(c) \mathbf{d}\left(\Pi_{2}\right)\right) \varphi_{0}\right)(-w)\right\} .
\end{aligned}
$$

(iv) We have

$$
\mathcal{I}_{\Pi_{1}^{k_{1}} \Pi_{2}^{k_{2}} \mathfrak{a}}\left(W * \phi_{0}^{ \pm}, \tau\right)=p^{-k_{1}-k_{2}}|\mathrm{~N}(\alpha)|_{F} \int_{H_{\eta} \backslash H} \int_{K} \chi_{0}^{-1}(\operatorname{det} h) W(h) A_{k_{1}, k_{2}}^{ \pm}(w, h) \overline{\tau\left(w, x_{w}\right)} d w d h,
$$

where

$$
A_{k_{1}, k_{2}}^{ \pm}(w, h)=\chi_{0}\left(\Pi_{1} / \Pi_{2}\right)^{ \pm 1} \Phi_{0}\left(\Pi_{1}^{k_{1}} \Pi_{2}^{k_{2}}\left(\Pi_{1} / \Pi_{2}\right)^{ \pm 1} \alpha h^{-1} \eta\right)\left(\mathcal{M}_{\chi_{0}}^{T}\left(h \mathbf{d}\left(\left(\Pi_{1} / \Pi_{2}\right)^{ \pm 1}\right)\right) \varphi_{0}\right)(-w) .
$$

(v) We have

$$
\begin{aligned}
p \mathcal{I}_{\Pi_{1}^{k_{1}+1} \Pi_{2}^{k_{2} \mathfrak{a}}}(W, \tau)-p^{-1 / 2} \mathcal{I}_{\Pi_{1}^{k_{1}} \Pi_{2}^{k_{2}}}\left(W * \phi_{1}, \tau\right)-\mathcal{I}_{\Pi_{1}^{k_{1}} \Pi_{2}^{k_{2}} \mathfrak{a}}(W, \tau) & +\mathcal{I}_{\Pi_{1}^{k_{1}-1} \Pi_{2}^{k_{2}+1} \mathfrak{a}}\left(W * \phi_{0}^{+}, \tau\right)+p^{-1} \mathcal{I}_{\Pi_{1}^{k_{1}} \Pi_{2}^{k_{2}-1} \mathfrak{a}}(W, \tau) \\
= & -p^{-k_{1}-k_{2}}|\mathrm{~N}(\alpha)|_{F} \int_{H_{\eta} \backslash H} \int_{K} \chi_{0}^{-1}(\operatorname{det} h) W(h) B_{k_{1}, k_{2}}(w, h) \overline{\tau\left(w, x_{w}\right)} d w d h,
\end{aligned}
$$

where

$$
\begin{aligned}
& B_{k_{1}, k_{2}}(w, h) \\
& \quad=p^{-1 / 2} A_{k_{1}, k_{2}}^{1}(w, h)+A_{k_{1}, k_{2}}(w, h)-A_{k_{1}+1, k_{2}}(w, h)-A_{k_{1}, k_{2}-1}(w, h)-A_{k_{1}-1, k_{2}+1}^{+}(w, h) .
\end{aligned}
$$

(vi) We have

$$
\begin{aligned}
& p \mathcal{I}_{\Pi_{1}^{k_{1}} \Pi_{2}^{k_{2}+1} \mathfrak{a}}(W, \tau)-p^{-1 / 2} \mathcal{I}_{\Pi_{1}^{k_{1}} \Pi_{2}^{k_{2} \mathfrak{a}}}\left(W * \phi_{2}, \tau\right)-\mathcal{I}_{\Pi_{1}^{k_{1}} \Pi_{2}^{k_{2} \mathfrak{a}}}(W, \tau) \\
& +\mathcal{I}_{\Pi_{1}^{k_{1}+1} \Pi_{2}^{k_{2}-1} \mathfrak{a}}\left(W * \phi_{0}^{-}, \tau\right)+p^{-1} \mathcal{I}_{\Pi_{1}^{k_{1}-1} \Pi_{2}^{k_{2}} \mathfrak{a}}(W, \tau) \\
& =-p^{-k_{1}-k_{2}}|\mathrm{~N}(\alpha)|_{F} \int_{H_{\eta} \backslash H} \int_{K} \chi_{0}^{-1}(\operatorname{det} h) W(h) B_{k_{1}, k_{2}}^{\prime}(w, h) \overline{\tau\left(w, x_{w}\right)} d w d h \text {, }
\end{aligned}
$$

where

$$
\begin{aligned}
& B_{k_{1}, k_{2}}^{\prime}(w, h) \\
& \quad=p^{-1 / 2} A_{k_{1}, k_{2}}^{2}(w, h)+A_{k_{1}, k_{2}}(w, h)-A_{k_{1}, k_{2}+1}(w, h)-A_{k_{1}-1, k_{2}}(w, h)-A_{k_{1}+1, k_{2}-1}^{-}(w, h) .
\end{aligned}
$$

Proof. The first assertion is obvious from the definition. The assertions (ii)-(iv) follow from Lemma 10.3 and the fact that $\phi_{1}^{\vee}=\phi_{2}, \phi_{2}^{\vee}=\phi_{1}$ and $\left(\phi_{0}^{ \pm}\right)^{\vee}=\phi_{0}^{\mp}$. The last two assertions are direct consequences of assertions (i)-(iv).

By Lemma 11.9, we have

$$
\operatorname{Supp}(W) \subset \bigcup_{l_{1}, l_{2} \in \mathbb{Z}, l_{1}+l_{2} \geqslant 0} H_{\eta} h_{l_{1}, l_{2}} \mathcal{U}
$$

where $h_{l_{1}, l_{2}}=\mathbf{d}\left(\Pi_{1}^{-l_{1}} \Pi_{2}^{-l_{2}} \alpha^{-1}\right)$. Set

$$
\begin{aligned}
\mathcal{B}_{k_{1}, k_{2}, l_{1}, l_{2}} & =\int_{K} B_{k_{1}, k_{2}}\left(w, h_{l_{1}, l_{2}}\right) \overline{\tau\left(w, x_{w}\right)} d w, \\
\mathcal{B}_{k_{1}, k_{2}, l_{1}, l_{2}}^{\prime} & =\int_{K} B_{k_{1}, k_{2}}^{\prime}\left(w, h_{l_{1}, l_{2}}\right) \overline{\tau\left(w, x_{w}\right)} d w .
\end{aligned}
$$

To prove Theorem 10.2, it suffices to show the following. 


\section{A. Murase and T. Sugano}

Proposition 12.5. For $k_{1}, k_{2}, l_{1}, l_{2} \in \mathbb{Z}$ with $k_{1} \geqslant 0, k_{2} \geqslant 0, l_{1}+l_{2} \geqslant 0$, we have $\mathcal{B}_{k_{1}, k_{2}, l_{1}, l_{2}}=0$ and $\mathcal{B}_{k_{1}, k_{2}, l_{1}, l_{2}}^{\prime}=0$.

\subsection{Proof of Proposition 12.5}

To simplify the notation, we set

$$
\begin{gathered}
\phi_{l_{1}, l_{2}}(w)=\varphi_{0}\left(\Pi_{1}^{-l_{1}} \Pi_{2}^{-l_{2}} \alpha^{-1} w\right) \\
J\left(l_{1}, l_{2}\right)=\int_{K} \phi_{l_{1}, l_{2}}(w) \overline{\tau\left(w, x_{w}\right)} d w \\
J^{\prime}\left(l_{1}, l_{2}\right)=\int_{K} \phi_{l_{1}, l_{2}}(w) \psi_{m}(\mathrm{~N}(w)) \overline{\tau\left(w, x_{w}\right)} d w=\int_{K} \phi_{l_{1}, l_{2}}(w) \overline{\tau\left(w,-x_{w}\right)} d w
\end{gathered}
$$

for $l_{1}, l_{2} \in \mathbb{Z}$. Note that $J\left(l_{1}, l_{2}\right)=J^{\prime}\left(l_{1}, l_{2}\right)$ if $l_{1}+l_{2}+\mu \geqslant 0$.

LEMma 12.6. For $k_{1}, k_{2}, l_{1}, l_{2} \in \mathbb{Z}$ with $k_{1} \geqslant 0, k_{2} \geqslant 0, l_{1}+l_{2} \geqslant 0$, we have

$$
\begin{aligned}
\mathcal{B}_{k_{1}, k_{2}, l_{1}, l_{2}=} & p^{\left(l_{1}+l_{2}\right) / 2} \chi_{0}\left(\Pi_{1}^{l_{1}} \Pi_{2}^{l_{2}} \alpha\right)|\mathrm{N}(\alpha)|_{F}^{-1 / 2} \\
& \times \begin{cases}-J\left(l_{1}, l_{2}\right)+p^{-1} J\left(l_{1}-1, l_{2}\right) & \text { if } k_{1}=l_{1}-1, k_{2} \geqslant l_{2}, \\
-J\left(l_{1}-1, l_{2}+1\right)+p^{-1} J^{\prime}\left(l_{1}-1, l_{2}\right) & \text { if } k_{1} \geqslant l_{1}, k_{2}=l_{2}, \\
0 & \text { otherwise }\end{cases}
\end{aligned}
$$

and

$$
\begin{aligned}
\mathcal{B}_{k_{1}, k_{2}, l_{1}, l_{2}}^{\prime}= & p^{\left(l_{1}+l_{2}\right) / 2} \chi_{0}\left(\Pi_{1}^{l_{1}} \Pi_{2}^{l_{2}} \alpha\right)|\mathrm{N}(\alpha)|_{F}^{-1 / 2} \\
& \times \begin{cases}-J\left(l_{1}, l_{2}\right)+p^{-1} J^{\prime}\left(l_{1}, l_{2}-1\right) & \text { if } k_{1} \geqslant l_{1}, k_{2}=l_{2}-1, \\
-J\left(l_{1}+1, l_{2}-1\right)+p^{-1} J\left(l_{1}, l_{2}-1\right) & \text { if } k_{1}=l_{1}, k_{2} \geqslant l_{2}, \\
0 & \text { otherwise. }\end{cases}
\end{aligned}
$$

Proof. By the definitions of $A_{k_{1}, k_{2}}$ and $A_{k_{1}, k_{2}}^{ \pm}$, we have

$$
\begin{gathered}
A_{k_{1}, k_{2}}\left(w, h_{l_{1}, l_{2}}\right)=\delta\left(k_{1} \geqslant l_{1}, k_{2} \geqslant l_{2}\right) p^{\left(l_{1}+l_{2}\right) / 2} \chi_{0}\left(\Pi_{1}^{l_{1}} \Pi_{2}^{l_{2}} \alpha\right)|\mathrm{N}(\alpha)|_{F}^{-1 / 2} \phi_{l_{1}, l_{2}}(w), \\
A_{k_{1}, k_{2}}^{+}\left(w, h_{l_{1}, l_{2}}\right)=\delta\left(k_{1} \geqslant l_{1}-1, k_{2} \geqslant l_{2}+1\right) p^{\left(l_{1}+l_{2}\right) / 2} \chi_{0}\left(\Pi_{1}^{l_{1}} \Pi_{2}^{l_{2}} \alpha\right)|\mathrm{N}(\alpha)|_{F}^{-1 / 2} \phi_{l_{1}-1, l_{2}+1}(w), \\
A_{k_{1}, k_{2}}^{-}\left(w, h_{l_{1}, l_{2}}\right)=\delta\left(k_{1} \geqslant l_{1}+1, k_{2} \geqslant l_{2}-1\right) p^{\left(l_{1}+l_{2}\right) / 2} \chi_{0}\left(\Pi_{1}^{l_{1}} \Pi_{2}^{l_{2}} \alpha\right)|\mathrm{N}(\alpha)|_{F}^{-1 / 2} \phi_{l_{1}+1, l_{2}-1}(w)
\end{gathered}
$$

for $k_{1}, k_{2}, l_{1}, l_{2} \in \mathbb{Z}$. Next suppose that $k_{1}, k_{2}, l_{1}+l_{2} \geqslant 0$. By using the formula

$$
\sum_{c \in \mathcal{O}_{F} / \pi \mathcal{O}_{F}} \mathcal{M}_{\chi_{0}}^{T}\left(\mathbf{n}\left(\pi^{-1} c\right)\right) \varphi_{0}(w)=p\left\{\varphi_{0}\left(\Pi_{1}^{-1} w\right)+\varphi_{0}\left(\Pi_{2}^{-1} w\right)-\varphi_{0}\left(\pi^{-1} w\right)\right\},
$$

we obtain

$$
\begin{aligned}
& A_{k_{1}, k_{2}}^{1}\left(w, h_{l_{1}, l_{2}}\right) \\
& \quad=p^{\left(l_{1}+l_{2}+1\right) / 2} \chi_{0}\left(\Pi_{1}^{l_{1}} \Pi_{2}^{l_{2}} \alpha\right)|\mathrm{N}(\alpha)|_{F}^{-1 / 2} \times\left[\delta\left(k_{1} \geqslant l_{1}, k_{2} \geqslant l_{2}+1\right)\left\{\phi_{l_{1}, l_{2}}(w)+\phi_{l_{1}-1, l_{2}+1}(w)\right\}\right. \\
& \left.\quad+p^{-1} \delta\left(k_{1}=l_{1}-1, k_{2} \geqslant l_{2}\right) \phi_{l_{1}-1, l_{2}}(w)+p^{-1} \delta\left(k_{1} \geqslant l_{1}, k_{2}=l_{2}\right) \psi_{m}(\mathrm{~N}(w)) \phi_{l_{1}-1, l_{2}}(w)\right]
\end{aligned}
$$

and

$$
\begin{aligned}
& A_{k_{1}, k_{2}}^{2}\left(w, h_{l_{1}, l_{2}}\right) \\
& \quad=p^{\left(l_{1}+l_{2}+1\right) / 2} \chi_{0}\left(\Pi_{1}^{l_{1}} \Pi_{2}^{l_{2}} \alpha\right)|\mathrm{N}(\alpha)|_{F}^{-1 / 2} \times\left[\delta\left(k_{1} \geqslant l_{1}+1, k_{2} \geqslant l_{2}\right)\left\{\phi_{l_{1}, l_{2}}(w)+\phi_{l_{1}+1, l_{2}-1}(w)\right\}\right. \\
& \left.\quad+p^{-1} \delta\left(k_{1}=l_{1}, k_{2} \geqslant l_{2}-1\right) \phi_{l_{1}, l_{2}-1}(w)+p^{-1} \delta\left(k_{1} \geqslant l_{1}+1, k_{2}=l_{2}-1\right) \psi_{m}(\mathrm{~N}(w)) \phi_{l_{1}, l_{2}-1}(w)\right] .
\end{aligned}
$$




\section{ON THE Fourier-JACOBI EXPANSION OF THE UNITARY KUDLA LIFT}

A straightforward calculation shows that

$$
\begin{aligned}
B_{k_{1}, k_{2}}\left(w, h_{l_{1}, l_{2}}\right)= & p^{\left(l_{1}+l_{2}\right) / 2} \chi_{0}\left(\Pi_{1}^{l_{1}} \Pi_{2}^{l_{2}} \alpha\right)|\mathrm{N}(\alpha)|^{-1 / 2} \\
& \times\left[\delta\left(k_{1}=l_{1}-1, k_{2} \geqslant l_{2}\right)\left\{-\phi_{l_{1}, l_{2}}(w)+p^{-1} \phi_{l_{1}-1, l_{2}}(w)\right\}\right. \\
& \left.+\delta\left(k_{1} \geqslant l_{1}, k_{2}=l_{2}\right)\left\{-\phi_{l_{1}-1, l_{2}+1}(w)+p^{-1} \psi_{m}(\mathrm{~N}(w)) \phi_{l_{1}-1, l_{2}}(w)\right\}\right]
\end{aligned}
$$

and

$$
\begin{aligned}
B_{k_{1}, k_{2}}^{\prime}\left(w, h_{l_{1}, l_{2}}\right)= & p^{\left(l_{1}+l_{2}\right) / 2} \chi_{0}\left(\Pi_{1}^{l_{1}} \Pi_{2}^{l_{2}} \alpha\right)|\mathrm{N}(\alpha)|^{-1 / 2} \\
& \times\left[\delta\left(k_{1} \geqslant l_{1}, k_{2}=l_{2}-1\right)\left\{-\phi_{l_{1}, l_{2}}(w)+p^{-1} \psi_{m}(\mathrm{~N}(w)) \phi_{l_{1}, l_{2}-1}(w)\right\}\right. \\
& \left.+\delta\left(k_{1}=l_{1}, k_{2} \geqslant l_{2}\right)\left\{-\phi_{l_{1}+1, l_{2}-1}(w)+p^{-1} \phi_{l_{1}, l_{2}-1}(w)\right\}\right] .
\end{aligned}
$$

These immediately show the lemma.

To complete the proof of Proposition 12.5, it is sufficient to show the following.

Lemma 12.7. Let $l_{1}, l_{2} \in \mathbb{Z}$ with $l_{1}+l_{2} \geqslant 0$.

(i) If $l_{1} \geqslant 1$, we have $J\left(l_{1}, l_{2}\right)=p^{-1} J\left(l_{1}-1, l_{2}\right)$.

(ii) If $l_{2} \geqslant 1$, we have $J\left(l_{1}, l_{2}\right)=p^{-1} J^{\prime}\left(l_{1}, l_{2}-1\right)$.

Proof. Suppose that $l_{1}+l_{2} \geqslant 0$ and $l_{2} \geqslant 1$. Take $\alpha=\left(\alpha_{1}, \alpha_{2}\right) \in K^{\times}$such that $\mathfrak{a}=\alpha \mathcal{O}_{K}$. We then have

$$
\begin{aligned}
J\left(l_{1}, l_{2}\right) & =\int_{\Pi_{1}^{l_{1}} \Pi_{2}^{l_{2} \mathfrak{a}}} \overline{\tau\left(w, x_{w}\right)} d w \\
& =\left|\pi^{l_{2}-1} \alpha_{2}\right|_{F}^{-1} \int_{\Pi_{1}^{l_{1}} \Pi_{2}^{l_{2} \mathfrak{a}}} \int_{\pi^{l_{2}-1} \alpha_{2} \mathcal{O}_{F}} \overline{\frac{\tau\left(\left(w, x_{w}\right)((0, u), 0)\right)}{\tau\left(w+(0, u), \frac{1}{2} w_{1} w_{2}-\frac{1}{2} w_{1} u\right)}} d u d w \\
& =\left|\pi^{l_{2}-1} \alpha_{2}\right|_{F}^{-1} \int_{\Pi_{1}^{l_{1}} \Pi_{2}^{l_{2}} \mathfrak{a}} \int_{\pi^{l_{2}-1} \alpha_{2} \mathcal{O}_{F}} \bar{\tau} \overline{\left.\tau\left(w_{1}, w_{2}+u\right),-\frac{1}{2} w_{1}\left(w_{2}+u\right)\right)} d u d w \\
& =\left|\pi^{l_{2}-1} \alpha_{2}\right|_{F}^{-1} \int_{\Pi_{1}^{l_{1}} \Pi_{2}^{l_{2}} \mathfrak{a}} \int_{\pi^{l_{2}-1} \alpha_{2} \mathcal{O}_{F}} \overline{\tau\left(w,-x_{w}\right)} d w \\
& =p^{-1} \int_{\Pi_{1}^{l_{1}} \Pi_{2}^{l_{2}-1} \mathfrak{a}} \overline{\tau(w),} \\
& =p^{-1} J^{\prime}\left(l_{1}, l_{2}-1\right),
\end{aligned}
$$

which proves assertion (ii). The proof of assertion (i) is similar and we omit it.

\section{ACKNowledgements}

The authors would like to thank the referee for his careful reading of the manuscript and for valuable suggestions and comments.

\section{REFERENCES}

BPR03 E. M. Baruch, I. Piatetski-Shapiro and S. Rallis, On the uniqueness of Fourier Jacobi models for representations of $\mathrm{U}(2,1)$, in Lie groups and symmetric spaces, American Mathematical Society Translations, Series 2, vol. 210 (American Mathematical Society, Providence, RI, 2003), 47-56.

GR89 G. I. Glauberman and J. D. Rogawski, On theta functions with complex multiplication, J. reine angew. Math. 395 (1989), 68-101. 


\section{ON THE FOURIER-JACOBI EXPANSION OF THE UNITARY KUDLA LIFT}

GRS97 S. Gelbart, J. D. Rogawski and D. Soudry, Endoscopy, theta liftings, and period integrals for the unitary group in the three variables, Ann. of Math. (2) 145 (1997), 419-476.

HKS96 M. Harris, S. S. Kudla and W. J. Sweet, Theta dichotomy for unitary groups, J. Amer. Math. Soc. 9 (1996), 941-1004.

Kud79 S. Kudla, On certain arithmetic automorphic forms for $\mathrm{SU}(1, q)$, Invent. Math. 52 (1979), 1-25.

Kud81 S. Kudla, On certain Euler products for SU(2,1), Compositio Math. 42 (1981), 321-344.

Li75 W.-C. W. Li, Newforms and functional equations, Math. Ann. 212 (1975), 285-315.

MVW87 C. Mœglin, M.-F. Vignéras and J.-L. Waldspurger, Correspondences de Howe sur un corps p-adique, Lecture Notes in Mathematics, vol. 1291 (Springer, New York, 1987).

Moe91 C. Moen, The dual pair $(U(1), U(1))$ over a p-adic field, Pacific J. Math. 158 (1991), 365-386.

Mur01 A. Murase, On metaplectic representations of unitary groups: I. Splitting, Proc. Japan Acad. Ser. A Math. Sci. 77 (2001), 59-62.

MS00 A. Murase and T. Sugano, Local theory of primitive theta functions, Compositio Math. 123 (2000), 273-302.

MS02 A. Murase and T. Sugano, Fourier-Jacobi expansion of Eisenstein series on unitary groups of degree three, J. Math. Sci. Univ. Tokyo 9 (2002), 347-404.

MS06 A. Murase and T. Sugano, Inner product formula for Kudla lift, in Automorphic forms and zeta functions, in memory of Tsuneo Arakawa (World Scientific, Singapore, 2006), 280-313.

Pia80 I. I. Piatetski-Shapiro, Tate theory for reductive groups and distinguished representations, in Proc. Int. Cong. Math., Helsinki, 1978 (Academia Scientiarum Fennica, Helsinki, 1980), 585-590.

Rog92 J. D. Rogawski, The multiplicity formula for A-packets, in The zeta functions of Picard modular surfaces, eds R. P. Langlands and D. Ramakrishnan (Les Publications CRM, Montréal, 1992), 395-419.

Shi79 T. Shintani, On automorphic forms on unitary groups of order 3, Unpublished manuscript (1979).

Tan99 V. Tan, An application of the regularized Siegel-Weil formula on unitary groups to a theta lifting problem, Proc. Amer. Math. Soc. 127 (1999), 2811-2820.

Tat79 J. Tate, Number theoretic background, Proceedings of Symposia in Pure Mathematics, vol. 33 (American Mathematical Society, Providence, RI, 1979), 3-26.

Wei64 A. Weil, Sur certains groupes d'opérateurs unitaires, Acta Math. 111 (1964), 143-211.

Yan97 T. Yang, Theta liftings and Hecke L-functions, J. reine angew. Math. 485 (1997), 25-53.

Atsushi Murase murase@cc.kyoto-su.ac.jp

Department of Mathematical Science, Faculty of Science, Kyoto Sangyo University, Motoyama, Kamigamo, Kita-ku, Kyoto 603-8555, Japan

Takashi Sugano sugano@kappa.s.kanazawa-u.ac.jp

Department of Mathematics, Faculty of Science, Kanazawa University, Kakuma-machi, Kanazawa 920-1192, Japan 